Geometría y dinámica en el problema de $\mathrm{N}$ vórtices puntuales.
Con base en la Legislación de la Universidad Autónoma Metropolitana, en la Ciudad de MEXICO, se presentaron a las 11:00 horas del día 28 del mes de junio del año 2016, los suscritos miembros del jurado designado por la Comisión del Posgrado:

DR. RAMON PLAZA VILLEGAS

DR. MARTIN CELLI

DR. ANTONIO HERNANDEZ GARDUÑO
Bajo la Presidencia del primero y con carácter de Secretario el último, se reunieron para proceder al Examen de Grado cuya denominación aparece al margen, para la obtención del grado de:

MAESTRO EN CIENCIAS (MATEMÁTICAS)

DE： JOSE LUIS QUIROZ HERRERA

$y$ de acuerdo con el artículo 78 fracción III del Reglamento de Estudios Superiores de la Universidad Autónoma Metropolitana, los miembros del jurado resolvieron:

\section{Aprobar}

Acto continuo, el presidente del jurado comunicó al interesado el resultado de la evaluación $y$, en caso aprobatorio, le fue tomada la protesta.

LIC. JULIO CESAR QE LARA ISASSI DIRECTOR DE SISTEMAS ESQQOLARES

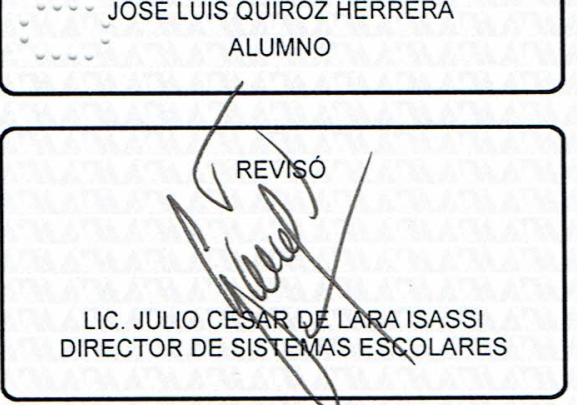

DIRECTOR DE LA DIVISIÓN DE CBI

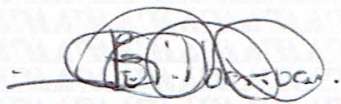

DR. JOSE GILBERTO CORDOBA HERRERA
PRESIDENTE

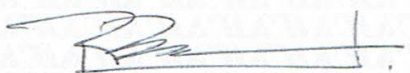

DR. RAMON PLAZA VILLEGAS

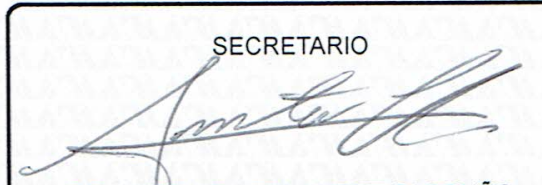

DR. ANTONIO HERNANDEZ GARDUÑO 


\title{
Geometría y dinámica en el problema de $N$ vórtices puntuales
}

\author{
José Luis Quiroz Herrera \\ Asesor: Dr. Antonio Hernández Garduño
}

4 de junio del 2016 


\section{Resumen}

En este trabajo estudiamos el problema de $N$ vórtices puntuales, estableciendo las ecuaciones de movimiento y su estructura Hamiltoniana. Nos concentramos luego en el problema de tres vórtices puntuales y discutimos su reducción simpléctica. Hay una estructura geométrica y estructura de Poisson asociadas con esta reducción. El principal objetivo de este trabajo es clarificar dichas estructuras. 


\section{Agradecimientos}

Quiero agradecer a mis Padres por el apoyo que me brindaron durante este proceso. A mi esposa por su comprensión y apoyo. Mi asesor por su enorme paciencia, comprensión y su gran apoyo para que lograra concluir el presente trabajo. Les agradezco a todos por su gran apoyo. 


\section{Índice general}

1. Introducción 3

2. Preliminares 14

2.1. Variedades . . . . . . . . . . . . . . . . . . . . 14

2.1.1. Campos vectoriales. . . . . . . . . . . . . . 15

2.2. Variedades simplécticas y de Poisson . . . . . . . . . . 17

2.2.1. Espacios vectoriales simplécticos y de Poisson . . . . . 17

2.2.2. Grupos y álgebras de Lie . . . . . . . . . . . . . . . 19

2.2.3. El álgebra de Lie asociada a un grupo de Lie . . . . . . 21

2.2.4. El grupo $U(2)$ y su álgebra $\mathfrak{u}(2) \ldots \ldots \ldots \ldots$. . . . . 22

2.2.5. El dual de una álgebra de Lie . . . . . . . . . . . . . 24

2.2.6. Orbitas coadjuntas y foliación simpléctica . . . . . . . 24

2.2.7. Orbitas coadjuntas en $\mathfrak{u}(2)^{*} \ldots \ldots \ldots . . \ldots 26$

2.3. Dinámica hamiltoniana . . . . . . . . . . . . . . . . . . . 27

2.3.1. Formulación Canónica . . . . . . . . . . . . . 28

2.3.2. $\quad$ Braquet de Poisson . . . . . . . . . . . . . . . . . . . 29

2.3.3. Formulación no canónica . . . . . . . . . . . . . . . 32

2.4. Función $\delta$ de Dirac . . . . . . . . . . . . . . . . . . . . 33

3. Dinámica de vorticidad 36

3.1. Nociones básicas . . . . . . . . . . . . . . . . 36

3.2. Invariantes de las ecuaciones de Euler . . . . . . . . . . . . 42

3.3. Vorticidad en dos dimensiones . . . . . . . . . . . . . . . . . 45

3.4. Descomposición en vórtices puntuales . . . . . . . . . . . . . 51

3.5. Otras dinámicas de vorticidad en el plano . . . . . . . . . . 53

3.6. Integración del problema de tres vórtices . . . . . . . . . 55 
4. El problema de $N$ vórtices en el plano 58

4.1. Formulación general . . . . . . . . . . . . . . . 58

4.1.1. Teorema de Synge . . . . . . . . . . . . . . . . . 61

4.2. Casos especiales . . . . . . . . . . . . . . . . . . . . 62

4.2.1. El problema restringido de $N$-vórtices . . . . . . . . . 62

4.2.2. Soluciones autosimilares $\ldots \ldots \ldots$. . . . . . . . 63

5. El problema de tres vórtices en el plano 67

5.1. El problema restringido de tres vórtices . . . . . . . . . . . 67

5.2. Ecuaciones de movimiento usando el cuadrado de las distancias 70

5.3. El cono de las formas . . . . . . . . . . . . . . . . . . 71

\begin{tabular}{|lll|}
\hline 6. & Álgebra y reducción del problema de tres vórtices & 74
\end{tabular}

6.1. Inmersión del cono de las formas en $\mathbb{R}^{4} \ldots \ldots$. . . . . . 74

6.1.1. Reducción de Poisson . . . . . . . . . . . . . . . . 75

6.2. Isomorfismo entre $\mathcal{V}$ y $\mathfrak{u}(2)^{*} \ldots \ldots \ldots \ldots \ldots 78$

6.3. Cantidades conservadas y Casimires . . . . . . . . . . . . 85

6.4. Reducción del problema de tres vórtices como una órbita coad-

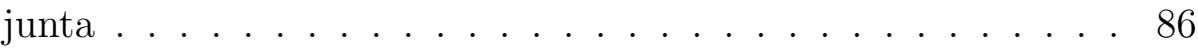

6.4.1. Superficies de nivel de las primeras integrales. . . . . . 89

6.4.2. Estructura simpléctica e interpretación . . . . . . . . 92

6.4.3. Colisiones binarias. . . . . . . . . . . . . . 96

$\begin{array}{ll}\text { 7. Conclusiones } & 99\end{array}$ 


\section{Capítulo 1}

\section{Introducción}

En nuestra vida diaria percibimos, consciente ó inconscientemente, muchos fenómenos descritos por la Física. Uno de ellos es el movimiento, el cual es algo natural para nosotros: se manifiesta simplemente en nuestro desplazamiento de un punto a otro en un tiempo determinado. El movimiento, definido por la Física, considera por ejemplo la evolución de una partícula o de un sistema de partículas en el tiempo. En este sentido, la Mecánica Clásica se enmarca en una teoría más general denominada Sistemas Dinámicos.

\section{Movimiento, fluidos y vórtices}

Una forma en que experimentamos movimiento en la vida cotidiana es cuando observamos ó interactuamos con fluidos (gases y líquidos): vemos correr el agua de un río, vertimos una cucharada de miel sobre nuestro pan en el desayuno, sentimos nuestra respiración y el pulso de la sangre en nuestras venas, transpiramos gotas de sudor después de una actividad extenuante, y saciamos nuestra sed con agua ó cerveza. Los fluidos manifiestan una variedad de fenómenos interesantes, como la tensión superficial que permite a los mosquitos caminar sobre el agua, la transmisión del sonido y ondas de choque, ó la formación de remolinos, como en los tornados y huracanes. Quizá el ejemplo más dramático de éstos últimos es la gran mancha roja de Júpiter, un huracán que ha permanecido activo por más de cuatrocientos años.

Podemos nosotros mismo generar remolinos, un fenómeno muy llamativo a la vista, cuando por ejemplo agitamos nuestra taza de café para mezclar el líquido, ó al drenar una tina llena de agua. Estos remolinos se conocen como 

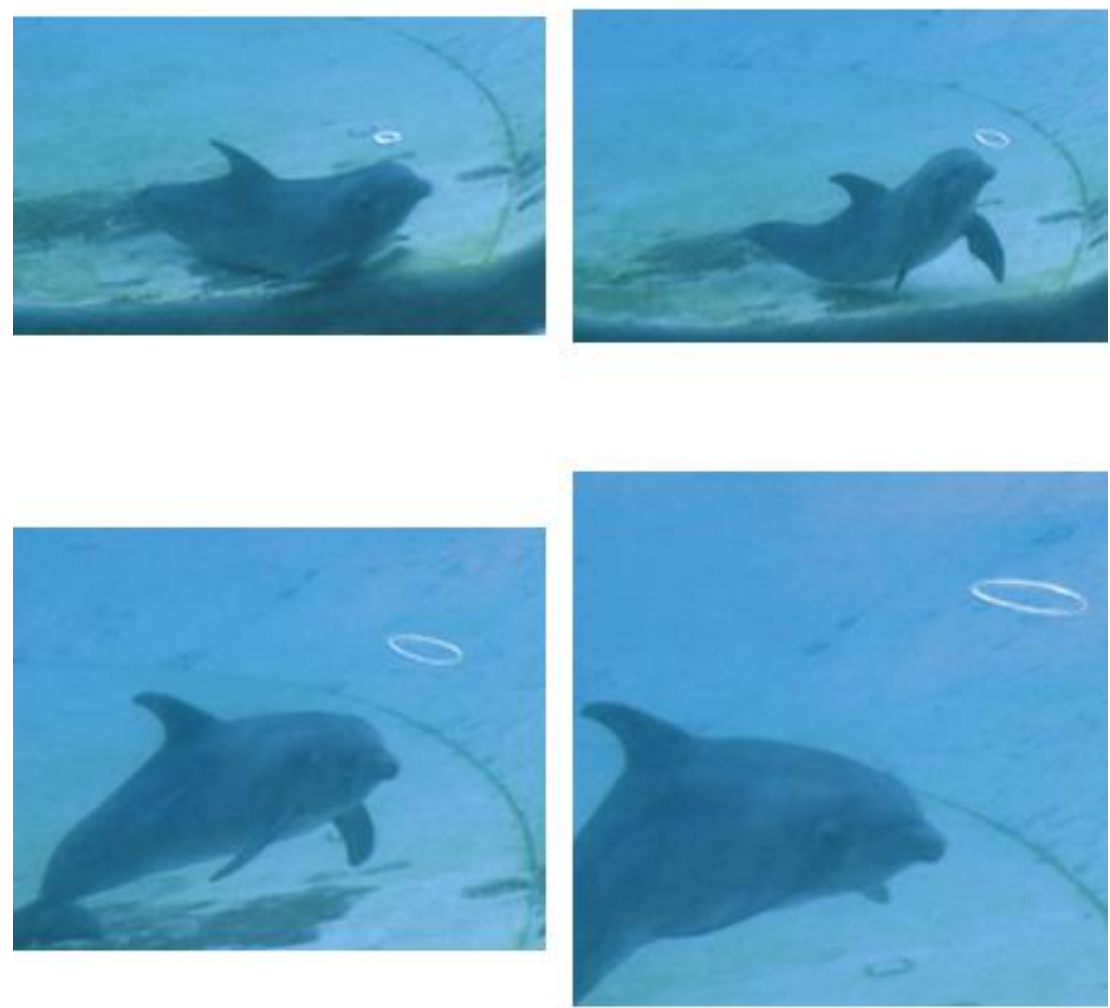

Figura 1.1: Secuencia mostrando un delfín jugando con un anillo de vorticidad. Tomado de [25].

vórtices. Algunos fumadores se entretienen haciendo aros de humo circulares; éstos son anillos de vorticidad. Un ejemplo más atractivo aún son los anillos de vorticidad con los que juegan los delfines, como el que se muestra en la figura 1.1 .

Matemáticamente, la vorticidad es el rotacional del campo de velocidad de un fluido. Para darnos una idea intuitiva de su significado, imaginemos una curva cerrada acotando una pequeña región donde la vorticidad es casi constante. Como la circulación de la velocidad a lo largo de la curva es proporcional a la magnitud de la vorticidad (teorema de Stokes), podemos intuir a la vorticidad como una medida del giro que experimentaría un pequeño ob- 
jeto arrojado sobre dicha regioncita. Cuando la vorticidad está concentrada en una región tubular delgada, se tiene un filamento de vorticidad. Si el filamento forma una curva cerrada simple, tenemos un anillo de vorticidad, como los que la figura 1.1 ilustra.

Podemos simplificar la descripción matemática de un fluido ignorando su viscosidad. Esto nos lleva al concepto de fluidos ideales, los cuales están gobernados por las ecuaciones de Euler. En \$3.1, ecuación (3.14), damos las ecuaciones de Euler para fluidos incompresibles. Al restringir nuestro estudio a fluidos ideales perdemos, desde luego, la capacidad de describir algunos fenómenos interesantes, pero aún así somos capaces de entender otros más como la dinámica de vorticidad. De hecho, las ecuaciones de Euler (3.14) se pueden escribir en términos del campo de vorticidad, como lo muestra la ecuación (3.15).

Se sabe que un anillo de vorticidad se autoinduce una velocidad que es proporcional a su curvatura (cf. [30, §8.1]). En contraste, los filamentos rectos no se autoinducen velocidad. De esta manera, mientras que un anillo aislado tiene movimiento, un solo filamento rectilíneo permanecería en reposo. Ésta es la principal diferencia entre los anillos y los filamentos rectos de vorticidad.

En este trabajo, consideraremos vórtices puntuales, los cuales se interpretan como sigue. En un sistema de filamentos de vorticidad rectilíneos y paralelos, imaginamos que hacemos un corte con un plano ortogonal a los filamentos. Cada filamento de vorticidad queda caracterizado por la proyección de su eje central sobre este plano y la intensidad de su vorticidad. Un vórtice puntual queda así representado por un par de datos: su posición en el plano bajo esta proyección y la medida de su vorticidad. De esta manera, el sistema de filamentos de vorticidad queda descrito por un conjunto de vórtices puntuales sobre el plano. El modelo de interacción de estos vórtices es descrito por las ecuaciones (3.24).

Estas ecuaciones son Hamiltonianas; a grandes rasgos, esto quiere decir que las ecuaciones tienen la estructura de las ecuaciones de movimiento de la mecánica clásica cuando se sigue el formalismo introducido por Hamilton en 1833. (Una diferencia importante es que, en contraste con los sistemas de partículas de mecánica clásica, en el problema de $N$ vórtices el espacio fase coincide con el espacio de configuración.) 


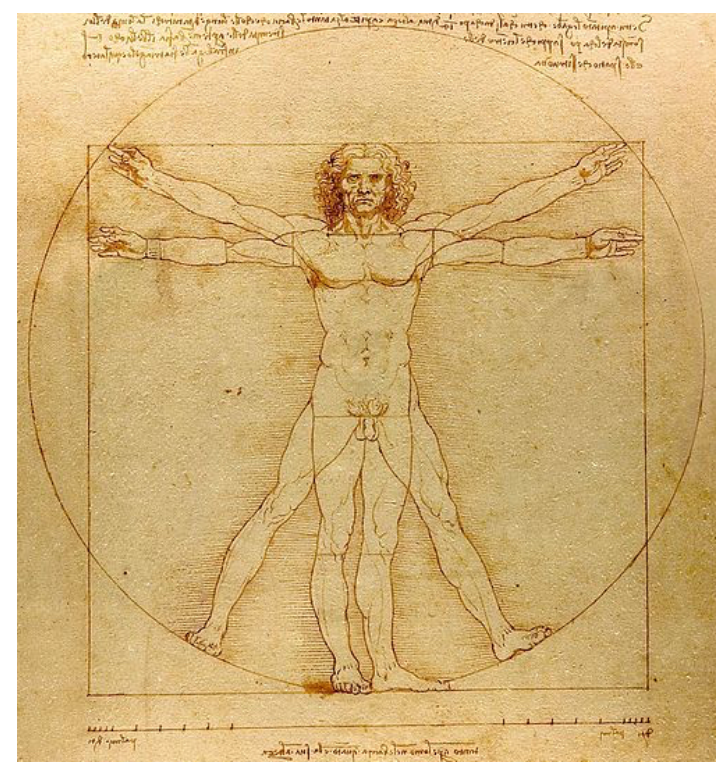

Figura 1.2: "El Hombre de Vitruvio", una obra de Leonardo Da Vinci, ilustra el concepto de simetría de reflexión y un primer ejemplo de la noción de grupo. Tomado de [41].

\section{Simetría}

Al observar a nuestro alrededor, apreciamos cómo la simetría aparece en muchas instancias de nuestra experiencia cotidiana. Recordando la obra de Leonardo da Vinci El Hombre de Vitruvio, figura 1.2, ó bien al observarnos en el espejo, tomamos consciencia de que la simetría se manifiesta en nuestro propio cuerpo, toda vez que los rasgos y proporciones se mantienen después de una reflexión con respecto al eje vertical. En lenguaje matemático, decimos que la imagen de nuestro cuerpo es invariante con respecto a reflexiones con respecto a dicho eje. Las artes plásticas y la arquitectura están llenas de ejemplos de simetría.

En matemáticas el lenguaje apropiado para describir la simetría, se basa en la noción de grupo. Podemos decir que la simetría es la invariancia de alguna característica después de alguna transformación. Uno de los teoremas más celebrados en Mecánica Clásica es el Teorema de Noether, el cual establece una relación entre cantidades conservadas y las simetrías de las ecuaciones de movimiento. Así por ejemplo, si las ecuaciones de movimiento 
de un sistema mecánico son invariantes bajo la acción del grupo de rotaciones $S O(3)$ entonces el Momento Angular es una cantidad conservada. $S O(3)$ es un ejemplo de grupo de Lie. En general, la cantidad conservada asociada a un grupo de simetría $G$ se llama función de momento (generalmente denotado $J)$.

La noción de grupo de Lie juega un papel muy importante en física y mecánica. A grandes rasgos, un grupo de Lie es un grupo que también es una variedad, y tal que sus operaciones algebraicas son compatibles con su estructura diferenciable. Cuando las ecuaciones de movimiento de un sistema mecánico son invariantes bajo la acción de un grupo de Lie es posible simplificar la descripción de la dinámica usando la noción de sistema reducido.

A grandes rasgos, el proceso de reducción consiste en a) restringirse a un conjunto de nivel $J^{-1}(\mu)$ de la función de momento y b) tomar el cociente con respecto al subgrupo $G_{\mu}$ - del grupo de simetría - que deja fijo el valor $\mu$ de la función de momento. Dicho cociente generalmente se denota $P_{\mu}:=J^{-1}(\mu) / G_{\mu}$. En los casos más simples, la codimensión de $J^{-1}(\mu)$ es igual a $\operatorname{dim} G_{\mu}$. Adicionalmente, tomar el cociente reduce la dimensión de $J^{-1}(\mu)$ otra vez por $\operatorname{dim} G_{\mu}$. Así, pasar del espacio fase original $P$ al espacio reducido $P_{\mu}$ reduce la dimensión del problema en $2 \operatorname{dim} G_{\mu}$.

Para entenderlo mejor, consideremos a continuación dos ejemplos que ilustran el concepto de reducción.

\section{Ejemplo: el problema de Kepler}

El problema de Kepler, que consiste en dos masas puntuales cuya interacción es gobernada por el potencial Newtoniano, se puede simplificar (reducir) usando la Ley de Conservación del Momento Angular (el momento angular respecto del centro de atracción se conserva). Utilizando coordenadas polares escribimos la energía total en términos de la distancia $r$ entre el centro de atracción (sol) y el segundo cuerpo (planeta), y un ángulo medido a partir de una línea de referencia elegida adecuadamente. Así,

$$
E=\frac{1}{2} m\left(\dot{r}^{2}+r^{2} \dot{\theta}^{2}\right)+U(r)
$$

donde, en unidades apropiadas, $U(r)=-m / r$. Aprovechamos ahora la conservación del momento angular $L=m r^{2} \dot{\theta}$ para poder ignorar la velocidad angular. Así, sustituyendo

$$
r \dot{\theta}=\frac{L}{m r} .
$$


en la ecuación (1.1) obtenemos

$$
E=\frac{1}{2} m \dot{r}^{2}+\frac{L^{2}}{2 m r^{2}}+U(r)
$$

Con esto logramos escribir la energía total en términos de $r$ y $\dot{r}$. Derivando con respecto al tiempo y usando que la energía total es constante, obtenemos

$$
m \ddot{r}=-\frac{d V_{\mathrm{eff}}}{d r}
$$

donde el potencial efectivo queda definido como

$$
V_{\mathrm{eff}}:=\frac{L^{2}}{2 m} \frac{1}{r^{2}}+U(r)
$$

De esta manera se logra reducir la descripción de la dinámica del problema de Kepler a la ecuación de movimiento que corresponde a un sistema mecánico con un grado de libertad.

Esta dinámica reducida del problema de Kepler nos da información útil sobre cómo se mueve el planeta, describiendo su movimiento en un marco de referencia rotatorio. (Adicionalmente, sustituyendo $r(t)$ en las ecuaciones de movimiento originales, podemos reconstruir también la dinámica angular que ha sido ignorada en la dinámica reducida, obteniendo así una descripción completa del sistema.)

\section{Ejemplo: el cuerpo rígido libre}

Otro ejemplo de reducción, incluso más relevante para el tema de esta tesis, es el del cuerpo rígido libre. Éste consiste en un sistema mecánico con espacio de configuración $Q=S O(3)$ (rotaciones en el espacio) y energía invariante con respecto a rotaciones. Intuitivamente, esto corresponde a un cuerpo rígido moviéndose en ausencia de fuerzas externas. Notemos que el grupo de simetría coincide en este caso con el espacio de configuración:

$$
G=S O(3)=Q \text {. }
$$

El espacio fase es $T^{*} Q=T^{*} S O(3)$, el cual identificamos con el producto del grupo y el dual de su álgebra de Lie,

$$
T^{*} S O(3) \cong S O(3) \times \mathfrak{s o}(3)^{*} .
$$


Notemos también que podemos identificar $\mathfrak{s o}(3)^{*}$ con $\mathbb{R}^{3}$.

Un estado del sistema queda parametrizado por $(R, \boldsymbol{\Pi}) \in S O(3) \times \mathfrak{s o}(3)^{*}$. Como suponemos que la energía es invariante bajo rotaciones, el hamiltoniano solo depende de $\Pi$. De hecho, éste viene dado por:

$$
H(\boldsymbol{\Pi})=\frac{1}{2}\left(\frac{\Pi_{1}^{2}}{I_{1}}+\frac{\Pi_{2}^{2}}{I_{2}}+\frac{\Pi_{3}^{2}}{I_{3}}\right)
$$

donde $I_{1}, I_{2}, I_{3}$ son los momentos principales de inercia. El vector $\Pi \in \mathbb{R}^{3} \cong$ $\mathfrak{s o}(3)^{*}$ representa el momento angular visto desde un marco de referencia anclado al cuerpo.

El dual de toda álgebra de Lie posee una estructura de Poisson. En el caso concreto de $\mathfrak{s o}(3)^{*} \cong \mathbb{R}^{3}$, dicha estructura está dada por el braquet de Lie-Poisson

$$
\{F, H\}(\boldsymbol{\Pi})=-\boldsymbol{\Pi} \cdot(\nabla F(\boldsymbol{\Pi}) \times \nabla H(\boldsymbol{\Pi})) .
$$

Con este braquet, el hamiltoniano 1.2 induce ecuaciones de movimiento dadas por la ecuación de Hamilton, que para un espacio de Poisson general está dada por la ecuación (2.20), y que para el cuerpo rígido se traducen en la ecuación

$$
\dot{\Pi}=\Pi \times I^{-1} \Pi
$$

donde $I$ es la matriz de momentos de inercia del cuerpo rígido. (En el ejemplo 2.3.5 de la sección 2.3.3 exploramos las ecuaciones del cuerpo rígido con más detalle.)

De esta manera, la descripción de la dinámica del sistema se ha reducido de un sistema de ecuaciones diferenciales de primer orden en seis variables (correspondientes al espacio fase $T^{*} S O(3)$ ) a un sistema de ecuaciones de primer orden en tres variables. El nuevo espacio fase en donde ahora miramos la dinámica es $\mathfrak{s o}(3)^{*} \cong \mathbb{R}^{3}$. Físicamente esto corresponde a describir la dinámica dando la posición del vector de momento angular desde el marco de referencia del cuerpo rígido.

Más aún, de (1.3) se observa que $\dot{\Pi}$ es perpendicular a $\Pi$. En consecuencia, la dinámica reducida tiene lugar sobre esferas centradas en el origen; véase la figura 1.3. Éstas son las hojas simplécticas que folían a $\mathbb{R}^{3}$. Esto ilustra un hecho general: toda variedad de Poisson está foliada por subvariedades simplécticas que son invariantes de la dinámica inducida por un hamiltoniano. (Las nociones de estructura y variedades de Poisson se discuten en la sección 2.2.) 


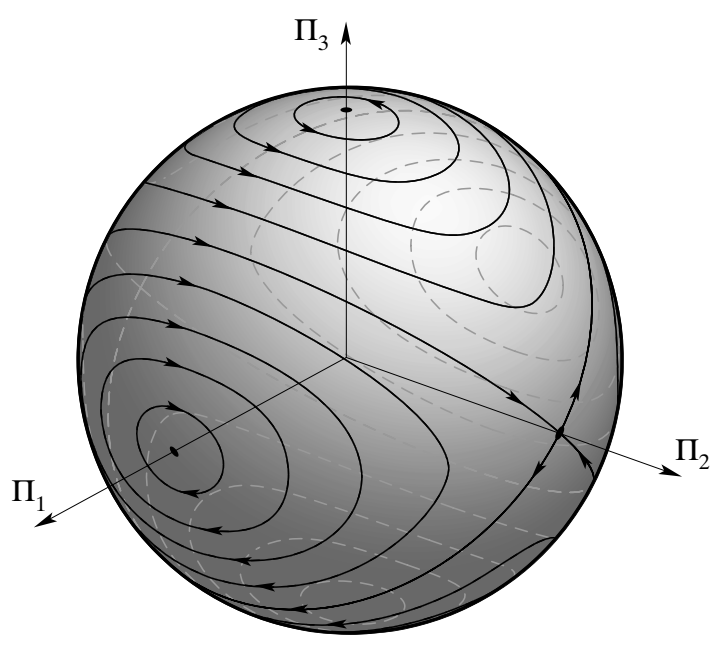

Figura 1.3: Flujo de la dinámica reducida del cuerpo rígido sobre una esfera de momento angular constante (órbita coadjunta), para el caso $I_{1}<I_{2}<I_{3}$. Éste se obtiene intersecando la esfera de momento angular constante con los elipsoides de energía constante. (Tomado de 24, cap. 15].)

\section{Reducción de Lie-Poisson y el problema de tres vórtices}

La reducción que se observa en el cuerpo rígido libre es un ejemplo de un tipo de reducción más general conocida como reducción de Lie-Poisson. Sea $G$ un grupo de Lie. La reducción de Lie-Poisson consiste en comenzar con un sistema hamiltoniano definido sobre $T^{*} G$, tal que el grupo de simetría coincide con $G$, y terminando con un sistema hamiltoniano reducido con espacio fase $\mathfrak{g}^{*}$. Siempre que el espacio fase es el haz cotangente de un grupo de Lie, y el grupo de simetría del hamiltoniano coincide con dicho grupo de Lie, la reducción de Lie-Poisson se puede implementar.

Consideremos ahora el problema de $N$ vórtices puntuales. En la sección 4.1 damos las ecuaciones de movimiento, Hamiltoniano y estructura simpléctica para este sistema. Su grupo de simetría es $G=S E(2)$, el grupo Euclidiano. Una posible estrategia para reducir el problema de $N$-vórtices es intentar adaptar el método de reducción simpléctica descrito arriba (ó más precisamente, el método de reducción simpléctica de Marsden y Weinstein 
[22]). Sin embargo, este camino plantea dificultades técnicas con las cuales no queremos lidiar.

La estrategia que seguimos en este trabajo es usar precisamente la reducción de Lie-Poisson. Así, después de plantear y discutir el problema de $N$-vórtices puntuales en el capítulo 4 nos concentramos en el problema de tres vórtices en el capítulo 5, donde planteamos las ecuaciones de movimiento en términos de los cuadrados de las distancias mutuas. Luego, siguiendo esta línea de ideas, en el capítulo 6 añadimos el área del triángulo formado por los vórtices como una variable independiente para definir un espacio vectorial de Poisson cuatro-dimensional. Identificando este espacio con el álgebra de Lie apropiada, implementamos una reducción que esencialmente consiste en la reducción de Lie-Poisson.

Elaboremos un poco más sobre las ideas contenidas en el párrafo anterior. Consideramos el espacio $\mathcal{V}$ definido por los cuadrados de la longitud de sus lados y su área. Como consecuencia de que éstas cantidades son invariantes de la acción de $G=S E(2)$, el paréntesis de Poisson en $\mathbb{C}^{3}$ asociado al problema de tres vórtices también es invariante cuando se evalúa en ellas. Esto nos permite inducir un paréntesis de Poisson en $\mathcal{V}$, que podemos pensar como un primer espacio reducido. La proyección $\pi: \mathbb{C}^{3} \rightarrow \mathcal{V}$ es una transformación de Poisson. Con ayuda de la matriz identidad de $2 \times 2$ y las matrices de Pauli, se observa que hay un isomorfismo entre $\mathcal{V}^{\star}$ y $\mathfrak{u}(2)$. Entonces la aplicación adjunta $\psi: \mathfrak{u}(2) \rightarrow \mathcal{V}^{\star}$ induce un isomorfismo entre álgebras de Lie. La dinámica en $\mathfrak{u}(2)^{*}$ se puede pensar como una dinámica de Lie-Poisson. Al igual que en el ejemplo del cuerpo rígido, las hojas simplécticas de $\mathfrak{u}(2)^{*}$ son invariantes de la dinámica. Un aspecto interesante es que, en contraste con lo que ocurre con en el ejemplo del cuerpo rígido, no todas las órbitas dinámicas en $\mathfrak{u}(2)^{*}$ tienen interpretación física. Las que sí lo tienen viven en ciertas hojas simplécticas determinadas por una condición íntimamente relacionada con la fórmula de Herón de la geometría clásica. En todo caso, al igual que en el ejemplo del cuerpo rígido, la dinámica se reduce a una órbita coadjunta de la acción de $U(2)$ sobre el dual de su álgebra de Lie. Las trayectorias dinámicas se obtienen finalmente intersecando esferas de radio constante con las superficies de nivel de la energía, en un hiperplano de un espacio de dimensión cuatro.

Al final del capítulo6 6interpretamos estas trayectorias dinámicas en términos de la forma simpléctica definida sobre la órbita coadjunta, dando cuatro soluciones particulares del sistema junto con el retrato fase correspondiente a un valor del momento angular. 
Es importante hacer notar que el concepto de reducción explorado en esta tesis está íntimamente relacionado con la noción integrabilidad del problema de tres vórtices. En la sección 3.6 discutimos este tema en su contexto histórico.

\section{Organización de la tesis}

La organización del presente trabajo es como sigue.

En el capítulo 2 comenzamos recordando algunas nociones de geometría diferencial. Se definen las variedades simplécticas y de Poisson. Lo anterior con la finalidad de entender las estructuras hamiltonianas definidas sobre este tipo de variedades. Se dan las definiciones de grupos y álgebras de Lie. Se describe como asociar un álgebra de Lie a un grupo de Lie, dando como ejemplos el grupo $U(2)$ y su álgebra $\mathbf{u}(2)$. Definimos el dual de un álgebra de Lie y las orbitas coadjuntas. Se describe la dinámica Hamiltoniana, tanto la formulación canónica y no canónica. Finalizamos el capítulo con la definición de Función Delta de Dirac.

En el capítulo 3 se dan las bases de la dinámica de vorticidad, pasando a la descripción de dicha dinámica en dos dimensiones. Tal dinámica bidimensional se ejemplifica con el flujo de punto fuente, flujo de vórtice puntual y flujo de punto esquina. A continuación se obtiene el campo de velocidad asociado a un vórtice puntual y damos las ecuaciones que describen la interacción de $N$ vórtices puntuales. El flujo asociado a un vórtice puntual se contrasta con otros dos ejemplos cuyos campos de velocidad hasta cierto punto se le asemejan: la rotación de cuerpo sólido y el vórtice de Rankine. Concluimos la sección discutiendo, en un contexto histórico, la integrabilidad del problema de tres vórtices.

En el capítulo 4 se describe la estructura hamiltoniana del problema de $N$ vórtices puntuales y se estudian dos casos especiales: el problema restringido de $N$ vórtices y las soluciones autosimilares.

En el capítulo 5 nos enfocamos en el problema de tres vórtices, dando las ecuaciones de movimiento en términos de las variables definidas por los cuadrados de las distancias entre los vórtices. Describimos el cono de las formas, que en estas variables es el espacio en el que, de acuerdo con la fórmula de Herón de geometría elemental, el problema está definido. Se dan las ecuaciones de movimiento definidas en este espacio y se observa que éstas no son hamiltonianas. 
En el capítulo 6 abordamos el álgebra y la reducción del problema de tres vórtices. Considerando el área como una variable independiente de la longitud de los lados del triángulo, lo cual nos permite definir una estructura de Poisson en $\mathbb{R}^{4}$, encajamos en este espacio el cono de las formas definido en el capítulo 5. Así, logramos obtener la dinámica reducida como una dinámica sobre órbitas coadjuntas (esferas). 


\section{Capítulo 2}

\section{Preliminares}

Este capítulo tiene por objeto repasar los conceptos básicos de geometía y dinámica hamiltoniana necesarios para estudiar la dinámica de vorticidad y el problema de $N$ vórtices.

\subsection{Variedades}

Esta sección tiene por objeto recordar algunas nociones de geometría diferencial, geometría simpléctica y estructuras de Poisson que se usarán más adelante. La referencia principal para esta sección es [9] y [24].

Comenzamos definiendo el concepto de variedad.

Definición 2.1.1. Una variedad diferenciable de dimensión n es un conjunto $M$ y una familia de aplicaciones inyectivas $f_{\alpha}: U_{\alpha} \longrightarrow M$, donde $U_{\alpha} \subset \mathbb{R}^{n}$ son abiertos de $R^{n}$ tales que:

1. $\bigcup_{\alpha} f_{\alpha}\left(U_{\alpha}\right)=M$

2. Para cualquier par $\alpha, \beta$ con $f_{\alpha}\left(U_{\alpha}\right) \cap f_{\beta}\left(U_{\beta}\right)=W \neq \emptyset$, los conjuntos $f_{\alpha}^{-1}(W)$ y $f_{\beta}^{-1}(W)$ son conjuntos abiertos en $\mathbb{R}^{n}$ y las aplicaciones $f_{\beta}^{-1} \circ f_{\alpha}$ son diferenciables.

3. La familia $\left\{\left(U_{\alpha}, f_{\alpha}\right)\right\}$ es maximal relativo a las condiciones 1 y 2 .

Así por ejemplo, si $U \subset \mathbb{R}^{n}$ es un abierto, entonces la aplicación $I d$ : $U \longrightarrow U$, es una variedad. 
Definición 2.1.2. Sea $F: U \longrightarrow \mathbb{R}^{m}$ una aplicación diferenciable de un conjunto abierto $U \subset \mathbb{R}^{n}$. Un punto $p \in U$ es un punto crítico de $F$ si la diferencial $d F_{p}: \mathbb{R}^{n} \longrightarrow \mathbb{R}^{m}$ no es suprayectiva. La imagen $F(p)$ de un punto crítico es llamado valor crítico de $F$. Un punto a $\in \mathbb{R}^{m}$ que no es crítico se dice que es valor regular de $F$.

Teorema 2.1.3. Sean $M^{m}, N^{n}$ variedades y $f \in C^{\infty}(M, N)$. Sea $q \in N$ tal que el rango de $f$ es una constante igual a $k$ en una vecindad de $f^{-1}(q) \neq \emptyset$. Entonces $f^{-1}(q)$ es una subvariedad de $M$, de dimensión $n-k$.

Cuando $k=m$, diremos que $q$ es un valor regular de $f$. El resultado anterior se suele enunciar diciendo que la imagen inversa de un valor regular es una subvariedad. En este caso, tenemos el siguiente hecho.

Teorema 2.1.4. Sea $f: N \longrightarrow M$, sea $q \in N$ un valor regular de $f \in$ $C^{\infty}\left(N^{n}, M^{m}\right)$. Para cada $p \in f^{-1}(q)$ se tiene que $T_{p} f^{-1}(q)=\operatorname{ker} d f_{p}$.

Notación pull-back para funciones. Si $\varphi: M \rightarrow M$ es diferenciable denotamos

$$
\varphi^{*} f:=f \circ \varphi
$$

para toda $f \in \mathcal{F}(M)$ (el conjunto de funciones suaves sobre $M$ ).

\subsubsection{Campos vectoriales.}

Un campo vectorial $X$ sobre una variedad $M$ es una función $X: M \rightarrow$ $T M$ que asigna un vector $X(m)$ al punto $m \in M$. El espacio vectorial real consistente de todos los campos vectoriales sobre $M$ se denota $\mathfrak{X}(M)$. Una curva integral de $X$ con condición inicial $m_{0}$ en $t=0$ es una curva diferenciable $c:[a, b] \rightarrow M$ tal que $(a, b)$ es un intervalo abierto conteniendo a $0, c(0)=m_{0}$, y tal que $c^{\prime}(t)=X(c(t))$ para todo $t \in(a, b)$.

El flujo de $X$ es la colección de funciones $\varphi_{t}: M \rightarrow M$ tales que $t \rightarrow$ $\varphi_{t}(m)$ es la única curva integral de $X$ con condición inicial $m$. (Los teoremas de existencia y unicidad para ecuaciones diferenciales ordinarias garantizan que si $X$ es suave entonces $\varphi$ es diferenciable en $m$ y $t$.) De la unicidad se tiene la propiedad de flujo

$$
\varphi_{t+s}=\varphi_{t} \circ \varphi_{s}
$$


junto con la condición inicial $\varphi_{0}=$ identidad.

Si $X$ es un campo vectorial sobre $M$ y $f \in \mathcal{F}(M)$ definimos la derivada direccional

$$
X[f](p)=\left.\frac{d}{d t}\right|_{t=0} f(\varphi(t))
$$

donde $\varphi(t)$ es la curva integral de $X$ con condición inicial $\varphi(0)=p$, con $\varphi^{\prime}(0)=X(p)$. (Una notación alternativa para $X[f]$ es $£_{X} f$, denotada la derivada de Lie de $f$ en la dirección de $X$. En este sentido decimos que un campo vectorial $X$ es una derivación que opera sobre $\mathcal{F}(M)$. Notemos que, en variedades de dimensión finita, el conjunto de derivaciones sobre $\mathcal{F}(M)$ coincide con $\mathfrak{X}(M)$.)

Notación pull-back para campos vectoriales. Si $\varphi: M \rightarrow M$ es un difeomorfismo y $Y \in \mathfrak{X}(M)$ entonces $\varphi^{*} Y$ denota el campo vectorial definido por

$$
\left(\varphi^{*} Y\right)(p)=\left(d \varphi^{-1} \circ Y \circ \varphi\right)(p) .
$$

Braquet de Lie. Dados dos campos vectoriales $X, Y \in \mathfrak{X}(M)$ definimos el braquet de Lie (o también llamado braquet de Jacobi-Lie) mediante

$$
[X, Y]=£_{X} Y:=\left.\frac{d}{d t}\right|_{t=0} \varphi^{*} Y
$$

donde $\varphi_{t}$ es el flujo de $X$. El símbolo $£_{X} Y$ se refiere como derivada de Lie de $Y$ con respecto a $X$.

Se tienen las siguientes propiedades (cf. [24, cap. 4]):

Proposición 2.1.5. Sean $X, Y, Z \in \mathfrak{X}(M), f \in \mathcal{F}(M)$ y $\varphi$ un difeomorfismo. Entonces

1. $[X, Y] \in \mathfrak{X}(M)$

2. $[X, Y][f]=X[Y[f]]-Y[X[f]]$. (En otras palabras, $[X, Y]$ es el conmutador de $X$ y $Y$.) En particular,

$$
[X, Y]=-[Y, X] .
$$

3. Se cumple la identidad de Jacobi:

$$
[[X, Y], Z]+[[Y, Z], X]+[[Z, X], Y]=0
$$

4. $\varphi^{*}[X, Y]=\left[\varphi^{*} X, \varphi^{*} Y\right]$. 


\subsection{Variedades simplécticas y de Poisson}

Una variedad de Poisson es un par $(M,\{\}$,$) que consiste de una variedad$ diferenciable con un corchete de Poisson $\{\}:, \mathbb{C}^{\infty}(M) \times \mathbb{C}^{\infty}(M) \longrightarrow \mathbb{C}^{\infty}(M)$, una operación $\mathbb{R}$-bilineal, antisimétrica que satisface la regla de Leibniz para el producto puntual de funciones suaves y para cualesquiera $f, g, h \in \mathbb{C}^{\infty}(M)$, se cumple la identidad de Jacobi:

$$
\{\{f, g\}, h\}+\{\{g, h\}, f\}+\{\{h, f\}, g\}=0 .
$$

Comenzaremos discutiendo las nociones de estructura de Poisson y forma simpléctica en el contexto de espacios vectoriales.

\subsubsection{Espacios vectoriales simplécticos y de Poisson}

Definición 2.2.1. Una forma simpléctica $\Omega$ sobre un espacio vectorial $V$ es una forma bilineal no-degenerada y antisimétrica sobre $V$. A la pareja $(V, \Omega)$ se le llama espacio vectorial simpléctico.

Dada $H \in \mathcal{F}(V)$, el campo vectorial Hamiltoniano $X_{H}$ queda definido por

$$
\Omega\left(X_{H}, \mathbf{v}\right)=d H \cdot \mathbf{v}
$$

A la ecuación (2.2) se le conoce como ecuación de Hamilton. Dado que $\Omega$ es no degenerada, no hay ambigüedad en la definición de $X_{H}$.

Definición 2.2.2. Dado un espacio vectorial simpléctico $(V, \Omega)$ y dos funciones $F, G: V \longrightarrow \mathbb{R}$, el braquet de Poisson $\{F, G\}: V \longrightarrow \mathbb{R}$ de $F$ y $G$ está definido por

$$
\{F, G\}(z)=\Omega\left(X_{F}(z), X_{G}(z)\right)
$$

Proposición 2.2.3. Sea $(V, \Omega)$ un espacio vectorial simpléctico. Entonces el braquet de Poisson $\{\}:, \mathcal{F}(V) \times \mathcal{F}(V) \longrightarrow \mathcal{F}(V)$ hace a $\mathcal{F}(V)$ un álgebra de Lie. Esto es, el braquet $\mathbb{R}$-bilineal, antisimétrica y satisface la identidad de Jacobi, esto es,

$$
\{\{F, G\} H\},\{\{G, H\} F\},\{\{H, F\} G\}=0
$$

En este contexto, otro resultado importante es el siguiente 
Proposición 2.2.4. Sea $\varphi: V \longrightarrow V$ un difeomorfismo. Entonces $\varphi$ es simpléctica si y sólo si se preserva el braquet de Poisson, es decir

$$
\left\{\varphi^{*} F, \varphi^{*} G\right\}=\varphi^{*}\{F, G\}
$$

para todo $F, G: V \longrightarrow \mathbb{R}$ diferenciable.

Definición 2.2.5. Una estructura de Poisson sobre una variedad $M$ es una operación bilineal $\{$,$\} sobre \mathcal{F}(M)=C^{\infty}(M)$ tal que:

1. $(\mathcal{F}(M),\{\}$,$) es una álgebra de Lie$

2. $\{$,$\} es una derivación en cada factor, es decir$

$$
\{F G, H\}=\{F, H\} G+F\{G, H\}
$$

para todo $F, G, H \in \mathcal{F}(M)$

Una variedad $M$ dotada con un braquet de Poisson sobre $\mathcal{F}(M)$ es llamada una variedad de Poisson. Si $M$ y $N$ son variedades de Poisson, decimos que $\varphi: M \longrightarrow N$ es una transformación de Poisson si se satisface (2.4).

Dada $\Omega$ una forma simpléctica, la matriz simpléctica $J$ determinada por $\Omega$ es la matriz definida por:

$$
\Omega(v, w)=v^{t} J w .
$$

En términos de la matriz simpléctica, el campo vectorial hamiltoniano $X_{f}$ asociado a un hamiltoniano $f$ viene dado por:

$$
\begin{aligned}
\Omega\left(X_{f}, w\right) & =d f \cdot w \\
J^{t} X_{f} & =\nabla f \\
X_{f} & =\left(J^{-1}\right)^{t} \cdot \nabla f
\end{aligned}
$$

por tanto tenemos que $X_{f}=\left(J^{-1}\right)^{t} \cdot \nabla f$.

Recordemos de la definición 2.2.2 que, dado un espacio vectorial simpléctico $(V, \Omega)$ y dos funciones $f, g: V \rightarrow \mathbb{R}$, el braquet de Poisson $\{f, g\}: V \rightarrow \mathbb{R}$ de $f$ y $g$ está definido por $\{f, g\}:=\Omega\left(X_{f}, X_{g}\right)$. Para la matriz simpléctica $J$, podemos calcular

$$
\begin{aligned}
\{f, g\} & =\Omega\left(X_{f}, X_{g}\right) \\
& =X_{f}^{t} J X_{g} \\
& =\left(\left(J^{-1}\right)^{t} \cdot \nabla f\right)^{t} J\left(\left(J^{-1}\right)^{t} \cdot \nabla g\right) \\
& =d f J^{-1} J\left(J^{-1}\right)^{t} \nabla g \\
& =d f\left(J^{-1}\right)^{t} \nabla g
\end{aligned}
$$


entonces para el braquet definido como $\{f, g\}=d f \cdot B \cdot \nabla g$, tenemos que nuestra matriz $B=\left(J^{-1}\right)^{t}$. En particular, si $J=\mathbb{J}$ (la matriz simpléctica usual) entonces $B$ coincide con $\mathbb{J}$.

Una variedad simpléctica es una variedad equipada con una dos forma $\Omega$ no degenerada y tal que $d \Omega=0$. El teorema de Darboux (ver [24, secc. 5.1]) garantiza que localmente siempre existe un sistema coordenado en donde la matriz simpléctica es constante y por lo tanto las expresiones para el campo vectorial hamiltoniano dadas arriba son válidas.

\subsubsection{Grupos y álgebras de Lie}

Definición 2.2.6. Un grupo de Lie es una superficie (de Banach) G tal que tiene estructura de grupo consistente con su estructura de superficie en el sentido de multiplicación de grupo

$$
\begin{aligned}
\mu: G \times G & \longrightarrow G \\
(g, h) & \mapsto g h
\end{aligned}
$$

es una aplicación $\mathbb{C}^{\infty}$.

Definición 2.2.7. Sea $X$ un espacio vectorial normado y sea d la métrica asociada. Si $X$ es un espacio métrico completo con respecto a d se dice que $X$ es un espacio de Banach.

Una superficie de Banach, es una variedad modelada sobre espacios de Banach, es decir, es un espacio topológico en el que cada punto tiene un entorno homeomorfo a un abierto de un espacio de Banach.

Las aplicaciones $L_{g}: G \longrightarrow G, h \mapsto g h$ y $R_{h}: G \longrightarrow G, g \mapsto g h$ son llamadas las aplicaciones izquierda y derecha. Notemos que

$$
\begin{aligned}
L_{g_{1}} \circ L_{g_{2}} & =L_{g_{1}}\left(L_{g_{2}}\right) \\
& =L_{g_{1}}\left(g_{2} h\right) \\
& =g_{1} g_{2} h \\
& =L_{g_{1} g_{2}}
\end{aligned}
$$

y $R_{h_{1}} \circ R_{h_{2}}=R_{h_{1} h_{2}}$

Si $e \in G$ denota al elemento identidad, entonces $L_{e}=I=R_{e}$ y también

$$
\begin{aligned}
\left(R_{h}\right)^{-1} & =(g h)^{-1} \\
& =h^{-1} g^{-1} \\
& =R_{h^{-1}}
\end{aligned}
$$


y $\left(L_{g}\right)^{-1}=L_{g^{-1}}$.

Así, $L_{g}$ y $R_{h}$ son difeomorfismos para cada $g$ y $h$. Notemos que

$$
\begin{aligned}
L_{g} \circ R_{h} & =L_{g}\left(R_{h}\right) \\
& =L_{g}(g h) \\
& =g g h \\
& =R_{h}(g h) \\
& =R_{h}\left(L_{g}\right) \\
& =R_{h} \circ L_{g}
\end{aligned}
$$

esto es, las aplicaciones izquierda y derecha conmutan. Por la regla de la cadena

$$
\begin{aligned}
T_{g h} L_{g^{-1}} \circ T_{h} L_{g} & =T_{h}\left(L_{g^{-1}} \circ L_{g}\right) \\
& =I d
\end{aligned}
$$

Así, $T_{h} L_{g}$ es invertible. Igualmente, $T_{g} R_{h}$ es un isomorfismo.

Consideremos la solución de

$$
\mu(g, h)=e
$$

para $h$ como función de $q$. La derivada parcial con respecto a $h$ es sólo $T_{h} L_{g}$ que es un isomorfismo. Así la solución $g^{-1}$ es una función suave de $g$ por el teorema de la función implícita, lo cuál nos dice que la aplicación $I: G \longrightarrow G$; $g \mapsto g^{-1}$ es $\mathbb{C}^{\infty}$.

En la subsección (2.2.4 hablaremos sobre el grupo de Lie $U(2)$ y su álgebra de Lie $\mathfrak{u}(2)$. Terminemos esta sección, entonces, definiendo lo que es un álgebra de Lie.

Definición 2.2.8. Un Álgebra de Lie sobre $\mathbb{R}$ es un espacio vectorial real $\mathfrak{g}$ con un operador bilineal $[]:, \mathfrak{g} \times \mathfrak{g} \longrightarrow \mathfrak{g}$ llamado braquet tal que para todo $x, y, z \in \mathfrak{g}$

$$
\begin{aligned}
& \text { 1. }[x, y]=-[y, x] \\
& \text { 2. }[[x, y], z]+[[y, z], x]+[[z, x], y]=0
\end{aligned}
$$

La propiedad (2) se la conoce como la identidad de Jacobi. 


\subsubsection{El álgebra de Lie asociada a un grupo de Lie}

En esta parte trataremos de explicar el como asociar un álgebra de Lie dado un grupo de Lie.

Un grupo de Lie es un grupo abstracto $G$ que tiene estructura de variedad diferenciable, respecto a la cual las aplicaciones $G \times G \rightarrow G ;(a, b) \mapsto a b$ y $G \rightarrow G ; a \mapsto a^{-1}$ son diferenciables.

Consideremos la traslación izquierda, es decir, si $G$ es un grupo de Lie y $a \in G$, la aplicación $L_{a}: G \rightarrow G, a \mapsto a g, g \in G$ es diferenciable; a $L_{a}$ se le denomina traslación izquierda.

Si $a, b \in G$ se verifica que $L_{a} \circ L_{b}=L_{a b}, L_{e}=i d, L_{a}^{-1}=L_{a^{-1}}$.

Si $G$ es un grupo de Lie, un campo $X \in \mathfrak{X}(G)$ se llama invariante por traslaciones izquierdas si $L_{a}^{*}(X)=X$ para todo $a \in G$, es decir $X(a g)=$ $d L_{a}(X(g))$ para todo $a, g \in G$. Denotaremos por $\mathcal{L}(G)$ al conjunto de dichos campos. Así, $X \in \mathcal{L}(G)$ precisamente cuando $L_{a}^{*} X=X$ para todo $a \in G$.

Es fácil ver que $\mathcal{L}(G)$ tiene estructura de álgebra de Lie, con braquet de Lie dado por el conmutador ó derivada de Lie heredado de $\mathfrak{X}(G)$ :

$$
[X, Y]=£_{X} Y, \quad X, Y \in \mathcal{L}(G) .
$$

(Recordemos que el conmutador ó derivada de Lie de un campo vectorial con respecto a otro se definió en (2.1).)

En efecto, de la proposición 2.1.5 se tiene que, si $X, Y \in \mathcal{L}(G)$,

$$
\begin{aligned}
L_{a}^{*}[X, Y] & =\left[L_{a}^{*} X, L_{a}^{*} Y\right] \\
& =[X, Y]
\end{aligned}
$$

para todo $a \in G$. Por lo tanto $[X, Y] \in \mathcal{L}(G)$. La identidad de Jacobi se cumple automáticamente (nuevamente por la proposición 2.1.5.)

El álgebra de Lie $\mathfrak{g}^{*}$ de un grupo $G$ se define como sigue. Como conjunto,

$$
\mathfrak{g}=T_{e} G,
$$

el espacio tangente a la identidad. Su braquet de Lie queda definido como sigue. Dados $\xi, \eta \in \mathfrak{g}^{*}$, sean $X_{\xi}, X_{\eta} \in \mathcal{L}(G)$ tales que

$$
X_{\xi}(e)=\xi, \quad X_{\eta}=\eta \text {. }
$$

Es decir, $X_{\xi}(a)=d L_{a} \cdot \xi$ para toda $a \in G$, y una expresión similar para $X_{\eta}$. Definimos el braquet de Lie en $\mathfrak{g}^{*}$ mediante

$$
[\xi, \eta]:=\left[X_{\xi}, X_{\eta}\right](e) .
$$


Es Claro que la identidad de Jacobi se satisface automáticamente. Notemos que $\mathfrak{g}^{*}$ y $\mathcal{L}(G)$ son isomorfos como espacios vectoriales y que, por construcción,

$$
\left[X_{\xi}, X_{\eta}\right]=X_{[\xi, \eta]} .
$$

Igual que se han definido las traslaciones izquierdas $L_{a}$, se pueden definir traslaciones derechas $R_{a}: G \rightarrow G, a \mapsto g a, g \in G$ y campos invariantes por traslaciones derechas, y los resultados son validos para $R_{a}$.

De esta manera podemos dotar a $\mathfrak{g}$ con dos braquets distintos: el braquet [, ] construido arriba y [, $]^{R}$, el braquet construido usando translaciones derechas. Se verifica que ambas están relacionadas por la ecuación

$$
[\xi, \eta]^{R}=-[\xi, \eta]
$$

Es claro que, con cualquiera de los dos braquets, $\mathfrak{g}$ satisface las propiedades de un álgebra de Lie dadas en la definición 2.2.8.

\subsection{4. $\quad$ El grupo $U(2)$ y su álgebra $\mathfrak{u}(2)$}

Recordemos que en $\mathbb{C}^{2}$ tenemos el producto Hermitiano, dado por

$$
\langle\mathbf{x}, \mathbf{y}\rangle=\sum_{i=0}^{2} x_{i} \bar{y}_{i}
$$

donde $\mathbf{x}=\left(x_{1}, x_{2}\right) \in \mathbb{C}^{2}, \mathbf{y}=\left(y_{1}, y_{2}\right) \in \mathbb{C}^{2}, \mathrm{y} \bar{y}_{i}$ denota el complejo conjugado. Sea

$$
U(2)=\{A \in G L(2, \mathbb{C}) \mid\langle A \mathbf{x}, A \mathbf{y}\rangle=\langle\mathbf{x}, \mathbf{y}\rangle\} .
$$

La condición de ortogonalidad $\langle A \mathbf{x}, A \mathbf{y}\rangle=\langle\mathbf{x}, \mathbf{y}\rangle$ es equivalente a $A A^{\dagger}=$ $A^{\dagger} A=I$, donde $A^{\dagger}=\bar{A}^{t}$, esto es $\langle A \mathbf{x}, \mathbf{y}\rangle=\left\langle\mathbf{x}, A^{\dagger} \mathbf{y}\right\rangle$. De $|\operatorname{det} A|=1$ podemos ver que el det aplica $U(n)$ en el circulo unitario $\mathbb{S}^{1}=\{z \in \mathbb{C}|| z \mid=1\}$.

Sean $A, B \in U(2)$, entonces

$$
\begin{aligned}
\langle A B \mathbf{x}, A B \mathbf{y}\rangle & =\langle B \mathbf{x}, B \mathbf{y}\rangle \\
& =\langle\mathbf{x}, \mathbf{y}\rangle
\end{aligned}
$$

entonces $A B \in U(2)$. Tomando $\mathbf{x}^{\prime}=A^{-1} \mathbf{x}$ y $\mathbf{y}^{\prime}=A^{-1} \mathbf{y}$, tenemos

$$
\begin{aligned}
\langle\mathbf{x}, \mathbf{y}\rangle & =\left\langle A \mathbf{x}^{\prime}, A \mathbf{y}^{\prime}\right\rangle \\
& =\left\langle\mathbf{x}^{\prime}, \mathbf{y}^{\prime}\right\rangle \\
& =\langle A \mathbf{x}, A \mathbf{y}\rangle
\end{aligned}
$$


entonces $A^{-1} \in U(2)$. De lo anterior tenemos que $U(2)$ es un subgrupo de Lie de $G L(2, \mathbb{C})$ con el álgebra de Lie dada por

$$
\begin{aligned}
\mathfrak{u}(2) & =\left\{A \in L\left(\mathbb{C}^{2}, \mathbb{C}^{2}\right) \mid\langle A \mathbf{x}, \mathbf{y}\rangle=-\langle\mathbf{x}, A \mathbf{y}\rangle\right\} \\
& =\left\{A \in \mathfrak{g l}(2, \mathbb{C}) \mid A^{\dagger}=-A\right\}
\end{aligned}
$$

Si $A=\left(\begin{array}{ll}x_{11}+i y_{11} & x_{12}+i y_{12} \\ x_{21}+i y_{21} & x_{22}+i y_{22}\end{array}\right) \in \mathfrak{u}(2)$ y por la condición $A=A^{\dagger}$, se tiene lo siguiente

$$
\left(\begin{array}{ll}
x_{11}-i y_{11} & x_{21}-i y_{21} \\
x_{12}-i y_{12} & x_{22}-i y_{22}
\end{array}\right)=\left(\begin{array}{ll}
-x_{11}-i y_{11} & -x_{12}-i y_{12} \\
-x_{21}-i y_{21} & -x_{22}-i y_{22}
\end{array}\right)
$$

de donde $x_{11}=x_{22}=0, x_{12}=-x_{21}$ y $y_{12}=y_{21}$, es decir, tenemos 4 parámetros libres, lo cual nos indica que la dimensión de $\mathfrak{u}(2)$ es igual a 4 , entonces la dimensión de $U(2)$ es 4 .

Sean $\tilde{\sigma}_{1}, \tilde{\sigma}_{2}, \tilde{\sigma}_{3}$ las matrices de Pauli, definidas por

$$
\tilde{\sigma}_{1}=-i\left(\begin{array}{cc}
0 & i \\
i & 0
\end{array}\right), \tilde{\sigma}_{2}=i\left(\begin{array}{cc}
0 & -1 \\
1 & 0
\end{array}\right) \text { y } \tilde{\sigma}_{3}=i\left(\begin{array}{cc}
-i & 0 \\
0 & i
\end{array}\right)
$$

junto con $\tilde{\sigma}_{0}=\left(\begin{array}{cc}i & 0 \\ 0 & i\end{array}\right)$ las cuales pertenecen a $\mathfrak{u}(2)$. Además cada una de las matrices satisface el que $\tilde{\sigma}_{i}^{\dagger}=-\tilde{\sigma}_{i}$, y son linealmente independientes. Por lo tanto son una base de $\mathfrak{u}(2)$. Para $i=1,2,3$, también satisfacen la propiedad de conmutación, es decir

$$
\left[\tilde{\sigma}_{i}, \tilde{\sigma}_{j}\right]=2 i \tilde{\sigma}_{k}(\text { siendo }(i, j, k) \text { permutación cíclica de }(1,2,3)) \text {. }
$$

Por la definición de centro de un álgebra de Lie, para $\mathfrak{u}(2)$ tenemos que

$$
\mathcal{Z}(\mathfrak{u}(2))=\{X \in \mathfrak{u}(2):[X, Y]=0 \forall Y \in \mathfrak{u}(2)\}
$$

entonces, como $\sigma_{0} \in \mathfrak{u}(2)$ consideramos

$$
\begin{aligned}
& {\left[\tilde{\sigma}_{0}, \tilde{\sigma}_{1}\right]=0} \\
& {\left[\tilde{\sigma}_{0}, \tilde{\sigma}_{2}\right]=0} \\
& {\left[\tilde{\sigma}_{0}, \tilde{\sigma}_{3}\right]=0}
\end{aligned}
$$

de donde observamos que el centro está generado por $\tilde{\sigma}_{0}$. 


\subsubsection{El dual de una álgebra de Lie}

Dado un espacio vectorial $V$, su dual $V^{*}$ es el conjunto de funcionales lineales $\mu: V \longrightarrow \mathbb{R}$. El dual de un álgebra de Lie $\mathfrak{g}$ se denota, desde luego, mediante $\mathfrak{g}^{*}$.

Dado un producto interno en $\mathfrak{g}$, que denotaremos mediante $\langle$,$\rangle , sea$

$$
T: \mathfrak{g} \longrightarrow \mathfrak{g}^{*}
$$

el isomorfismo inducido dado por $\langle T(\xi), \eta\rangle=\langle\xi, \eta\rangle$.

En lo que sigue fijaremos un producto interno en $\mathfrak{g}$, el álgebra de Lie de un grupo matricial, mediante

$$
\langle\xi, \eta\rangle=\operatorname{tr}(\xi \cdot \eta) .
$$

\subsection{6. Órbitas coadjuntas y foliación simpléctica}

La representación adjunta de un grupo de Lie $G$ está definida por

$$
A d_{g}=d\left(I_{g}\right)_{e}: \mathfrak{g} \longrightarrow \mathfrak{g}
$$

donde $I_{g}: G \longrightarrow G$ es el automorfismo interno $I_{g}(h)=g h g^{-1}$. Se define (ver [24, Capítulo 14]) la acción coadjunta de $G$ sobre $\mathfrak{g}^{*}$ mediante

$$
A d_{g^{-1}}^{*}: \mathfrak{g}^{*} \longrightarrow \mathfrak{g}^{*}
$$

donde $A d_{g^{-1}}^{*}$ es el dual de la aplicación $A d_{g^{-1}}$, es decir, está definida mediante

$$
\left\langle A d_{g^{-1}}^{*}(\mu), \xi\right\rangle=\left\langle\mu, A d_{g^{-1}}(\xi)\right\rangle
$$

donde $\mu \in \mathfrak{g}^{*}, \xi \in \mathfrak{g} \mathrm{y}\langle$,$\rangle denota el pareamientd 1$ entre $\mathfrak{g}^{*}$ y $\mathfrak{g}$.

Para un grupo de Lie matricial

$$
\begin{aligned}
\left(d I_{g}\right)_{e} \cdot \xi & =\left.\frac{d}{d t}\right|_{t=0} I_{g}(e+t \xi) \\
& =\left.\frac{d}{d t}\right|_{t=o}\left[g(e+t \xi) g^{-1}\right] \\
& =\left.\frac{d}{d t}\right|_{t=0}\left[g g^{-1}+t g \xi g^{-1}\right] \\
& =g \xi g^{-1}
\end{aligned}
$$

\footnotetext{
${ }^{1}$ Notar que usamos paréntesis angulares para denotar tanto el pareamiento entre el álgebra y su dual como el producto interno en el álgebra.
} 
Sea $T$ el isomorfismo entre $\mathfrak{g}$ y $\mathfrak{g}^{*}$ definido por un producto interno en $\mathfrak{g}$. Dado $\mu=T(\xi) \in \mathfrak{g}^{*}$, observamos que

$$
\begin{aligned}
\left\langle\operatorname{Ad}_{g^{-1}}^{*}(\mu), \eta\right\rangle & =\left\langle T(\xi), \operatorname{Ad}_{g^{-1}}(\eta)\right\rangle=\left\langle\xi, g^{-1} \eta g\right\rangle \\
& =\operatorname{tr}\left(\xi \cdot g^{-1} \eta g\right)=\operatorname{tr}\left(g \xi g^{-1} \cdot \eta\right) \\
& =\left\langle g \xi g^{-1}, \eta\right\rangle=\left\langle T\left(\operatorname{Ad}_{g}(\xi)\right), \eta\right\rangle
\end{aligned}
$$

por lo que el siguiente diagrama es conmutativo:

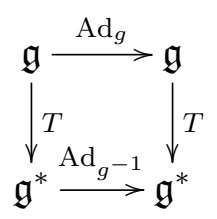

En otras palabras, el isomorfismo $T$ identifica la acción coadjunta en $\mathfrak{g}^{*}$ con la acción adjunta en $\mathfrak{g}$.

La órbita coadjunta $\operatorname{Orb}(\mu)$, a lo largo de $\mu \in \mathfrak{g}^{*}$ es el subconjunto de $\mathfrak{g}^{*}$ definido por

$$
\operatorname{Orb}(\mu):=\left\{A d_{g^{-1}}^{*}(\mu) \mid g \in G\right\}:=G \cdot \mu
$$

Parecido a la orbita de cualquier acción de grupo, $\operatorname{Orb}(\mu)$ es una subvariedad inmersa de $\mathfrak{g}^{*}$, y si $G$ es compacto, $\operatorname{Orb}(\mu)$ es una subvariedad.

Dado que las orbitas coadjuntas son simplécticas, tenemos de [24, Cap. 14] lo siguiente:

Teorema 2.2.9 (Orbita Coadjunta). Sea $G$ un grupo de Lie y sea $\mathcal{O} \subset \mathfrak{g}^{*}$ una orbita coadjunta. Entonces

$$
\omega^{ \pm}(\mu)\left(\xi_{\mathfrak{g}^{*}}(\mu), \eta_{\mathfrak{g}^{*}}(\mu)\right)= \pm\langle\mu,[\xi, \eta]\rangle
$$

para todo $\mu \in \mathcal{O}$ y $\xi, \eta \in \mathfrak{g}$ define formas simplécticas sobre $\mathcal{O}$. Referimos a $\omega^{ \pm}$como la estructura de órbita coadjunta simpléctica, denotada por $\omega_{\mathcal{O}}^{ \pm}$.

Proposición 2.2.10. Las órbitas coadjuntas de grupos de Lie finito-dimensional son de dimensión par.

Proposición 2.2.11. Sea $G$ un grupo de Lie matricial. Entonces el determinante y la traza, como funciones en $\mathfrak{g}^{*}$, son invariantes de la acción coadjunta. 
Demostración. Dado $\mu \in \mathfrak{g}^{*}$, sea $\mathcal{O}_{\mu}=\left\{g^{-1} \mu g \mid g \in G\right\}$ orbita coadjunta. Calculamos

$$
\begin{aligned}
\operatorname{det}\left(g^{-1} \mu g\right) & =\operatorname{det}\left(g^{-1}\right) \operatorname{det}(\mu) \operatorname{det}(g) \\
& =\operatorname{det}(\mu) \quad \forall g \in G
\end{aligned}
$$

y

$$
\begin{aligned}
\operatorname{tr}\left(g^{-1} \mu g\right) & =\operatorname{tr}\left(\mu g g^{-1}\right) \\
& =\operatorname{tr}(\mu) \forall g \in G .
\end{aligned}
$$

Por lo tanto tenemos que el determinante y la traza son invariantes en la órbita coadjunta.

\subsection{7. Órbitas coadjuntas en $\mathfrak{u}(2)^{*}$}

Definamos la función $\tilde{F}: \mathbb{R}^{4} \longrightarrow \mathbb{R}^{2}$ mediante

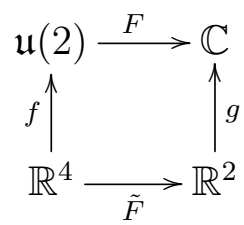

donde $F(\mu)=(\operatorname{det}(\mu), \operatorname{tr}(\mu)), g(x, y)=x+i y, \mathrm{y} f$ es la parametrización de $\mathfrak{u}(2)^{*}$ dada por

$$
f\left(x_{1}, x_{2}, x_{3}, x_{4}\right)=\left(\begin{array}{cc}
i x_{1} & \frac{x_{2}+i x_{3}}{\sqrt{2}} \\
\frac{-x_{2}+i x_{3}}{\sqrt{2}} & i x_{4}
\end{array}\right) .
$$

Calculando que $\operatorname{det}\left(f\left(x_{1}, x_{2}, x_{3}, x_{4}\right)\right)=\frac{1}{2}\left(x_{2}^{2}+x_{3}^{2}-2 x_{1} x_{4}\right)$ y la traza $\operatorname{tr}\left(f\left(x_{1}, x_{2}, x_{3}, x_{4}\right)\right)=i\left(x_{1}+x_{4}\right)$, obtenemos

$$
D \tilde{F}=\left(\begin{array}{cccc}
-x_{4} & x_{2} & x_{3} & -x_{1} \\
1 & 0 & 0 & 1
\end{array}\right) .
$$

Ahora, para poder determinar la dimensión del rango de $D \tilde{F}$, tomemos la siguiente combinación lineal

$$
\begin{array}{r}
-\alpha_{1} x_{4}+\alpha_{2} x_{2}+\alpha_{3} x_{3}-\alpha_{4} x_{1}=0 \\
\alpha_{1}+\alpha_{4}=0
\end{array}
$$


y observamos que las condiciones para que $D \tilde{F}$ no tenga rango máximo están dadas por el conjunto

$$
\mathrm{PC}=\left\{\left(x_{1}, x_{2}, x_{3}, x_{4}\right) \in \mathbb{R}^{4} \mid x_{2}=0, x_{3}=0, x_{1}-x_{4}=0\right\} .
$$

El conjunto anterior es el conjunto de valores críticos de $\tilde{F}$, considerando $\tilde{F}(t, 0,0, t)=\left(-t^{2}, 2 t\right)$, lo que nos indica que el conjunto de valores críticos de $\tilde{F}$ es una parábola.

Por tanto la órbita coadjunta que pasa por cualquier punto regular tiene $\operatorname{dim}>0$. En efecto, la dimensión de la órbita coadjunta no es cero, ya que la matriz Jacobiana de la aplicación $\tilde{F}$ es distinta de la matriz cero. Entonces tenemos que $\operatorname{dim} \supsetneqq 0$.

Sea $\left(d_{0}, t_{0}\right)$ un valor regular de $\tilde{F}$. Si $\mathbf{x}=\left(x_{1}, x_{2}, x_{3}, x_{4}\right) \in \tilde{F}^{-1}\left(d_{0}, t_{0}\right)$ entonces, por la proposición 2.2.11, la órbita coadjunta que pasa por $\mathbf{x}$ está contenida en $\tilde{F}^{-1}\left(d_{0}, t_{0}\right)$. Por el teorema 2.1.4 tenemos que la $\operatorname{dim} \tilde{F}^{-1}\left(d_{0}, t_{0}\right)=$ 2. Por tanto:

Proposición 2.2.12. Sea $\mu \in \mathfrak{u}(2)^{*}$ un punto regular de $\tilde{F}$. Sea $\mathcal{O}_{\mu}$ la órbita coadjunta que contiene a $\mu$. Entonces $\operatorname{dim} \mathcal{O}_{\mu}=2$.

Demostración. La dimensión de la órbita coadjunta, que es par, no es cero y, por estar contenida en $\tilde{F}^{-1}\left(d_{0}, t_{0}\right)$, es menor ó igual a dos. Hemos visto que $\mathcal{O}_{\mu} \subset \tilde{F}^{-1}\left(d_{0}, t_{0}\right)$. Se verifica que, de hecho, se da la igualdad. Para ello nos apoyamos en el siguiente hecho: si $f: M \longrightarrow N$ es una inmersión, $M$ es compacta y $N$ es convexa, entonces $f$ es suprayectiva (cf. el ejercicio 13 del capítulo 1 de [37]). Como $i: \mathcal{O}_{\mu} \longrightarrow \tilde{F}^{-1}\left(d_{0}, t_{0}\right)$ es una inmersión, entonces $i$ es suprayectiva. Por lo tanto $\mathcal{O}_{\mu}=\tilde{F}^{-1}\left(d_{0}, t_{0}\right)$, es decir tenemos la igualdad deseada.

\subsection{Dinámica hamiltoniana}

En esta sección revisaremos las formulaciones canónica y no-canónica de la dinámica hamiltoniana. En la primera, típicamente el hamiltoniano se define como la suma de las energías cinética y potencial. Las ecuaciones de Hamilton quedan en ese caso determinadas por el braquet canónico de Poisson en una variedad de dimensión par. Veremos también cómo la dinámica Hamiltoniana se puede definir usando estructuras de Poisson más generales donde ya no se requiere que la dimensión de la variedad sea par. 


\subsubsection{Formulación Canónica}

La ecuación canónica Hamiltoniana, dada la función

$$
\mathcal{H}(\mathbf{q}, \mathbf{p}) \equiv \mathcal{H}(\mathbf{z})
$$

donde $\mathbf{z} \equiv\left(q_{1}, \ldots, q_{n}, p_{1}, \ldots, p_{n}\right) \in \mathbb{R}^{2 n}$, toma la forma

$$
\dot{\mathbf{z}}=\mathbb{J} \cdot \nabla \mathcal{H}(\mathbf{z})
$$

donde $\nabla \equiv\left(\partial z_{1}, \ldots, \partial z_{n}\right)$ es el operador gradiente en $\mathbb{R}^{2}$ y $\mathbb{J}$ es la matriz simpléctica de tamaño $2 n \times 2 n$

$$
\mathbb{J}=\left(\begin{array}{cc}
0 & \overline{1} \\
\overline{1} & 0
\end{array}\right) \text { donde } \overline{1} \text { es la matriz identidad de } 2 n \times 2 n .
$$

Definición 2.3.1. Una matriz $\mathbb{T}$ es simpléctica si $\mathbb{T}^{t} \mathbb{J}=\mathbb{J}$.

Notemos que $\mathbb{J}$ es simpléctica, dada $\mathbb{J}^{-1}=\mathbb{J}^{t}\left(\mathbb{J}=\left(\mathbb{J}^{t}\right)^{-1}\right)$ tenemos $\mathrm{Id}=\mathbb{J}^{t} \mathbb{J}$.

Al lado derecho de (2.14) se le llama el gradiente simpléctico de $\mathcal{H}$.

Veamos como se define el Hamiltoniano mediante la suma de la Energía Potencial más Cinética.

1. Comenzamos por escribir adecuadamente el lagrangiano $L(\mathbf{q}, \dot{\mathbf{q}} ; t)=$ $T-V$ en términos de la energía cinética $T$ y la energía potencial $V$. De esto podemos definir la integral

$$
W=\int_{t_{1}}^{t_{2}} L(\mathbf{q}, \dot{\mathbf{q}} ; t)
$$

de donde obtenemos a partir del principio variacional $\delta W=0$ las ecuaciones de movimiento

$$
\frac{d}{d t} \frac{\partial L}{\partial \dot{q}_{i}}-\frac{\partial L}{\partial q_{i}}=0
$$

Es fácil mostrar que si $L$ no depende explícitamente del tiempo, la energía de el sistema

$$
\begin{aligned}
E & =T+V \\
& =\sum_{i} \dot{q}_{i} \frac{\partial L}{\partial \dot{q}_{i}}-L
\end{aligned}
$$

es constante. 
2. Tomando un cambio de variables (llamada transformación de Legendre), introducimos $p_{i}=\frac{\partial L}{\partial \dot{q}_{i}}$, entonces la energía del sistema puede ser escrita como

$$
E=\sum_{i=1}^{n} p_{i} \dot{q}_{i}-L
$$

Para escribir explícitamente los $\dot{q}_{i}$ en términos de los $p_{i}$ requerimos que $\left|\frac{\partial^{2} L}{\partial \dot{q}_{i} \partial \dot{q}_{j}}\right| \neq 0$.

El espacio definido por las variables $(\mathbf{q}, \mathbf{p})$ es llamado el espacio fase 2 dimensional del sistema. El Hamiltoniano es definido como la energía

$$
\mathcal{H}(\mathbf{q}, \mathbf{p}, t)=\sum_{i=1}^{n} p_{i} \dot{q}_{i}-L(\mathbf{q}, \dot{\mathbf{q}} ; t), \text { donde } p_{i}=\frac{\partial L}{\partial \dot{q}_{i}},
$$

que es la suma de la energía potencial más la cinética.

3. De la definición de $\mathcal{H}$, obtenemos

$$
d \mathcal{H}=\sum\left(\dot{q}_{i} d p_{i}-\dot{p}_{i} d q_{i}\right)-\frac{\partial L}{\partial t}
$$

para obtener las ecuaciones de Hamilton en forma canónica

$$
\dot{q}_{i}=\frac{\partial \mathcal{H}}{\partial p_{i}}, \quad \dot{p}_{i}=-\frac{\partial \mathcal{H}}{\partial \dot{q}_{i}}
$$

4. El teorema de Liouville establece que el volumen en el espacio fase se conserva

$$
\begin{aligned}
\nabla \cdot(\dot{z}) & =\nabla(\mathbb{J} \cdot \nabla \mathcal{H}) \\
& =\sum_{i=1}^{n} \frac{\partial}{\partial q_{i}}\left(\frac{\partial \mathcal{H}}{\partial p_{i}}\right)+\frac{\partial}{\partial p_{i}}\left(-\frac{\partial \mathcal{H}}{\partial q_{i}}\right) \\
& =0
\end{aligned}
$$

\subsubsection{Braquet de Poisson}

Consideremos la derivada con respecto al tiempo de una función de clase $C^{1}, f(\mathbf{q}, \mathbf{p} ; \mathbf{t})$ definida sobre el espacio fase 2 n-dimensional. Por la regla de la cadena tenemos que

$$
\frac{d f}{d t}=\sum_{i}\left(\frac{\partial f}{\partial q_{i}} \frac{\partial q_{i}}{\partial t}+\frac{\partial f}{\partial p_{i}} \frac{\partial p_{i}}{\partial t}\right)+\frac{\partial f}{\partial t} d t
$$


y sabemos que

$$
\begin{aligned}
\frac{\partial q_{i}}{\partial t} & =\dot{q}_{i} \\
& =\frac{\partial \mathcal{H}}{\partial p_{i}}
\end{aligned}
$$

y también

$$
\begin{aligned}
\frac{\partial p_{i}}{\partial t} & =\dot{p}_{i} \\
& =-\frac{\partial \mathcal{H}}{\partial q_{i}}
\end{aligned}
$$

entonces tenemos

$$
\begin{aligned}
\frac{\partial f}{\partial t} & =\sum_{i}\left(\frac{\partial f}{\partial q_{i}} \frac{\partial \mathcal{H}}{\partial p_{i}}-\frac{\partial f}{\partial p_{i}} \frac{\partial \mathcal{H}}{\partial q_{i}}\right)+\frac{\partial f}{\partial t} d t \\
& \equiv\{f, \mathcal{H}\}+\frac{\partial f}{\partial t} d t .
\end{aligned}
$$

De donde el último paso define el braquet canónico de Poisson de 2 funciones, es decir

$$
\{f, g\} \equiv \sum\left(\frac{\partial f}{\partial q_{i}} \frac{\partial g}{\partial p_{i}}-\frac{\partial f}{\partial p_{i}} \frac{\partial g}{\partial q_{i}}\right)
$$

Con este braquet, $\mathbb{R}^{2 N}$ es una variedad de Poisson en el sentido de la sección 2.2. La fórmula (2.16) muestra que si la función $f$ es independiente del tiempo y si el braquet de Poisson con el Hamiltoniano se anula, entonces es una constante de la integral de movimiento.

Usando esta definición, se tienen las siguientes identidades del braquet de Poisson:

1. $\{f, g\}=-\{g, f\}$

2. $\{f+g, h\}=\{f, h\}+\{g, h\}$

3. Identidad de Jacobi

$$
\{f,\{g, h\}\}+\{g,\{h, f\}\}+\{h,\{f, g\}\}=0
$$


4. Identidad de Leibniz

$$
\{f g, h\}=f\{g, h\}+g\{f, h\}
$$

Las identidades (1), (2) y (3) definen una Álgebra de Lie

Ejemplo 2.3.2. $\{\mathcal{H}, \mathcal{H}\}=0$, de aquí el Hamiltoniano es una cantidad conservada, $\mathcal{H}=E$ para sistemas independientes del tiempo.

Teorema 2.3.3. Si dos funciones $f$ y g son cantidades conservadas, entonces $\{f, g\}$ es también una cantidad conservada.

Demostración. Sólo basta usar la identidad de Jacobi con $f, g$ y $\mathcal{H}$ para mostrar que $\{\mathcal{H},\{f, g\}\}=0$.

Definición 2.3.4. Dos funciones que se cancelan en el braquet de Poisson, se dice que están en involución (ó que son involutivas). Equivalentemente se dice que Poisson-conmutan.

Dada una función $F$ de clase $C^{\infty}$ definida sobre el espacio fase $(\mathbf{q}, \mathbf{p})$ de un sistema Hamiltoniano, las ecuaciones de Hamilton son

$$
\begin{aligned}
\dot{F} & =\sum_{i}\left[\frac{\partial F}{\partial q_{i}} \frac{\partial q_{i}}{\partial t}+\frac{\partial F}{\partial p_{i}} \frac{\partial p_{i}}{\partial t}\right] \\
& =\sum_{i}\left[\frac{\partial F}{\partial q_{i}} \frac{\partial \mathcal{H}}{\partial p_{i}}-\frac{\partial F}{\partial p_{i}} \frac{\partial \mathcal{H}}{\partial q_{i}}\right] \\
& =\{F, \mathcal{H}\} .
\end{aligned}
$$

Tomando a $F=q_{i}$ y $F=p_{i}$ obtenemos las ecuaciones Hamiltonianas clásicas 2.15).

Las propiedades 1., 2., 3., 4. mencionadas arriba para el braquet de Poisson canónico definen de hecho el concepto de braquet de Poisson en un espacio vectorial. En general, dado un braquet de Poisson y un hamiltoniano (que no dependa explícitamente del tiempo), las ecuaciones de Hamilton que definen la dinámica son, por definición

$$
\dot{F}=\{F, \mathcal{H}\}
$$




\subsubsection{Formulación no canónica}

El significado de la idea del desarrollo de la mecánica no canónica comienza con la generalización de la definición de braquet de Poisson. Consideremos el ejemplo de la dinámica del cuerpo rígido.

Ejemplo 2.3.5. Las ecuaciones de movimiento del cuerpo rígido dinámico son

$$
\begin{aligned}
& I_{1} \dot{\Omega}_{1}=\left(I_{2}-I_{3}\right) \Omega_{2} \Omega_{3} \\
& I_{2} \dot{\Omega}_{2}=\left(I_{3}-I_{1}\right) \Omega_{3} \Omega_{1} \\
& I_{3} \dot{\Omega}_{3}=\left(I_{1}-I_{2}\right) \Omega_{1} \Omega_{2}
\end{aligned}
$$

donde $\Omega \equiv\left(\Omega_{1}, \Omega_{2}, \Omega_{3}\right)$ es la velocidad angular de el cuerpo rígido de un cuerpo fijo de marco de referencia, e $I \equiv\left(I_{1}, I_{2}, I_{3}\right)$ son los momentos principales de inercia del cuerpo. Dado que el sistema está compuesto por 3 ecuaciones, es claro que no es una forma canónica.

Definimos el momento angular

$$
\Pi \equiv\left(\Pi_{1}, \Pi_{2}, \Pi_{3}\right)=\left(I_{1} \Omega_{1}, I_{2} \Omega_{2}, I_{3} \Omega_{3}\right)
$$

Entonces las ecuaciones del cuerpo rígido se pueden poner en forma compacta como

$$
\dot{\Pi}=\Pi \times \Omega .
$$

Podemos definir el braquet de Poisson del cuerpo rígido, que actúa sobre las funciones del momento angular como

$$
\{F, G\}=-\Pi \cdot(\nabla F \times \nabla G) .
$$

Se puede verificar que el sistema 2.21 es equivalente a

$$
\dot{F}=\{F, \mathcal{H}\}
$$

con el Hamiltoniano $\mathcal{H}=\frac{1}{2}\left(\frac{\Pi_{1}^{2}}{I_{1}}+\frac{\Pi_{2}^{2}}{I_{2}}+\frac{\Pi_{3}^{2}}{I_{3}}\right)$. Tomando $F$ como $\Pi_{1}, \Pi_{2}$ y $\Pi_{3}$ en turno, tenemos que

$$
\left\{\Pi_{i}, \mathcal{H}\right\}=\dot{\Pi}_{i} \quad i=1,2,3 .
$$

Notemos que si tomamos el momento angular total

$$
\mathcal{C}(\Pi) \equiv \frac{1}{2}\left(\Pi_{1}^{2}+\Pi_{2}^{2}+\Pi_{3}^{2}\right)
$$


y lo ocupamos en

$$
\begin{aligned}
\dot{\mathcal{C}} & =\{\mathcal{C}, \mathcal{H}\} \\
& =-\Pi \cdot(\nabla \mathcal{C} \times \nabla \mathcal{H}) \\
& =-\Pi \cdot(\Pi \times \nabla \mathcal{H}) \\
& =0
\end{aligned}
$$

obtenemos una aseveración de conservación de momento angular. De hecho para cualquier función $G$ se puede probar

$$
\{\mathcal{C}, G\}=0
$$

es decir $\mathcal{C}$ Poisson-conmuta con todas las funciones.

Esto motiva la definición que se llama funciones Casimir:

Definición 2.3.6. Una función Casimir $\mathcal{C}$ es una función diferenciable tal que Poisson-conmuta con cualquier función $G$, es decir

$$
\{\mathcal{C}, G\}=0
$$

El ejemplo del cuerpo rígido es un caso particular de una estructura de Poisson definida sobre el dual de un álgebra de Lie $\mathfrak{g}^{*}$. De acuerdo con [24, Capítulo 10] ésta se define como:

$$
\{F, G\}(\mu)=\left\langle\mu,\left[\frac{\delta F}{\delta \mu}, \frac{\delta G}{\delta \mu}\right]\right\rangle
$$

para $\mu \in \mathfrak{g}^{*}$.

\subsection{Función $\delta$ de Dirac}

En esta sección discutiremos la función $\delta$ de Dirac, la cual definiremos mediante el límite de una sucesión de funciones. La función $\delta$ de Dirac no es una función en el sentido usual del Cálculo, pues se sabe que una función idénticamente nula excepto un punto tiene siempre integral cero, en lugar de uno, es decir

$f(x)=0, \forall x \neq a \Rightarrow \int_{-\infty}^{\infty} f(x) d x=\int_{-\infty}^{a} f(x) d x+\int_{a}^{\infty} f(x) d x=0, a \in \mathbb{R}$ 
Lo anterior significa que no es posible aplicar los resultados del Cálculo a la $\delta$ de Dirac.

Comencemos considerando las sucesiones siguientes:

$$
\begin{gathered}
s_{n}(t)=\left\{\begin{array}{lc}
1 & t>1 / 2 n \\
n t+1 / 2 & -1 / 2 n \leq t \leq 1 / 2 n \\
0 & t<-1 / 2 n
\end{array}\right. \\
d_{n}(t)=\left\{\begin{array}{lc}
0 & t>1 / 2 n \\
n & -1 / 2 n<t<1 / 2 n \\
0 & t<-1 / 2 n
\end{array}\right.
\end{gathered}
$$

observamos que

1. $\left.\frac{d}{d t} s_{n}(t)\right|_{t_{0}}=d_{n}\left(t_{0}\right), t_{0} \neq \pm 1 / 2 n$

2. Por como se definió $s_{n}, d_{n}$ y por el Teorema Fundamental del Cálculo

$$
\int_{-\infty}^{t_{0}} d_{n}(t) d t=s_{n}\left(t_{0}\right) \text { para todo } t_{0}
$$

3. $\int_{-\infty}^{\infty} f(t) d_{n}(t) d t=f(\xi),-1 / 2 n<\xi<1 / 2 n$

Si hacemos tender $n$ a infinito para las sucesiones $s_{n}$ y $d_{n}$, tenemos

$$
\lim _{n \rightarrow \infty} s_{n}(t)=u(t)= \begin{cases}0 & t<0 \\ 1 & t>0\end{cases}
$$

y

$$
\lim _{n \rightarrow \infty} d_{n}(t)=d_{\infty}(t)= \begin{cases}\infty & t=0 \\ 0 & t \neq 0\end{cases}
$$

entonces, tenemos

$$
\int_{-\infty}^{\infty} f(t) d_{\infty}(t) d t=0
$$

ahora, si consideramos

$$
\begin{aligned}
\lim _{n \rightarrow \infty} \int_{-\infty}^{\infty} f(t) d_{n}(t) d t & =\lim _{n \rightarrow \infty} f(\xi) \\
& =f(0)
\end{aligned}
$$

esto nos dice cómo debemos definir la $\delta$ de Dirac: 
Definición 2.4.1. La función Delta de Dirac $\delta\left(x, x_{0}\right)$ se define como

$$
\delta\left(x, x_{0}\right) \equiv \lim _{n \rightarrow \infty} d_{n}\left(x-x_{0}\right)
$$

y tiene las siguientes propiedades:

$$
\begin{aligned}
\int_{-\infty}^{\infty} \delta\left(x, x_{0}\right) d x & =1, \\
\int_{-\infty}^{\infty} f(x) \delta\left(x, x_{0}\right) d x & =f\left(x_{0}\right) .
\end{aligned}
$$

Es fácil generalizar la definición anterior a dos (ó más) dimensiones. Para definir la Delta de Dirac en dos dimensiones, substituimos las propiedades (2.23) y (2.24), respectivamente, por:

$$
\iint_{\mathbb{R}^{2}} \delta\left(\mathbf{x}, \mathbf{x}_{0}\right) d x d y=1 \quad \text { y } \iint_{\mathbb{R}^{2}} f(\mathbf{x}) \delta\left(\mathbf{x}, \mathbf{x}_{0}\right) d x d y=f\left(\mathbf{x}_{0}\right) .
$$




\section{Capítulo 3}

\section{Dinámica de vorticidad}

En este capítulo estableceremos las bases de la dinámica de un fluido ideal en términos del campo de vorticidad y las ecuaciones de movimiento que gobiernan la dinámica de $N$ vórtices puntuales.

\subsection{Nociones básicas}

Dado un campo de flujo con velocidad de distribución $\mathbf{u}=(u, v, w) \in \mathbb{R}^{3}$, el campo de vorticidad asociado es

$$
\omega=\nabla \times \mathbf{u} .
$$

La ecuación (3.1) es conocida como el rotacional en $\mathbb{R}^{3}$. Nos ocuparemos exclusivamente de fluidos no viscosos, de flujos incompresibles con constante (normalizada) de densidad $\rho=1$. La condición de incompresibilidad esta dada por

$$
\nabla \cdot \mathbf{u}=0
$$

Tomando la divergencia y el rotacional de (3.1) tenemos las relaciones

$$
\begin{aligned}
\nabla \cdot \omega & =0 \\
\nabla^{2} \mathbf{u} & =-\nabla \times \omega .
\end{aligned}
$$

La primera relación es clara. Para la segunda nos basta recordar que

$$
\nabla \times(\nabla \times \mathbf{u})=\nabla(\nabla \cdot \mathbf{u})-\nabla^{2} \mathbf{u}
$$


y bajo la condición de incompresibilidad $\nabla \cdot \mathbf{u}=0$ de donde obtenemos lo deseado, es decir

$$
\nabla \times(\nabla \times \mathbf{u})=-\nabla^{2} \mathbf{u}
$$

La ecuación (3.3) muestra que las componentes de vorticidad están relacionadas unas con otras de la misma forma que las componentes de velocidad, mientras que (3.4) muestra que cada componente de velocidad satisface una ecuación de Poisson. Si la divergencia de la vorticidad es integrada sobre un volumen finito $V \in \mathbb{R}^{3}$ el teorema de la divergencia nos dice

$$
\int_{V} \nabla \cdot \omega d V=\int_{S} \omega \cdot \mathbf{n} d S=0
$$

donde $S$ es la superficie que limita el volumen $V$ con unidad normal $\mathbf{n}$ externa. Por tanto, el flujo de vorticidad a través de una superficie cerrada es cero. La misma afirmación es válida para el flujo de la velocidad.

El flujo se dice que es irrotacional si $\nabla \times \mathbf{u}=0$, es decir si la vorticidad es cero. En este caso existe una función escalar $\phi$, llamada el potencial de velocidad, tal que $\mathbf{u}=\nabla \phi$ siempre y cuando el flujo es en todo $\mathbb{R}^{3}$ o en una región simplemente conexa. Esto se sigue de (3.2) ya que $\phi$ satisface la ecuación de Laplace $\nabla^{2} \phi=0$ y escribimos $\mathbf{u}=\mathbf{u}_{\phi}$ para el potencial de flujo. En general, el campo de velocidad puede ser descompuesto en términos del potencial de velocidad, más un vector potencial solenoidal $\psi$ (algunas veces referida como descomposición de Helmholtz-Hodge), donde $\nabla \cdot \psi=0$ $\mathrm{y}$

$$
\mathbf{u} \equiv \mathbf{u}_{\phi}+\mathbf{u}_{\omega}=\nabla \phi+(\nabla \times \psi)
$$

El segundo término es generado por la distribución de vorticidad.

De la ecuación (3.1) y de $\mathbf{u}_{\omega}=\nabla \times \psi$ tenemos que

$$
\begin{aligned}
\omega & =\nabla \times(\nabla \times \psi) \\
& =\nabla(\nabla \cdot \psi)-\nabla^{2} \psi \\
& =-\nabla^{2} \psi
\end{aligned}
$$

y obtenemos

$$
\nabla^{2} \psi=-\omega
$$

Es decir, el vector potencial satisface la ecuación de Poisson con vorticidad sobre el lado derecho. Por técnicas estándar uno puede escribir la solución de 
la ecuación de Poisson en término de la función de Green para el Laplaciano (en el espacio libre)

$$
\psi(\mathbf{x})=\int G(\mathbf{x}-\mathbf{z}) \omega(\mathbf{z}) d z
$$

donde

$$
G(\mathbf{x})=\left\{\begin{array}{cc}
-\frac{1}{2 \pi} \log \|\mathbf{x}\| & \text { en } \mathbb{R}^{2} \\
\frac{1}{4 \pi} \frac{1}{\|\mathbf{x}\|} & \text { en } \mathbb{R}^{3}
\end{array}\right.
$$

con

$$
\nabla^{2} G(\mathbf{x})+\delta(\mathbf{x})=0 .
$$

Entonces, dado que $\mathbf{u}_{\omega}(\mathbf{x})=\nabla \times \psi$, tenemos

$$
\begin{aligned}
\mathbf{u}_{\omega}(\mathbf{x}) & =\nabla \times \int G(\mathbf{x}-\mathbf{z}) \omega(\mathbf{z}) d \mathbf{z} \\
& =\int K(\mathbf{x}-\mathbf{z}) \omega(\mathbf{z}) d \mathbf{z}
\end{aligned}
$$

donde $K$ es el kernel singular Biot-Savart definido como

$$
K(\mathbf{x})=\left\{\begin{array}{cc}
\frac{1}{2 \pi} \frac{1}{\|\mathbf{x}\|^{2}}(-y, x) & \text { en } \mathbb{R}^{2} \\
\frac{1}{4 \pi} \frac{1}{\|\mathbf{x}\|^{3}}\left(\begin{array}{ccc}
0 & z & -y \\
-z & 0 & x \\
y & -x & 0
\end{array}\right) & \text { en } \mathbb{R}^{3}
\end{array}\right.
$$

A menudo la fórmula de Biot-Savart en $\mathbb{R}^{3}$ se escribe:

$$
\mathbf{u}_{\omega}(\mathbf{x})=-\frac{1}{4 \pi} \int \frac{(\mathbf{x}-\mathbf{z}) \times \omega(\mathbf{z}) d \mathbf{z})}{\|\mathbf{x}-\mathbf{z}\|^{3}} .
$$

Una cantidad escalar fundamental asociada con la vorticidad es la circulación $\Gamma=\oint \mathbf{u} \cdot d \mathbf{s}$ de un fluido alrededor de una curva simple cerrada. El teorema de Stokes nos dice

$$
\Gamma=\oint_{C} \mathbf{u} \cdot d \mathbf{s}=\int_{A} \omega \cdot \mathbf{n} d \mathbf{s}
$$

donde $A$ es cualquier superficie abierta acotada por la curva cerrada $C$. Esta fórmula muestra que la circulación es el flujo de vorticidad a través de una superficie abierta $A$ acotada por la curva. El resultado principal concerniente a circulación es el teorema de circulación de Kelvin, el cual dice que 
en un fluido ideal incompresible, actuando por fuerzas conservativas, la circulación $\Gamma(t)$ moviéndose con el fluido es constante i.e. $\frac{d \Gamma}{d t}=0$ (ver [8] o [36] para las demostraciones). La vorticidad no puede ser creada a menos que una de las condiciones del teorema sea violada, por ejemplo, la introducción de capas viscosas o la presencia de estratificación. Ambas son efectos físicos discutidos extensamente en la literatura. Si la circulación es cero para todas las curvas cerradas, entonces el flujo es irrotacional. Lo contrario es cierto si el fluido está contenido en una región simplemente conexa.

Las curvas trazadas paralelas al vector local de vorticidad en cada punto son llamadas líneas de vorticidad, mientras que el conjunto de líneas de vórtices que pasan por los puntos de un curva cerrada en el flujo se llaman tubo de vórtice. Por construcción, un tubo de vórtice tiene la propiedad de que la vorticidad es siempre paralela a la superficie del mismo, es decir, $\omega \cdot \mathbf{n}=0$ sobre la superficie de un tubo de vórtice, por lo tanto el flujo de vorticidad a través de cualquier sección transversal del tubo es una constante. El flujo de vorticidad a lo largo del tubo es igual a la circulación alrededor de cualquier curva cerrada sobre la pared del tubo rodeando el tubo una vez, que se llama la fuerza tubo. Un tubo de vórtice que está rodeado por el fluido irrotacional se llama un filamento de vórtice. Típicamente, un filamento de vórtice tiene una sección transversal pequeña en comparación con su curvatura. El hecho de que la circulación no cambia a lo largo de un tubo significa que los tubos de vórtice son cerrados, se van hasta el infinito, o terminan en las fronteras del sólido o interfaces líquido-líquido. (ver [40] y [27], 28] para una discusión de la geometría de tubo de vórtice.)

El flujo ideal incompresible en tres dimensiones actuado por fuerzas conservativas, tiene que sus ecuaciones de movimiento básicas basadas en las leyes de Newton $\mathbf{F}=m \mathbf{a}$ están dadas por las ecuaciones incompresibles de Euler

$$
\frac{D \mathbf{u}}{D t} \equiv \mathbf{u}_{t}+\mathbf{u} \cdot \nabla \mathbf{u}=\nabla p+\mathbf{f}
$$

donde $\mathbf{u} \in \mathbb{R}^{3}$ y $\frac{D}{D t} \equiv\left(\frac{\partial}{\partial t}+\mathbf{u} \cdot \nabla\right)$ es el operador derivada material, $p$ es el campo de presión y f representa las fuerzas externas del cuerpo por unidad de masa.

Para flujos no viscosos las condiciones de frontera están dadas por $\mathbf{u} \cdot \mathbf{n}=0$ sobre la frontera.

Tomemos el rotacional de 3.14), es decir 


$$
\nabla \times \frac{D \mathbf{u}}{D t}=\nabla \times\left(\mathbf{u}_{t}+\mathbf{u} \cdot \nabla \mathbf{u}\right)=-(\nabla \times \nabla p)+(\nabla \times \mathbf{f}) .
$$

Como sabemos, el rotacional de un gradiente de cualquier campo escalar es cero, es decir

$$
-(\nabla \times \nabla p)=0
$$

y también sabemos que $\mathbf{f}$ es conservativo, por lo tanto

$$
\nabla \times \mathbf{f}=0
$$

entonces tenemos lo siguiente

$$
\nabla \times\left(\mathbf{u}_{t}+\mathbf{u} \cdot \nabla \mathbf{u}\right)=0 .
$$

Ahora veamos quien es el lado izquierdo de la ecuación.

$$
\begin{aligned}
\nabla \times\left(\mathbf{u}_{t}+\mathbf{u} \cdot \nabla \mathbf{u}\right) & =\left(\nabla \times \mathbf{u}_{t}\right)+(\nabla \times(\mathbf{u} \cdot \nabla \mathbf{u})) \\
& =\frac{\partial}{\partial t}(\nabla \times \mathbf{u})+\left(\nabla \times\left(\frac{1}{2} \nabla(\mathbf{u} \cdot \mathbf{u})-(\mathbf{u} \times \omega)\right)\right) \\
& =\frac{\partial}{\partial t}(\nabla \times \mathbf{u})+\frac{1}{2}(\nabla \times \nabla(\mathbf{u} \cdot \mathbf{u}))-(\nabla \times(\mathbf{u} \times \omega)) \\
& =\frac{\partial}{\partial t}(\nabla \times \mathbf{u})-\mathbf{u}(\nabla \cdot \omega)+\omega(\nabla \cdot \mathbf{u})-(\omega \cdot \nabla) \mathbf{u}+(\mathbf{u} \cdot \nabla) \omega
\end{aligned}
$$

por tanto tenemos

$$
\begin{aligned}
\frac{\partial}{\partial t}(\nabla \times \mathbf{u})-(\omega \cdot \nabla) \mathbf{u}+(\mathbf{u} \cdot \nabla) \omega & =0 \\
\frac{\partial}{\partial t}(\nabla \times \mathbf{u})+(\mathbf{u} \cdot \nabla) \omega & =(\omega \cdot \nabla) \mathbf{u} \\
\frac{D \omega}{D t} & =\omega \cdot \nabla \mathbf{u}
\end{aligned}
$$

y llegamos a la ecuación de evolución de la vorticidad

$$
\frac{D \omega}{D t}=\omega \cdot \nabla \mathbf{u}
$$

La ventaja de esta formulación es la ausencia del término de presión en las ecuaciones (la evolución de vorticidad depende sólo de los valores locales y vorticidad del fluido). El término de la derecha en 3.15 es comúnmente 
conocido como el vórtice de estiramiento, que es muy importante para muchos aspectos tres-dimensionales de flujos turbulentos. Notemos también que si $\omega(\mathbf{x}, 0)=0$, entonces $\omega(\mathbf{x}, t)=0$ para $t>0$, que significa que si la vorticidad es cero inicialmente, ésta se mantiene cero para todo tiempo.

Si una partícula pasiva se coloca en el campo de flujo en la posición $\mathbf{x}(t)$, se ve afectada por el campo de velocidades de acuerdo a la ecuación

$$
\dot{\mathbf{x}}=\mathbf{u}(\mathbf{x}, t)
$$

Ahora, si diferenciamos esta ecuación, uno puede ver que en un elemento de línea infinitesimal $\delta \boldsymbol{l}$ satisface la ecuación de evolución (ver [4] para la demostración), es decir tenemos

$$
\frac{D \delta \boldsymbol{l}}{D t}=\delta \boldsymbol{l} \cdot \nabla \mathbf{u}
$$

Dado que esta ecuación es idéntica a la ecuación de vorticidad 3.15 con $\delta \boldsymbol{l}$ y $\omega$ intercambiados, podemos concluir que las líneas de vórtice se mueven precisamente como lo hacen las líneas de materiales. En particular, el lado derecho de la ecuación muestra cómo en tres dimensiones, las líneas de material se estiran o se comprimen por el flujo.

Ahora bien, para fluidos viscosos existe un término adicional en el lado derecho de la ecuación (3.15) causando difusión de vorticidad dada por $R^{-1} \nabla^{2} \omega$, donde $R$ es el número de Reynolds $R=\frac{D}{\rho v^{2}}$. En este caso, el teorema de circulación de Kelvin se transforma en

$$
\frac{d \Gamma}{d t}=-R^{-1} \oint(\nabla \times \omega) \cdot d S
$$

mostrando que la circulación ya no es constante. Para los campos de flujo donde la vorticidad está confinada a una superficie dos dimensional, el vector de vorticidad tiene una sola componente en la dirección perpendicular a la superficie, de ahí el vórtice de estiramiento está ausente. Principalmente por esta razón, los flujos dos-dimensionales son diferentes de los flujos tresdimensionales. De hecho, la teoría básica de la existencia de las ecuaciones de Euler en $\mathbb{R}^{3}$ (para tiempo largo) aún no ha sido resuelta (ver [5] [11], [18], [33, [38, [39]). Se cree por algunos que el término de vórtice de estiramiento produce una singularidad de tiempo finito (ver, por ejemplo, [13, [19], [31], [34, [35]) y sabemos que la clave para esto es la cantidad $\int_{0}^{T}\|\omega\|_{L^{\infty}} d t$, donde $\|\cdot\|_{L^{\infty}}$ es la norma $L^{\infty}$, que debe estallar si no es una singularidad 
de tiempo-finito, como muestra [5] y se discute más a detalle en los libros de [10], [21] y [23]. En $\mathbb{R}^{2}$ cuando el término de vórtice de estiramiento está ausente, la existencia a largo tiempo está establecida; ver [3], [17], 42] y [43].

\subsection{Invariantes de las ecuaciones de Euler}

Las ecuaciones sin viscosidad tienen varias invariantes que desempeñaran un papel importante. Asumiendo que el campo de velocidades decae suficientemente rápido en el infinito y que no hay fuerzas externas del cuerpo, el total de la energía cinética $E$ en el flujo y la vorticidad total $W_{1}$ son constantes

$$
\begin{aligned}
E(t) & =\frac{1}{2} \int_{\mathbb{R}^{3}}\|\mathbf{u}\|^{2} d \mathbf{x}=E(0) \\
W_{1}(t) & =\int_{\mathbb{R}^{3}} \omega(\mathbf{x}) d \mathbf{x}=W_{1}(0)
\end{aligned}
$$

Para flujos viscosos, la energía cinética ya no es constante ya que la viscosidad hace que se transforme en energía interna. En general, tenemos

$$
\frac{d E}{d t}=-R^{-1} \int_{\mathbb{R}^{3}}\|\omega\|^{2} d \mathbf{x} \leq 0 .
$$

Sin embargo, dado que las ecuaciones macroscópicas no incluyen efectos microscópicos, no hay conteo de aumento en la energía interior para equilibrar la disminución de energía cinética.

Las ecuaciones de Euler son invariantes con respecto a traslaciones espaciales y rotaciones. Por lo tanto "momento lineal" L y el "momento angular" $I$, de el fluido son constantes,

$$
\begin{aligned}
L(t) & =\frac{1}{2} \int_{\mathbb{R}^{3}}(\mathbf{x} \times \omega) d \mathbf{x}=L(0) \\
I(t) & =\frac{1}{3} \int_{\mathbb{R}^{3}} \mathbf{x} \times(\mathbf{x} \times \omega) d \mathbf{x} \\
& =-\frac{1}{2} \int_{\mathbb{R}^{3}}\|\mathbf{x}\|^{2} \omega d \mathbf{x}=I(0) .
\end{aligned}
$$

Estás cantidades también son constantes para flujos viscosos. Un interesante invariante topológico para los flujos sin viscosidad se muestra en [27], es la llamada helicidad, $J$ donde

$$
J=\int_{\mathbb{R}^{3}} \mathbf{u} \cdot \omega d \mathbf{x}=J(0)
$$


Esta conservación se sigue esencialmente del teorema de circulación de Kelvin. La helicidad mide el grado de enlace de grupos de filamentos de vórtices cerrados. Por ejemplo si nosotros tenemos una colección de $n$ enlaces de filamentos de vórtices $C_{k}$, donde $k=1, \cdots, n$, cada uno de fuerza $\Gamma_{k}$ entonces

$$
J=\sum_{i, j} \alpha_{i j} \Gamma_{i} \Gamma_{j}
$$

donde $\alpha_{i j}$ es el número de vueltas de $C_{i}$ y $C_{j}$ (i.e. el número entero de veces donde los filamentos se enredan alrededor del otro). Esto se ve asumiendo que la distribución de vorticidad $\omega$ es cero, excepto en 2 (ó más) filamentos cerrados $C_{1}, C_{2}$. Entonces la integral de volumen definiendo $J$, degenera a

$$
J=\Gamma_{1} \oint_{C_{1}} \mathbf{u} \cdot d \mathbf{x}+\Gamma_{2} \oint_{C_{2}} \mathbf{u} \cdot d \mathbf{x} .
$$

Por el teorema de Stokes, la primera integral es igual al flujo de vorticidad a través de la superficie que abarca $C_{1}$, que está dada por $\pm n \Gamma_{2}$, es decir se ve como sigue

$$
\begin{aligned}
\Gamma_{1} \oint_{C_{1}} \mathbf{u} \cdot d \mathbf{x} & =\Gamma_{1} \int_{S}(\nabla \times \mathbf{u}) \cdot d \mathbf{s} \\
& =\Gamma_{1} \int_{S} \omega \cdot d \mathbf{s} \\
& = \pm n \Gamma_{1} \Gamma_{2}
\end{aligned}
$$

donde $n$ es el número de enlace, (número de veces que $C_{2}$ perfora la superficie de expansión de $C_{1}$ ) y \pm es escogido si el enlace es derecho (ó izquierdo). Se argumenta de manera similar para la segunda integral, obteniendo

$$
J= \pm n \Gamma_{1} \Gamma_{2} \pm n \Gamma_{2} \Gamma_{1}= \pm m \Gamma_{1} \Gamma_{2}
$$

Este resultado se puede generalizar de forma obvia para muchos filamentos.

Sin embargo, el campo de vorticidad no puede ser explícitamente escrito como una colección de enlaces de tubos de vórtice. Pero uno puede siempre descomponer $\omega$ en la suma de tres campos

$$
\omega \equiv \omega_{1}+\omega_{2}+\omega_{3}
$$

donde

$$
\omega_{1}=\left(0, \frac{\partial u_{1}}{\partial z},-\frac{\partial u_{1}}{\partial y}\right)
$$




$$
\begin{aligned}
\omega_{2} & =\left(-\frac{\partial u_{2}}{\partial z}, 0, \frac{\partial u_{2}}{\partial x}\right) \\
\omega_{1} & =\left(\frac{\partial u_{3}}{\partial y},-\frac{\partial u_{3}}{\partial x}, 0\right) .
\end{aligned}
$$

Dado que las líneas de vórtice para el campo $\omega_{1}$ son curvas cerradas dadas por $u_{1}=$ const., $x=$ const. la misma helicidad es cero. Esto es cierto para $\omega_{2}$ y $\omega_{3}$ y la invarianza de la helicidad puede ser escrita

$$
J=\sum_{n \neq m} J_{n m}, \quad J_{n m}=\int \mathbf{u}_{n} \cdot \omega_{m} d V
$$

donde $J_{n m}$ es el grado de enlace de los campos $\omega_{n}, \omega_{m}$. Ver [29] para una mayor discusión.

Aunque en tres dimensiones no se conserva, una importante cantidad es la enstrofía $W_{2}$

$$
W_{2}=\frac{1}{2} \int_{\mathbb{R}^{3}}\|\omega\|^{2} d \mathbf{x}
$$

Como hemos visto, la energía y la enstrofía están relacionadas por

$$
\frac{d E}{d t}=-2 R^{-1} W_{2}
$$

Otra cantidad común es la palinstropía $P$

$$
P=\frac{1}{2} \int_{\mathbb{R}^{3}}(\nabla \times \omega)^{2} d \mathbf{x}
$$

que pueden ser relacionadas por el radio de cambio de la enstrofía vía

$$
\frac{d W_{2}}{d t}=-2 R^{-1} P+\int_{\mathbb{R}^{3}} \omega \cdot(\omega \cdot \nabla) \mathbf{u} d \mathbf{x} .
$$

La helicidad, la energía y la enstrofía de el flujo están relacionadas vía la desigualdad de Schwarz para dar una cota inferior en la enstrofía

$$
\begin{aligned}
|J| & =\left|\int_{\mathbb{R}^{3}}\langle\mathbf{u}, \omega\rangle d \mathbf{x}\right| \\
& \leq\left|\int_{\mathbb{R}^{3}}\langle\mathbf{u}, \mathbf{u}\rangle d \mathbf{x}\right|\left|\int_{\mathbb{R}^{3}}\langle\omega, \omega\rangle d \mathbf{x}\right| \\
& =|E|\left|W_{2}\right| \\
|J| & \leq|E|\left|W_{2}\right|
\end{aligned}
$$


de donde tenemos que

$$
W_{2} \geq \frac{4 J}{E}
$$

ver [1] [27] y 29] para una mayor discusión de esto.

\subsection{Vorticidad en dos dimensiones}

Ahora nos enfocaremos en dos dimensiones, tenemos que el lado derecho de (3.15) es cero, es decir, consideramos $\mathbf{u}=(u, v, 0)$ de donde tenemos

$$
\begin{aligned}
\frac{D \omega}{D t} & =\omega \cdot \nabla \mathbf{u} \\
& =(\nabla \times \mathbf{u}) \cdot \nabla \mathbf{u} \\
& =0
\end{aligned}
$$

por lo cual la ecuación de la evolución de la vorticidad se convierte en una ley de conservación para la vorticidad escalar

$$
\frac{D \omega}{D t} \equiv 0
$$

donde $\omega=\frac{\partial v}{\partial x}-\frac{\partial u}{\partial y}$ Para el flujo 2-dimensional, existe una jerarquía infinita de cantidades invariantes $W_{k}(k=1,2,3, \ldots)$, llamada momento de vorticidad, donde

$$
W_{k}=\frac{1}{k} \int_{\mathbb{R}^{2}} \omega^{k}(\mathbf{x}) d \mathbf{x}
$$

Así, en dos dimensiones tanto la energía como la enstrofía son cantidades conservadas (un hecho que usualmente se dice que prohíbe una cascada de energía a pequeña escala), mientras que en 3 dimensiones la enstrofía puede incrementar, debido a que el tubo de vórtice se extiende, como es discutido en [20]. De hecho se puede verificar que para cualquier función suave de la vorticidad $f(\omega)$ es constante bajo el flujo. Para ver esto consideremos un camino cerrado $C$, encerrado en el área $A$ que se mueve con el campo de flujo. Entonces

$$
\frac{d}{d t} \int_{C} f(\omega(\mathbf{x})) d \mathbf{x}=\int_{C} f^{\prime}(\omega(\mathbf{x}))\left[\frac{d \omega}{d t}+\mathbf{u} \cdot \nabla \omega\right] d \mathbf{x}=0
$$

Una característica adicional de flujos 2-dimensionales no disponible para flujos 3-dimensionales (a menos que estos posean cierta simetría; ver los 
artículos [15] y [26] para mayor información) es que vector potencial $\psi$ se convierte en la función escalar, llamada la función de corriente. Así

$$
\begin{aligned}
\mathbf{u} & =\nabla \times \psi \\
& =\nabla^{\perp} \psi \\
& \equiv\left(\begin{array}{cc}
0 & 1 \\
-1 & 0
\end{array}\right)\left(\begin{array}{c}
\partial_{x} \\
\partial_{y}
\end{array}\right) \psi \\
& =\left(\psi_{y},-\psi_{x}\right) .
\end{aligned}
$$

La ecuación para una partícula de fluido se convierte en

$$
\begin{aligned}
\dot{\mathbf{x}} & =\mathbf{u} \\
& =\left(\psi_{y},-\psi_{x}\right)
\end{aligned}
$$

que toman la forma de las ecuaciones canónicas de Hamilton

$$
\begin{aligned}
\dot{x} & =\frac{\partial \mathcal{H}}{\partial y}(x, y ; t) \\
\dot{y} & =-\frac{\partial \mathcal{H}}{\partial x}(x, y ; t)
\end{aligned}
$$

El Hamiltoniano (dependiente del tiempo) juega el papel de la función de corriente $\mathcal{H} \equiv \psi$, con las coordenadas de posición $(x, y)$ haciendo el papel de variables canónicas conjugadas. Cuando el flujo es independiente del tiempo, las curvas de nivel de las líneas de corriente Hamiltoniana que las partículas de fluido (también llamadas "marcador pasivas") siguen, tienen la propiedad de que su velocidad es tangente a las líneas de corriente. Esto puede ser entendido, observando que el vector normal $\mathbf{n}$ a la curva de nivel $\psi=$ const. esta dado por

$$
\mathbf{n}=\nabla \psi=\nabla \mathcal{H}
$$

Dado que $\mathbf{u} \cdot \mathbf{n}=\mathbf{u} \cdot \nabla \psi=0$, la velocidad local del fluido es tangente a cada curva de nivel. También, dado que $\mathbf{u} \cdot \mathbf{n}=0$ cada línea de corriente puede ser vista como un sólido acotado, que ningún fluido puede penetrar.

Ahora mencionaremos que en dos dimensiones, y asumiendo que la función de corriente $\psi$ es armónica (por lo que $\omega=-\nabla^{2} \psi=0$ ) uno puede definir una cantidad compleja llamada el potencial complejo.

Definición 3.3.1. La función analítica compleja

$$
w(z) \equiv \phi+i \psi
$$


es llamada el potencial complejo para el flujo, donde $z=x+i y$. La parte real, $\phi$, es el potencial de velocidad, mientras la parte imaginaria, $\psi$, es la función de corriente. Dado que la función $w(z)$ es analítica, $\phi$ y $\psi$ están relacionadas mediante las ecuaciones de Cauchy-Riemann

$$
\begin{aligned}
& \frac{\partial \phi}{\partial x}=\frac{\partial \psi}{\partial y} \\
& \frac{\partial \phi}{\partial y}=-\frac{\partial \psi}{\partial x}
\end{aligned}
$$

La velocidad compleja esta definida por

$$
\frac{d w}{d z}=u-i v
$$

Ocasionalmente, es útil para escribir la velocidad y vorticidad en términos de coordenadas polares. Tenemos lo siguiente

$$
\begin{aligned}
\left(\begin{array}{l}
u \\
v
\end{array}\right) & =\frac{1}{r}\left(\begin{array}{cc}
r \cos \theta & -r \operatorname{sen} \theta \\
r \operatorname{sen} \theta & r \cos \theta
\end{array}\right)\left(\begin{array}{l}
u_{r} \\
u_{\theta}
\end{array}\right) \\
& =\left(\begin{array}{cc}
\cos \theta & -\operatorname{sen} \theta \\
\operatorname{sen} \theta & \cos \theta
\end{array}\right)\left(\begin{array}{l}
u_{r} \\
u_{\theta}
\end{array}\right)
\end{aligned}
$$

y podemos escribir entonces $u+i v=e^{i \theta}\left(u_{r}+i u_{\theta}\right)$, de donde tenemos que

$$
\begin{aligned}
\frac{\partial w}{\partial z} & =\overline{u+i v} \\
& =e^{-i \theta}\left(u_{r}-i u_{\theta}\right) .
\end{aligned}
$$

Por otra parte tenemos

$$
\begin{aligned}
\frac{\partial \phi}{\partial x}+i \frac{\partial \phi}{\partial y} & =\frac{\partial \phi}{\partial x}-i \frac{\partial \psi}{\partial x} \\
\frac{\partial \phi}{\partial x}-i \frac{\partial \psi}{\partial x} & =e^{i \theta}\left(u_{r}-i u_{\theta}\right) \\
& =(\cos \theta-i \operatorname{sen} \theta)\left(u_{r}-i u_{\theta}\right) \\
& =u_{r} \cos \theta-u_{\theta} \operatorname{sen} \theta+i\left(-u_{r} \operatorname{sen} \theta-u_{\theta} \cos \theta\right)
\end{aligned}
$$

y también que

$$
\frac{d w}{d z}=\cos \theta \frac{1}{r} \frac{\partial \psi}{\partial \theta}+\operatorname{sen} \theta \frac{\partial \psi}{\partial r}+i\left(\cos \theta \frac{\partial \psi}{\partial r}-\frac{\operatorname{sen} \theta}{r} \frac{\partial \psi}{\partial \theta}\right)
$$


entonces,

$$
\begin{aligned}
-u_{r} \operatorname{sen} \theta-u_{\theta} \cos \theta & =\cos \theta \frac{\partial \psi}{\partial r}-\frac{\operatorname{sen} \theta}{r} \frac{\partial \psi}{\partial \theta} \\
\cos \theta\left(\frac{\partial \psi}{\partial r}+u_{\theta}\right)+\operatorname{sen}\left(-\frac{1}{r} \frac{\partial \psi}{\partial \theta}+u_{r}\right) & =0 .
\end{aligned}
$$

Por tanto, las componentes de la velocidad radial y azimutal $\left(u_{r}, u_{\theta}\right)$ están dadas por

$$
u_{r}=\frac{1}{r} \frac{\partial \psi}{\partial \theta}, \quad u_{\theta}=-\frac{\partial \psi}{\partial r} .
$$

Ahora, considerando el rotacional en coordenadas cilíndricas, tenemos que

$$
\begin{aligned}
\nabla \times \mathbf{u} & =\left(\frac{1}{r} \frac{\partial u_{z}}{\partial \theta}-\frac{\partial u_{\theta}}{\partial z}\right) \widehat{\mathbf{r}}+\left(\frac{\partial u_{r}}{\partial z}-\frac{\partial u_{z}}{\partial r}\right) \widehat{\theta}+\frac{1}{r}\left(\frac{\partial\left(r u_{\theta}\right)}{\partial r}-\frac{\partial u_{r}}{\partial \theta}\right) \widehat{\mathbf{z}} \\
& =\frac{1}{r}\left(\frac{\partial\left(r u_{\theta}\right)}{\partial r}-\frac{\partial u_{r}}{\partial \theta}\right) \widehat{\mathbf{z}}
\end{aligned}
$$

y entonces obtenemos que la vorticidad está dada por

$$
\omega=-\frac{1}{r} \frac{\partial}{\partial r}\left(r \frac{\partial \psi}{\partial r}\right)-\frac{1}{r^{2}} \frac{\partial^{2} \psi}{\partial \theta^{2}} .
$$

Para ilustrar la elegancia del uso del potencial complejo listaremos algunos ejemplos:

\section{Flujo de punto fuente}

Tenemos que el potencial complejo, la velocidad potencial, la función de corriente y la vorticidad están dadas por las siguientes ecuaciones

$$
\begin{aligned}
w(z) & =\frac{m}{2 \pi} \log \left(z-z_{0}\right) \\
\phi(z) & =\frac{m}{2 \pi} \log \left|z-z_{0}\right| \\
\psi(z) & =\frac{m}{2 \pi} \arg \left(z-z_{0}\right) \\
\frac{d w}{d z} & =\frac{m}{2 \pi} \cdot \frac{\left(z-z_{0}\right)^{*}}{\left|z-z_{0}\right|^{2}} \\
\omega & =0
\end{aligned}
$$




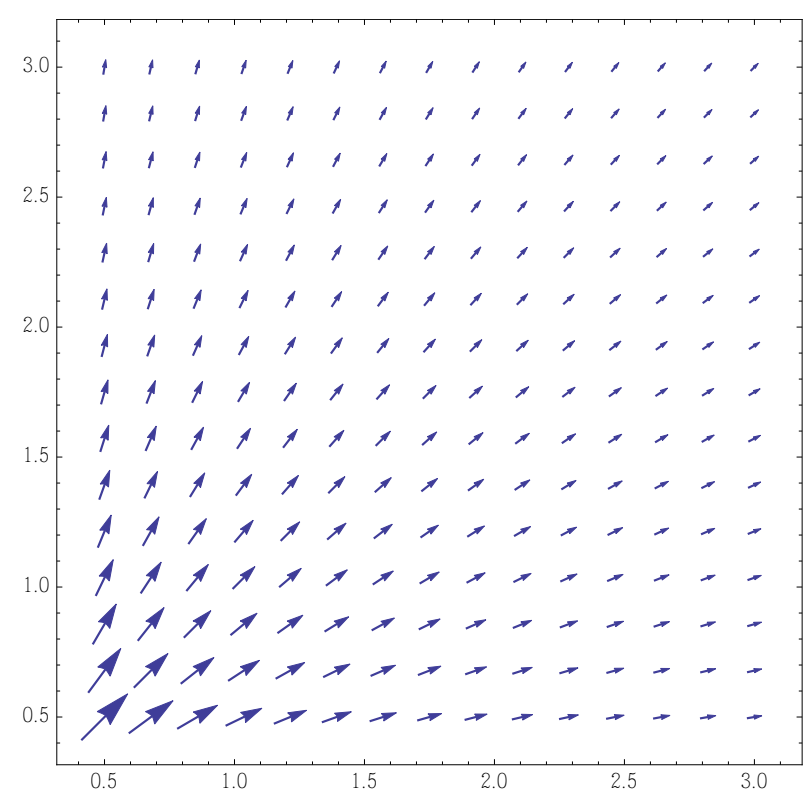

Figura 3.1: Campo de velocidad del flujo de un punto fuente.

Poniendo $z_{0}$ en el origen y haciendo $m=2 \pi$, la velocidad compleja es

$$
\left(\frac{d w}{d z}\right)^{*}=1 / \bar{z}=\frac{x+i y}{x^{2}+y^{2}} .
$$

El correspondiente flujo de punto fuente se ilustra en la figura 3.1. (Solo mostramos el primer cuadrante; el flujo sobre los otros tres cuadrantes se obtiene por simetría reflejando con respecto a los ejes cartesianos.)

\section{Flujo de un vórtice puntual}

$$
\begin{aligned}
w(z) & =\frac{\Gamma}{2 \pi i} \log \left(z-z_{0}\right) \\
\phi(z) & =\frac{\Gamma}{2 \pi} \arg \left(z-z_{0}\right) \\
\psi(z) & =-\frac{\Gamma}{2 \pi} \cdot \log \left|z-z_{0}\right| \\
\frac{d w}{d z} & =\frac{\Gamma}{2 \pi i} \cdot \frac{\left(z-z_{0}\right)^{*}}{\left|z-z_{0}\right|^{2}} \\
\omega & =0
\end{aligned}
$$




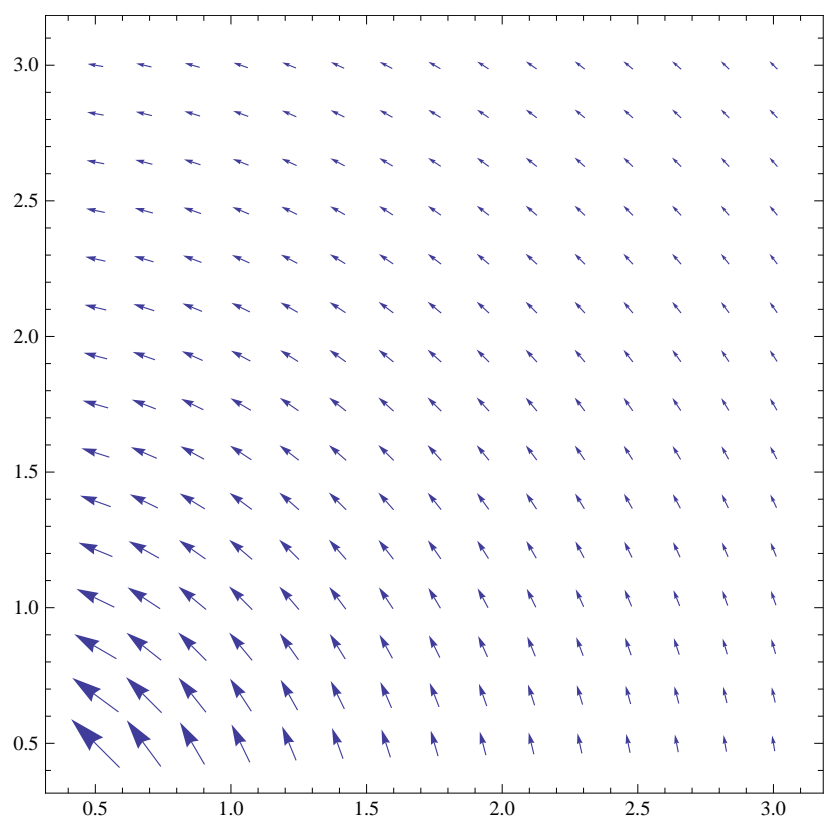

Figura 3.2: Campo de velocidad del flujo inducido por un vórtice puntual. Se muestra una región en el primer cuadrante que excluye el origen (donde la velocidad se hace infinita).

El flujo debido a un vórtice puntual se ilustra en la figura 3.2

3. Flujo de un punto esquina, con ángulo $\pi / m$

$$
\begin{aligned}
w(z) & =A z^{m} \\
\phi(z) & =A r^{m} \cos (m \theta) \\
\psi(z) & =A r^{m} \operatorname{sen}(m \theta) \\
\frac{d w}{d z} & =m A z^{m-1} \\
\omega & =0
\end{aligned}
$$

El flujo de un punto esquina se ilustra en la figura 3.3 para tres valores distintos del ángulo.

Procedimiento para obtener las gráficas de flujo. En los tres ejemplos anteriores, el procedimiento para obtener las gráficas de flujo y las expresiones 

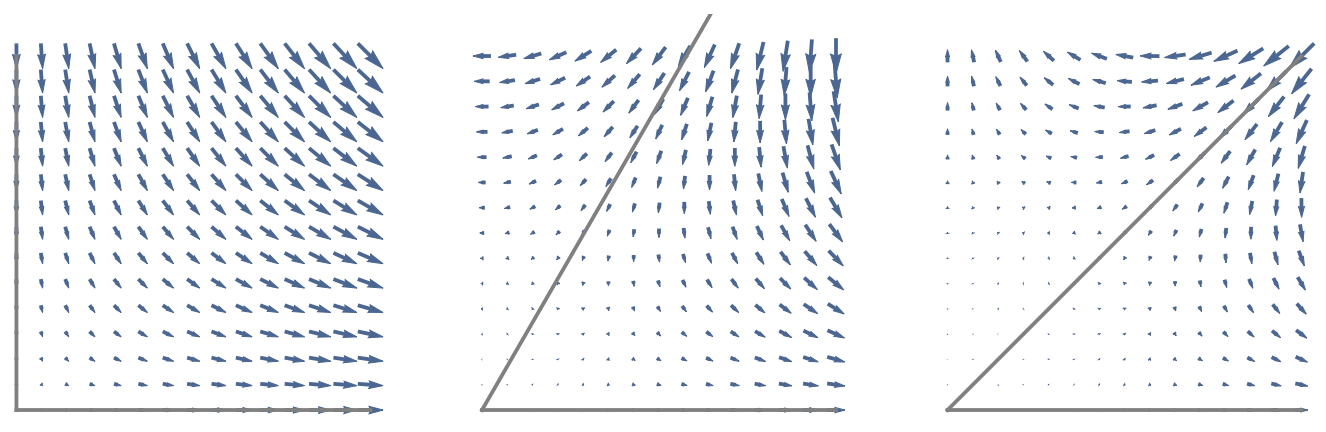

Figura 3.3: Campo de velocidad para el flujo de una esquina con ángulo $90^{\circ}$, $60^{\circ}$ y $45^{\circ}$.

correspondientes es el siguiente: a) Dar la función $\phi$ cuyas curvas de nivel corresponden a las líneas de flujo que se desea estudiar; esta función debe ser armónica. b) Dar la función armónica conjugada $\psi$, formando la función compleja $w=\phi+i \psi$. c) El campo de velocidad del fluido está dado, en notación compleja, por $(d w / d z)^{*}$. (El asterisco denota tomar el conjugado.)

\subsection{Descomposición en vórtices puntuales}

Pasemos al caso de delgados filamentos de vorticidad paralelos y formulemos las ecuaciones para la dinámica de un punto de vórtice en una superficie de dos dimensiones. Cuando la vorticidad está concentrada perfectamente y a lo largo de un filamento recto de vorticidad, un corte de este filamento por una superficie dos-dimensional nos da una distribución de vorticidad en el plano que está altamente localizada alrededor de la línea central del filamento, es decir, la función de vorticidad tiene un pequeño soporte. En general, para un representante discreto de vórtice en dos dimensiones, se supone una distribución de vorticidad en la forma

$$
\begin{aligned}
\omega(\mathbf{x}) & =\sum_{i=1}^{N} \frac{\Gamma_{i}}{2 \pi} \phi_{\epsilon}\left(\mathbf{x}-\mathbf{x}_{i}\right) \\
\phi_{\epsilon}(\mathbf{x}) & =\frac{1}{\epsilon^{2}} \phi\left(\frac{\mathbf{x}}{\epsilon}\right)
\end{aligned}
$$


con $\epsilon<<1$ y $\phi$ cualquier función adecuadamente normalizada, radialmente simétrica de manera que $\int \phi d \mathbf{x}=2 \pi$. El caso límite $(\epsilon \rightarrow 0)$ de dicha representación, llamado un vórtice puntual, está dado por la elección

$$
\phi_{\epsilon}(\mathbf{x})=\delta(\mathbf{x})
$$

donde $\delta$ es la delta de Dirac; en la sección 2.4 hemos recordado cómo se define la Delta de Dirac. El campo de velocidad y vorticidad asociado con un punto aislado del vórtice está dado por

$$
\begin{aligned}
u_{\theta}(r, \theta, z) & =\frac{\Gamma}{2 \pi r} \\
u_{r} & =0 \\
u_{z} & =0 \\
\omega_{z} & =\delta(r)
\end{aligned}
$$

Este flujo es algunas veces referido como vórtice irrotacional dado que $\omega=0$, excepto en la localización del vórtice puntual, el cual tiene medida cero. Físicamente, la aproximación discreta del vórtice es análoga a la aproximación "masa-punto" usada en problemas gravitacionales de $N$-cuerpos, donde la estructura interna y deformación de la vorticidad local es ignorada y uno sólo está interesado en la búsqueda de la vorticidad máxima o punto central de la región de vorticidad.

Para obtener las ecuaciones para la dinámica de una colección de $N$ vórtices localizados en $\mathbf{x}_{\alpha}=\left(x_{\alpha}(t), y_{\alpha}(t)\right)$ donde $\alpha=1, \ldots, N$, comenzamos con las ecuaciones de velocidad y notemos que la velocidad inducida por un punto aislado del vórtice es

$$
\begin{aligned}
\dot{\mathbf{x}_{\alpha}} & =\mathbf{u}\left(\mathbf{x}_{\alpha}, t\right) \\
& =\nabla^{\perp} \psi_{\alpha}\left(\mathbf{x}_{\alpha}, t\right) .
\end{aligned}
$$

De (3.8) vemos que

$$
\begin{aligned}
\psi_{\alpha}(\mathbf{x}, t) & =-\frac{1}{2 \pi} \int \Gamma_{\alpha} \log |\mathbf{x}-\mathbf{z}| \delta\left(\mathbf{x}_{\alpha}-\mathbf{z}\right) d \mathbf{z} \\
& =-\frac{\Gamma_{\alpha}}{2 \pi} \log \left|\mathbf{x}-\mathbf{x}_{\alpha}\right| .
\end{aligned}
$$

El campo de velocidad debida a una colección de $N$ vórtices es obtenida por una superposición lineal (dado que la relación velocidad-vorticidad es lineal)

$$
\dot{\mathbf{x}}=\sum_{\alpha=1}^{N} \nabla^{\perp} \psi_{\alpha}(\mathbf{x}, t) .
$$


Entonces, dado que cada vórtice puntual se mueve con la velocidad local de el fluido y la vorticidad es una cantidad conservada, tenemos las ecuaciones para la colección de $N$-vórtices

$$
\dot{\mathbf{x}_{\beta}}=\sum_{\alpha \neq \beta}^{N} \nabla^{\perp} \psi_{\alpha}\left(\mathbf{x}_{\beta}, t\right)
$$

donde $\beta=1, \ldots, N$.

Interacción de $N$ vórtices puntuales. Desarrollando (3.20) podemos ahora escribir las ecuaciones de movimiento para $N$ vórtices en el plano:

$$
\begin{aligned}
& \dot{x}_{\alpha}=-\frac{1}{2 \pi} \sum_{\beta \neq \alpha}^{N} \frac{\Gamma_{\beta}\left(y_{\alpha}-y_{\beta}\right)}{l_{\beta \alpha}^{2}} \\
& \dot{y}_{\alpha}=\frac{1}{2 \pi} \sum_{\beta \neq \alpha}^{N} \frac{\Gamma_{\beta}\left(x_{\alpha}-x_{\beta}\right)}{l_{\beta \alpha}^{2}}
\end{aligned}
$$

donde $l_{\beta \alpha}=\left\|\mathbf{x}_{\beta}-\mathbf{x}_{\alpha}\right\|$ es la distancia entre los vórtices. El sistema puede ser expresado más compactamente en la notación compleja $z_{\alpha}(t)=x_{\alpha}(t)+i y_{\alpha}(t)$, como sigue

$$
\dot{z}_{\alpha}=\frac{i}{2 \pi} \sum_{\beta \neq \alpha}^{N} \Gamma_{\beta} \frac{z_{\alpha}-z_{\beta}}{\left|z_{\alpha}-z_{\beta}\right|^{2}}
$$

o equivalentemente

$$
\dot{z}_{\alpha}^{*}=\frac{1}{2 \pi i} \sum_{\beta \neq \alpha}^{N} \frac{\Gamma_{\beta}}{z_{\alpha}-z_{\beta}}
$$

asumiendo que todos los vórtices $\Gamma_{\alpha}$ son reales y $\alpha=1, \ldots, N$.

En el capítulo 4 elaboraremos sobre estas ecuaciones.

\subsection{Otras dinámicas de vorticidad en el plano}

Existen flujos que, hasta cierto punto, comparten propiedades cualitativas con el flujo debido a un vórtice puntual. Veamos un par de ejemplos. 


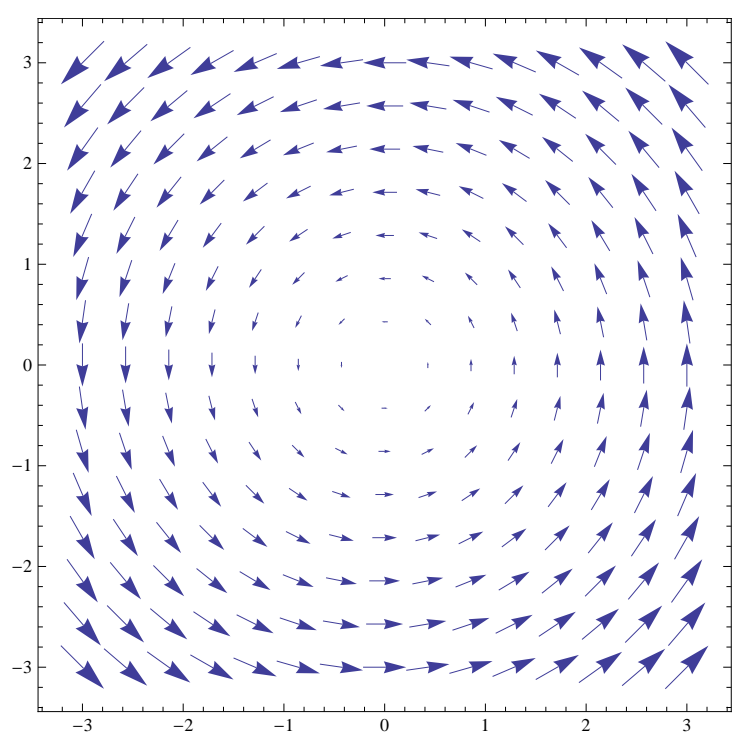

Figura 3.4: Campo de velocidad para el flujo de cuerpo sólido.

\section{Rotación de un cuerpo sólido}

$$
\begin{aligned}
u_{\theta}(r, \theta, z) & =\Omega r \\
u_{r} & =0 \\
u_{z} & =0 \\
\omega_{z} & =\frac{1}{r}\left(\frac{\partial\left(r u_{\theta}\right)}{\partial r}-\frac{\partial u_{r}}{\partial \theta}\right) \\
& =2 \Omega
\end{aligned}
$$

En la figura 3.4 se ilustra el flujo de cuerpo sólido.

\section{Vórtice de Rankine}

Este flujo corresponde a la rotación de un cuerpo sólido dentro de un 


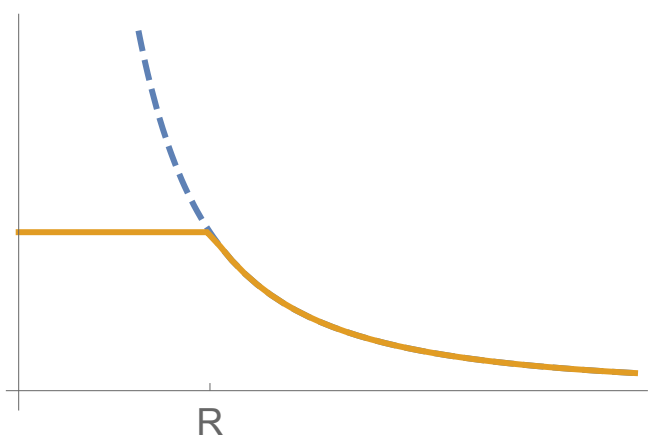

(a)

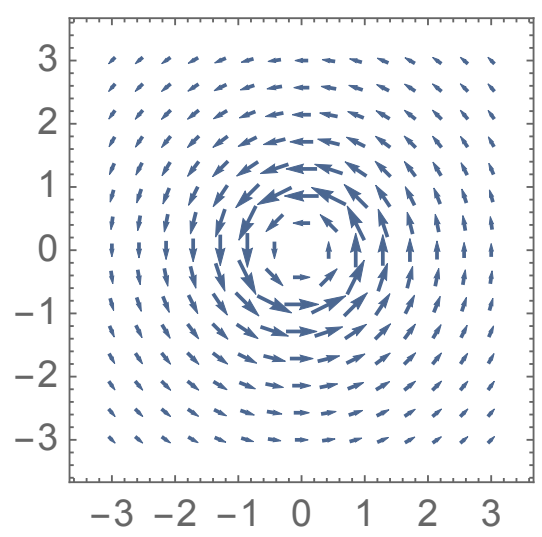

(b)

Figura 3.5: (a) Magnitud de la velocidad como función del radio para el flujo de Rankine (línea continua) y el flujo de un vórtice puntual (línea discontinua). (b) Campo de velocidad para el flujo de Rankine.

círculo de radio $R$ y un vórtice puntual fuera del círculo.

$$
\begin{aligned}
u_{\theta}(r, \theta, z) & = \begin{cases}\left(\frac{\Gamma}{2 \pi R^{2}}\right) r, & r \leq R \\
\frac{\Gamma}{2 \pi r}, & r>R\end{cases} \\
\omega_{z} & = \begin{cases}\frac{\Gamma}{\pi R^{2}}, & r \leq R \\
\delta(r), & r>R\end{cases} \\
u_{r} & =0 \\
u_{z} & =0
\end{aligned}
$$

Podemos imaginar a una hélice de motor en un líquido, al girar la hélice obliga al fluido a moverse, lo cual esta originando el comportamiento de la rotación del cuerpo sólido, mientras que el fluido fuera de la hélice es más lento el movimiento y se comporta como un vórtice puntual.

En la figura 3.5 se ilustra el modelo del flujo de Rankine.

\subsection{Integración del problema de tres vórtices}

En esta sección discutimos, en su contexto histórico, la integrabilidad del problema de tres vórtices y comentamos brevemente sobre la aportación de esta tesis al tema de integrabilidad. 
Consideremos $z_{1}(t), \cdots, z_{N}(t)$ las posiciones de $N$ vórtices y $\Gamma_{1}, \cdots, \Gamma_{N}$ sus respectivas vorticidades, hemos visto que la dinámica de vorticidad del sistema es expresado como

$$
\dot{z}_{\alpha}=\frac{i}{2 \pi} \sum_{\alpha \neq \beta}^{N} \Gamma_{\beta} \frac{z_{\alpha}-z_{\beta}}{\left|z_{\alpha}-z_{\beta}\right|^{2}} .
$$

Asociado a la ecuación anterior, tenemos 2 cantidades, una la denotamos como $I$ momento de inercia y $\mathcal{H}$ la energía. Ambas cantidades son conservadas, lo cual nos ayuda a reducir nuestro problema, es decir, reducimos la dimensión de nuestro sistema. Ahora, si nuestro centro de vorticidad está ubicado (o anclado) en el origen $z=0$, reducimos nuestro sistema en dos dimensiones más. Como nuestras ecuaciones del sistema son invariantes bajo traslaciones y rotaciones, podemos en principio olvidar información de manera inteligente, ya que podremos escribir nuestro sistema en términos de las distancias de los tres lados del triángulo que forma la configuración. Entonces tendremos un paso más que logra reducir finalmente el problema de $\mathbb{R}^{6}$ a $\mathbb{R}$. Podemos decir entonces que el sistema es integrable.

Los hechos anteriores, sus ideas y cálculos, se deben a los trabajos de Poincaré, Gröbli, Synge y Aref. Para mayor referencia se puede consultar [7].

Poincaré fue uno de los primeros en darse cuenta de la integrabilidad del sistema, la cual se pudo explicar mediante consideraciones sobre las simetrías e integrales primeras.

El primero en introducir el modelo de las ecuaciones que rigen el movimiento de tres vórtices fue Helmholtz (1858), quien dedujo esencialmente las ecuaciones 4.1), aunque sin ocupar la notación compleja que nosotros ocupamos.

Kirchhoff, para el sistema descrito por Helmholtz, hace la observación de que el sistema se puede escribir en forma hamiltoniana. Mediante algunos cálculos obtiene que el centroide (centro de vorticidad), el hamiltoniano $\mathcal{H}$ y el momento de Inercia $I$ son constantes. El problema de 2 vórtices lo tenía completamente entendido.

Tanto Kirchhoff como Poincaré se habían percatado que utilizando resultados generales de sistemas hamiltonianos el problema de tres vórtices tenía que ser integrable. Poincaré consideró el problema de tres vórtices en su artículo [32] Théorie des Tourbillons en 1893.

El primero en describir en detalle la integrabilidad del problema de tres vórtices es Gröbli. Describe el sistema con la ecuaciones de movimiento, pero 
en términos de los cuadrados de las distancias entre los vórtices y ocupando el hecho de que $\mathcal{H}=$ const. e $I=$ const., con esto logra describir la forma del triángulo, independientemente de su orientación en el plano, a lo cual nosotros llamamos reducción del sistema. En esencia, Gröbli llega a nuestras ecuaciones (5.1) para tres vórtices.

Un aspecto que se aborda en la presente tesis, que quizá no conocía Gröbli, es el proceso de reducción hamiltoniana. Esto es, notamos que las ecuaciones (5.1) no son hamiltonianas, pero sin embargo es posible encontrar un sistema de coordenadas, con el cual las ecuaciones de movimiento sí tienen una estructura hamiltoniana. Podemos decir que uno de los objetivos principales de esta tesis es discutir la estructura geométrica y de Poisson que dan lugar a dicha reducción hamiltoniana. 


\section{Capítulo 4}

\section{El problema de $N$ vórtices en el plano}

En este capítulo estableceremos la estructura hamiltoniana de las ecuaciones de movimiento del problema de $N$ vórtices en el plano, estableciendo algunas propiedades básicas y cantidades conservadas. Adicionalmente, contemplaremos algunos casos particulares con el objeto de ganar intuición sobre la dinámica de vórtices puntuales.

\subsection{Formulación general}

Las ecuaciones para una colección de vórtices, cada una con vorticidad $\Gamma_{\alpha} \in \mathbb{R}$, localizado en $z_{\alpha}=x_{\alpha}+i y_{\alpha}$ esta dada por

$$
\dot{z}_{\alpha}=\frac{i}{2 \pi} \sum_{\alpha \neq \beta}^{N} \Gamma_{\beta} \frac{z_{\alpha}-z_{\beta}}{\left|z_{\alpha}-z_{\beta}\right|^{2}}
$$

El sistema tiene la forma Hamiltoniana siguiente

$$
\Gamma_{\alpha} \dot{x}=\frac{\partial \mathcal{H}}{\partial y_{\alpha}}, \quad \Gamma_{\alpha} \dot{y}=-\frac{\partial \mathcal{H}}{\partial x_{\alpha}}
$$

$\alpha=1, \ldots, N$, con

$$
\mathcal{H}=-\frac{1}{4 \pi} \sum_{\beta \neq \alpha}^{N} \Gamma_{\alpha} \Gamma_{\beta} \log \left(l_{\alpha \beta}\right)
$$


donde $l_{\alpha \beta}=\left|z_{\alpha}-z_{\beta}\right|$. Por conveniencia usaremos la notación $\Gamma=\sum_{\alpha}^{N} \Gamma_{\alpha}$ que denota la vorticidad total en el plano. El sistema (4.1) tiene las siguientes cuatro cantidades conservadas $(\mathcal{H}, Q, P, I)$, donde $\mathcal{H}$, la energía de interacción, queda definida por la ecuación 4.2. Ahora si tomamos

$$
\begin{aligned}
\frac{d \mathcal{H}}{d t} & =\frac{\partial \mathcal{H}}{\partial x} \dot{x}+\frac{\partial \mathcal{H}}{\partial y} \dot{y} \\
& =0
\end{aligned}
$$

observamos que $\mathcal{H}$ es una cantidad conservada.

La siguiente cantidad conservada es el momento de vorticidad, denotado por

$$
Q+i P=\sum_{\alpha=1}^{N} \Gamma_{\alpha} z_{\alpha}
$$

Tomando

$$
\begin{aligned}
\frac{d(Q+i P)}{d t} & =\frac{d}{d t} \sum_{\alpha=1}^{N} \Gamma_{\alpha} z_{\alpha} \\
& =\sum_{\alpha=1}^{N} \Gamma_{\alpha} \dot{z}_{\alpha} \\
& =\sum_{\alpha, \beta}^{N} D_{\alpha \beta} \\
& =0
\end{aligned}
$$

donde $D_{\alpha \beta}=\frac{i}{2 \pi} \Gamma_{\alpha} \Gamma_{\beta} \frac{z_{\alpha}-z_{\beta}}{\left|z_{\alpha}-z_{\beta}\right|^{2}}$ y $D_{\alpha \beta}=-D_{\alpha \beta}$. Entonces, $Q+i P$ es una cantidad conservada.

Si $\Gamma=\sum_{k} \Gamma_{k} \neq 0$ definimos el centro de vorticidad como

$$
Z_{0}=\frac{Q+i P}{\Gamma}
$$

La última cantidad conservada es el momento angular (también llamada impulso angular ó segundo momento de circulación) del sistema, denotado por

$$
I=\sum_{\alpha=1}^{N} \Gamma_{\alpha}\left|z_{\alpha}\right|^{2}
$$


En efecto, observamos que

$$
\begin{aligned}
\frac{d I}{d t} & =\frac{d}{d t} \sum_{\alpha=1}^{N} \Gamma_{\alpha}\left|z_{\alpha}\right|^{2} \\
& =\sum_{\alpha=1}^{N} \Gamma_{\alpha} \frac{d}{d t}(z \cdot \bar{z}) \\
& =\sum_{\alpha, \beta=1}^{N} \Gamma_{\alpha}(\dot{z} \bar{z}+z \dot{\bar{z}}) \\
& =0 .
\end{aligned}
$$

por tanto, es una cantidad conservada. (Notemos que este resultado se puede ver como un caso particular de la conservación de la integral definida en en (3.17).)

Es fácil demostrar que si colocamos el centro de vorticidad en el origen entonces una expresión equivalente para el momento angular es

$$
I=\frac{1}{\Gamma} \sum_{j<k} \Gamma_{j} \Gamma_{k}\left|z_{j}-z_{k}\right|^{2},
$$

donde $\Gamma$ es la vorticidad total. Esta expresión será importante para nosotros más adelante.

Debido a que el braquet canónico de Poisson entre dos funciones $f(\mathbf{z}), g(\mathbf{z})$ para la variable de configuración $\mathbf{z}=\left(z_{1}, \ldots, z_{N}\right)$ es

$$
\{f, g\}=\sum_{\alpha=1}^{N} \frac{1}{\Gamma_{\alpha}}\left(\frac{\partial f}{\partial x_{\alpha}} \frac{\partial g}{\partial y_{\alpha}}-\frac{\partial f}{\partial y_{\alpha}} \frac{\partial g}{\partial x_{\alpha}}\right) .
$$

entonces, tenemos las siguientes tres cantidades que están en involución:

1. $\{\mathcal{H}, I\}=0$

2. $\left\{\mathcal{H}, P^{2}+Q^{2}\right\}=0$

3. $\left\{P^{2}+Q^{2}, I\right\}=0$

en adición a estas, tenemos

1. $\{Q, P\}=\Gamma$ 
2. $\{Q, I\}=2 P$

3. $\{P, I\}=-2 Q$

dando lugar al siguiente resultado fundamental

Teorema 4.1.1. El problema de $N$-vórtices para $N \leq 3$ es integrable para todos los valores de $\Gamma_{\alpha}$. Si $\Gamma=0$, el problema de 4-vórtices es integrable.

Demostración. Para $N=3$, sabemos que las tres cantidades $\mathcal{H}, I, P^{2}+Q^{2}$ son constantes, están en involución y son funcionalmente independientes, como un sistema Hamiltoniano de dimensión $2 N$ es integrable si tiene $N$ funciones en involución, obtenemos la integración del sistema. Si $\Gamma=0$ entonces las cuatro cantidades $\mathcal{H}, I, P, Q$ son constantes y están en involución, de igual forma obtenemos lo deseado.

Terminemos esta subsección apuntando que la forma simpléctica asociada al braquet de Poisson (4.5) está dado por

$$
\Omega(\mathbf{z}, \mathbf{w})=-\operatorname{Im} \sum_{\alpha=1}^{N} \Gamma_{\alpha} z_{\alpha} \bar{w}_{\alpha} .
$$

\subsubsection{Teorema de Synge}

Teorema 4.1.2 (Synge). 1. Dado una configuración de vórtices, si las vorticidades de todos los vórtices son revertidas, el sistema se podrá reconstruir mediante la sucesión de configuraciones a través de la cual vino.

2. Si una configuración es colineal al tiempo $t=t_{0}$, entonces la configuración en los tiempos $t=t_{0} \pm \tau$ son reflexiones uno del otro para todos los valores de $\tau$.

3. Un sistema no puede pasar a través de más de dos distintas configuraciones colineales. Los tiempos requeridos para pasar de una configuración colineal a otra son todos los mismos.

4. Supongamos que existen dos sistemas $S_{1}$ y $S_{2}$ de vórtices, tal que cada uno consiste de el mismo número de vórtices, con la vorticidad de cada vórtice en $S_{2}$ siendo $\lambda^{2}$ veces la fuerza de cada en $S_{1}$. Supongamos 
además que las configuraciones inicialmente son similares, sin reflexión, con las longitudes en $S_{2}$ siendo $L$ veces estas en $S_{1}$, donde $L$ es una constante. Entonces la subsucesión de configuración de $S_{2}$ después del tiempo $t_{2}$ es similar, sin reflexión, a la configuración de $S_{1}$ después del tiempo $t_{1}$, donde $t_{2}=t_{1}\left(\frac{L^{2}}{\lambda^{2}}\right)$

5. Si las vorticidades de todos los vórtices tienen el mismo signo, sus distancias están acotadas por arriba y por abajo, para todos los tiempos.

\subsection{Casos especiales}

Consideremos a continuación algunos casos especiales en el problema de $N$-vórtices.

\subsection{1. $\quad$ El problema restringido de $N$-vórtices}

Definición 4.2.1. Cuando una de las vorticidades del sistema de $N$-vórtices es tomada como cero, i.e. $\Gamma_{\alpha}=0$ para algún $\alpha, 1 \leq \alpha \leq N$, uno se refiere a esto como un problema de $N$-vórtices restringido.

Esta terminología es prestada de la literatura de mecánica celeste, donde uno se refiere a un problema de $N$-cuerpos restringido cuando una de las masas es tomada como cero.

Sin pérdida de la generalidad tomamos $\Gamma_{N}=0$. Las ecuaciones de movimiento para una partícula localizada en la posición $z=z_{N}$ se desacoplan de las ecuaciones que gobiernan los $N-1$ vórtices restantes,

$$
\begin{aligned}
\dot{\bar{z}} & =-\frac{i}{2 \pi} \sum_{\beta=1}^{N-1} \frac{\Gamma_{\beta}}{z-z_{\beta}} \\
\dot{\bar{z}}_{\alpha} & =-\frac{i}{2 \pi} \sum_{\beta \neq \alpha} \frac{\Gamma_{\beta}}{z_{\alpha}-z_{\beta}}
\end{aligned}
$$

para $\alpha=1, \ldots, N-1$.

En la sección 5.1 continuaremos la discusión de estas ecuaciones para el caso $N=3$. 


\subsubsection{Soluciones autosimilares}

En esta parte definiremos una clase especial de movimiento de vórtices, llamados evolución-autosimilar.

Consideremos la solución $\zeta_{\alpha}(t), \alpha=1, \ldots, N$, a un sistema de $N$ vórtices puntuales. Tenemos la siguiente

Definición 4.2.2. Un sistema de vórtices evoluciona auto-similarmente si $\zeta_{\alpha}(t)=\vartheta+z_{\alpha}(t)$ con $z_{\alpha}=\lambda_{\alpha} f(t)$ para cada $\alpha=1, \ldots, N$, donde $\vartheta$ y las $\lambda_{\alpha} \in \mathbb{C}$ son factores escalares complejo-valuadas y $f(t) \equiv r(t) \exp (i \theta(t))$ es una función complejo-valuada del tiempo.

A la constante $\vartheta$ la llamaremos origen de la solución autosimilar.

Comenzamos el estudio de las soluciones autosimilares planteándonos las siguientes preguntas:

1. Determinar cómo se define $\vartheta$ en términos de las vorticidades.

2. ¿Está definido $\vartheta$ para cualquier combinación de vorticidades?

3. ¿Cómo se interpreta geométricamente la definición de soluciones autosimilares?

4. ¿Qué propiedades debe tener una configuración autosimilar?

Sabemos que

$$
Q+i P=\sum \Gamma_{\alpha} \zeta_{\alpha}(t)=\vartheta \sum \Gamma_{\alpha}+\sum \Gamma_{\alpha} z_{\alpha}(t)
$$

es una cantidad conservada. Por lo tanto

$$
0=\frac{d}{d t}(Q+i P)=\frac{d}{d t}\left[\vartheta \sum \Gamma_{\alpha}+f(t) \sum \Gamma_{\alpha} \lambda_{\alpha}\right]=\dot{f} \sum \Gamma_{\alpha} \lambda_{\alpha}
$$

Consideremos los siguientes casos:

Caso 1: $\dot{f}$ no es idénticamente cero. Entonces $\sum \Gamma_{\alpha} \lambda_{\alpha}=0$ y por lo tanto

$$
\begin{aligned}
Q+i P & =\vartheta \sum \Gamma_{\alpha}+f(t) \sum \Gamma_{\alpha} \lambda_{\alpha} \\
& =\vartheta \sum \Gamma_{\alpha} .
\end{aligned}
$$


Sustituyendo en la ecuación (4.6) obtenemos que

$$
\sum \Gamma_{\alpha} z_{\alpha}(t)=0
$$

Geométricamente podemos interpretar esta ecuación diciendo que, en cualquier instante $t$, los vectores $\mathbf{v}_{\alpha}:=\Gamma_{\alpha} z_{\alpha}$ forman los lados de un $N$-gono.

Adicionalmente, si $\sum \Gamma_{\alpha} \neq 0$ entonces

$$
\vartheta=\frac{Q+i P}{\sum \Gamma_{\alpha}}=: Z_{0}, \quad \text { el centro de vorticidad. }
$$

Por otro lado, si $\sum \Gamma_{\alpha}=0$ entonces $\vartheta$ puede tomar cualquier valor constante arbitrario y $Q+i P=0$.

Caso 2: $\quad \dot{f} \equiv 0$. En este caso la solución autosimilar es de hecho un equilibrio. Si $\sum \Gamma_{\alpha} \neq 0$ entonces definiendo $\vartheta$ como el centro de vorticidad tenemos nuevamente que $\sum \Gamma_{\alpha} z_{\alpha}=0$ y se sigue la misma interpretación geométrica que obtuvimos en el caso 1 . Si la vorticidad total $\sum \Gamma_{\alpha}$ es cero entonces $\sum \Gamma_{\alpha} z_{\alpha}$ es independiente de la elección de $\vartheta$ y ya no necesariamente tenemos la interpretación geométrica que obtuvimos en el caso 1. (No es claro que existan soluciones de equilibrio para el caso $\sum \Gamma_{\alpha}=0$; por ejemplo, es fácil checar, usando la ecuación (4.8), que no existen equilibrios para tres vórtices con $\Gamma_{1}=2, \Gamma_{2}=-1$ y $\Gamma_{3}=-1$.)

Obtengamos a continuación cómo debe ser $f(t)$ para que la solución sea autosimilar. Sin perdida de generalidad podemos considerar $r(0)=1$, dado que $\operatorname{los} \lambda_{\alpha}$ pueden tener módulos arbitrarios. Consideremos el sistema de vorticidad del plano escrito como

$$
\dot{z}_{\alpha}=\frac{i}{2 \pi} \sum_{\beta \neq \alpha}^{N} \frac{\Gamma_{\beta}}{\left(\bar{z}_{\alpha}-\bar{z}_{\beta}\right)}
$$

para $\alpha=1 \ldots, N$. Asumiendo que el sistema evoluciona auto-similarmente, entonces tenemos

$$
\lambda_{\alpha} \dot{f} \bar{f}=\frac{i}{2 \pi} \sum_{\beta \neq \alpha}^{N} \frac{\Gamma_{\beta}}{\left(\bar{\lambda}_{\alpha}-\bar{\lambda}_{\beta}\right)}
$$

que da la ecuación dinámica para $f(t)$, de donde tenemos el siguiente sistema

$$
\dot{f} \bar{f}=C \equiv A+i B
$$


junto con el sistema algebraico de $N$ ecuaciones complejas

$$
\lambda_{\alpha} C=\frac{i}{2 \pi} \sum_{\beta \neq \alpha}^{N} \frac{\Gamma_{\beta}}{\left(\bar{\lambda}_{\alpha}-\bar{\lambda}_{\beta}\right)}
$$

La ecuación 4.8 da las condiciones para que una configuración inicial de vórtices evolucione autosimilarmente. Notemos entonces que la condición necesaria $\sum \Gamma_{\alpha} \lambda_{\alpha}=0$ (que hemos interpretado geométricamente en términos de los lados de un $N$-gono) no es suficiente, pues se requiere la condición 4.8 que es más fuerte.

Para resolver el sistema (4.7) sea $f(t)=r(t) \exp (i \theta(t))$, que da el sistema acoplado amplitud-fase

$$
\begin{array}{rlr}
\frac{1}{2} \frac{d\left(r^{2}\right)}{d t} & =A, \quad r(0)=1 \\
\frac{d \theta}{d t} & =\frac{B}{r^{2}}
\end{array}
$$

cuya solución es

$$
\begin{aligned}
& r(t)=\sqrt{2 A t+1} \\
& \theta(t)= \begin{cases}\frac{B}{2 A} \log (2 A t+1) & (A \neq 0) \\
B t+\theta(0) & (A=0)\end{cases}
\end{aligned}
$$

Los vórtices chocan autosimilarmente (en su centro de vorticidad) si $r\left(t^{*}\right)=0$ para algún $t^{*}>0$. De aquí, si $A<0$, entonces $t^{*}=-\frac{1}{2 A}>0$. Resolviendo algebraicamente el sistema (4.8) para las constantes $\lambda_{\alpha}$ para $N$ general es complicado, pero un tratamiento sistemático puede ser dado. Más aún, no es difícil construir casos especiales, como el ejemplo 4.2.4.

Observación 4.2.3. Etimológicamente, una similaridad es una transformación que re-escala las distancias entre cualesquiera dos puntos usando siempre el mismo factor de escala. Así, una solución autosimilar en el sentido de la definición 4.2.2 es una similaridad; pero no viceversa. Por ejemplo, consideremos el sistema de dos vórtices con $\Gamma_{1}=1, \Gamma_{2}=-1$ y posiciones iniciales en $z_{1}(0)=i$ y $z_{2}(0)=-i$. De las ecuaciones de movimiento (3.23) se obtiene fácilmente que, denotando $\zeta=z_{2}-z_{1}, \dot{\zeta}(t)=0$ de donde $\zeta(t)=-2 i$ y se sigue que

$$
z_{1}(t)=i+\frac{t}{4 \pi}, \quad z_{2}(t)=-i+\frac{t}{4 \pi} .
$$


Vemos entonces que esta solución no es autosimilar en el sentido de la definición 4.2.2 pero sí es una similaridad, pues es una translación horizontal. (Expandiendo nuestra definición permitiendo que ७ sea variable abarcaríamos este ejemplo, en este caso con $\vartheta=t / 4 \pi$. Sin embargo, siguiendo a [30], preferimos mantener la definición restringida.)

Ejemplo 4.2.4 (Kimura(1988)). Tomando como caso especial $\left(\Gamma_{1}, \Gamma_{2}, \Gamma_{3}\right)=$ $(2,2,-1)$ con condiciones iniciales, tomando $z_{i}(0)=x_{1}(0)+i y(0)$ con $i=1,2,3$, tenemos

$$
\begin{aligned}
& z_{1}(0)=\left(\frac{3+\sqrt{3} \cos \theta}{6}+i \frac{\sqrt{3} \operatorname{sen} \theta}{6}\right) l_{12} \\
& z_{2}(0)=\left(\frac{-3+\sqrt{3} \cos \theta}{6}+i \frac{\sqrt{3} \operatorname{sen} \theta}{6}\right) l_{12} \\
& z_{3}(0)=\left(\frac{2 \sqrt{3} \cos \theta}{3}+i \frac{2 \sqrt{3} \operatorname{sen} \theta}{3}\right) l_{12}
\end{aligned}
$$

donde $l=\left\|x_{1}-x_{2}\right\|$ y $\operatorname{los} \theta$ son arbitrarios. El centro de vorticidad de esta configuración esta en el origen donde chocan los vórtices. Resolviendo para $A$ y $B$ tenemos

$$
\begin{aligned}
& A=-\frac{6 \operatorname{sen} 2 \theta}{(5-3 \cos 2 \theta) l_{12}^{2}} \\
& B=\frac{18-6 \cos 2 \theta}{(5-3 \cos 2 \theta) l_{12}^{2}}
\end{aligned}
$$

con un tiempo de colisión $t^{*}$ dado por

$$
t^{*}=\frac{5-3 \cos 2 \theta}{12 \operatorname{sen} 2 \theta} l_{12}^{2} .
$$

Los vórtices se mueven en espiral, colisionando en un tiempo finito si $\theta \neq$ $\pi / 4(\bmod \pi / 2)$. 


\section{Capítulo 5}

\section{El problema de tres vórtices en el plano}

En este capítulo describiremos el movimiento de tres vórtices en el plano, partiendo de las ecuaciones que gobiernan el movimiento de dos vórtices, de ahí pasaremos a ver el movimiento de tres, caracterizaremos todos sus estados fijos y de equilibrio relativos y finalmente podremos escribir las ecuaciones de movimiento en términos de sus distancias al cuadrado y a lo que nos lleva tal descripción.

\subsection{El problema restringido de tres vórtices}

En esta sección comenzaremos con un ejemplo, para contrastar con la solución del problema restringido de 3-cuerpos de mecánica celeste.

Ejemplo 5.1.1 (Koiller, Carvalho (1985)). Las ecuaciones que gobiernan el movimiento de dos vórtices son

$$
\begin{aligned}
& \dot{z}_{1}=\frac{i \Gamma_{2}}{2 \pi} \cdot \frac{z_{1}-z_{2}}{\left|z_{1}-z_{2}\right|^{2}} \\
& \dot{z}_{2}=\frac{i \Gamma_{1}}{2 \pi} \cdot \frac{z_{2}-z_{1}}{\left|z_{1}-z_{2}\right|^{2}}
\end{aligned}
$$

Consideraremos el caso $\Gamma_{1}+\Gamma_{2} \neq 0$, entonces los 2 movimientos primarios están en órbitas circulares alrededor de el centro de vorticidad

$$
C_{0}=\frac{\Gamma_{1} z_{1}+\Gamma_{2} z_{2}}{\left(\Gamma_{1}+\Gamma_{2}\right)}
$$


con frecuencia

$$
\omega=\frac{\Gamma_{1}+\Gamma_{2}}{2 \pi D^{2}}
$$

y $D$ es la distancia entre 2 vórtices. Consideramos el movimiento de un tercer vórtice, de vorticidad $\Gamma_{3} \approx 0$. Su ecuación que gobierna el movimiento, asumiendo que no afecta el movimiento de $\Gamma_{1}$ y $\Gamma_{2}$, es

$$
\dot{z}_{3}=\frac{i \Gamma_{1}}{2 \pi} \cdot \frac{\left(z_{3}-z_{1}\right)}{\left|z_{3}-z_{1}\right|^{2}}+\frac{i \Gamma_{2}}{2 \pi} \cdot \frac{z_{3}-z_{2}}{\left|z_{3}-z_{2}\right|^{2}}
$$

donde se han fijado las coordenadas de manera que el centro de vorticidad este en el origen. Ahora transformamos a un marco de referencia rotante en el cual los vórtices $\left(\Gamma_{1}, \Gamma_{2}\right)$ están en reposo,

$$
\dot{\xi}_{j}==\exp (-i \omega t) z_{j}, \quad j=1,2,3
$$

dando una ecuación con coeficientes periódicos

$$
\dot{\xi}_{3}=-i \omega \xi_{3}+\frac{i \Gamma_{1}}{2 \pi} \cdot \frac{\xi_{3}-\xi_{1}}{\left|\xi_{3}-\xi_{1}\right|^{2}}+\frac{i \Gamma_{2}}{2 \pi} \cdot \frac{\xi_{3}-\xi_{2}}{\left|\xi_{3}-\xi_{2}\right|^{2}}
$$

con $\Gamma_{1} \xi_{1}+\Gamma_{2} \xi_{2}=0$. Como en el problema restringido de tres cuerpos, es conveniente normalizar el problema escogiendo unidades de longitud y tiempo tales que

$$
\begin{aligned}
\Gamma_{1}+\Gamma_{2} & =2 \pi \\
\left|\xi_{1}-\xi_{2}\right| & =1
\end{aligned}
$$

y escogemos las coordenadas $\xi_{1}, \xi_{2}$ tales que coincidan con el eje real. El sistema puede ser convenientemente expresado por

$$
\begin{aligned}
\xi_{1} & =-\lambda \\
\xi_{2} & =1-\lambda \\
\Gamma_{2} & =2 \pi \lambda
\end{aligned}
$$

para $\lambda \in(-\infty, \infty)$. La ecuación para $\xi_{3} \equiv u+i v$ es un sistema Hamiltoniano, con el Hamiltoniano dado por

$$
\begin{aligned}
\mathcal{H}(u, v)=-\frac{1}{2}\left(u^{2}+v^{2}\right)+(1-\lambda) \log \left(\sqrt{(u+\lambda)^{2}+v^{2}}\right. & \\
& +\lambda \log \left(\sqrt{(u+\lambda-1)^{2}+v^{2}}\right) .
\end{aligned}
$$




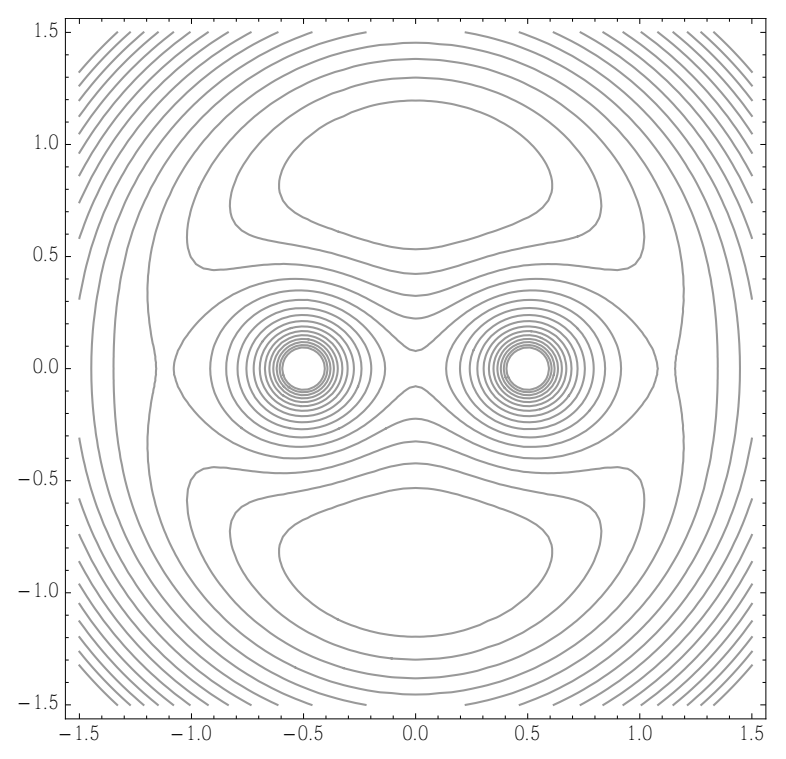

Figura 5.1: Retrato fase para el problema restringido de tres vórtices.

Las curvas de nivel se muestran en la figura 5.1, para el caso simétrico de valores primarios iguales, es decir $\lambda=\frac{1}{2}$. Tenemos dos equilibrios correspondientes a dos configuraciones de triángulo equilátero en los puntos fijos $\xi= \pm i \sqrt{\frac{3}{2}}$, y tres estados colineales en $\xi=0, \pm \frac{\sqrt{5}}{2}$. Para el caso general $0<\lambda<1$ las mismas cinco posibilidades de equilibrio están presentes. Estas coordenadas, por supuesto, dependen de un valor específico de $\lambda$. Estos son los análogos a los puntos de Lagrange para el problema restringido de tres-cuerpos. La estabilidad del equilibrio puede ser determinada mediante la evaluación de la matriz Hessiana en el punto crítico, dando

$$
\begin{array}{rlrl}
\mathcal{H}^{\prime \prime}(0,0) & =\left(\begin{array}{cc}
-5 & 0 \\
0 & 3
\end{array}\right) & & \text { (punto silla) } \\
\mathcal{H}^{\prime \prime}\left( \pm \frac{\sqrt{5}}{2}, 0\right) & =\left(\begin{array}{cc}
-\frac{5}{2} & 0 \\
0 & \frac{1}{2}
\end{array}\right) & \text { punto silla } \\
\mathcal{H}^{\prime \prime}\left(0 \pm \frac{\sqrt{3}}{2}\right) & =\left(\begin{array}{cc}
-\frac{1}{2} & 0 \\
0 & -\frac{3}{2}
\end{array}\right) & \text { (equilibrio estable) }
\end{array}
$$

dando que las configuraciones de el triángulo equilátero son no- linealmente estables, mientras que los estados colineales son inestables. 
5.2 Ecuaciones de movimiento usando el cuadrado de las distancias

\subsection{Ecuaciones de movimiento usando el cua- drado de las distancias}

Para el problema general de tres vórtices, las ecuaciones de movimiento se pueden escribir en términos de las distancias al cuadrado de los mismos. Ese

es el punto de vista que adoptamos en esta sección, tomando como motivación las ideas planteadas en [16].

Comenzamos probando el siguiente resultado:

Teorema 5.2.1. Para un sistema de vórtices puntuales $\left\{\Gamma_{1}, \ldots, \Gamma_{N}\right\}$ las ecuaciones de movimiento estás dadas por

$$
\dot{b}_{i j}=\frac{2}{\pi} \sum_{k \neq i, j} \Gamma_{k} A_{i j k}\left(\frac{1}{b_{i k}}-\frac{1}{b_{k j}}\right), \quad i<j
$$

donde $b_{i j}=\left|z_{i}-z_{j}\right|^{2}, z_{i} \in \mathbb{C}$ denota la posición del $i$-ésimo vórtice y $A_{i k j}$ es el área orientada del triángulo $\triangle\left(z_{i}, z_{k}, z_{j}\right)$.

El área $A_{i j k}$ queda determinada en términos de $b_{i j}, b_{j k}$ y $b_{k i}$ usando la fórmula de Herón; ver la ecuación (5.5) más adelante. La ambigüedad del signo de la raíz cuadrada en la fórmula de Herón implica que, de hecho, las ecuaciones (5.1) definen dos campos vectoriales en coordenadas dadas por los cuadrados de las distancias.

Para probar el teorema, primero observamos lo siguiente

$$
\operatorname{Im}\left(\frac{z_{1}}{z_{2}}+\frac{z_{2}}{z_{1}}\right) \equiv \operatorname{Im}\left(\bar{z}_{1} z_{2}\right)\left(\frac{1}{\left|z_{1}\right|^{2}}-\frac{1}{\left|z_{2}\right|^{2}}\right)
$$

para todo $z_{1}, z_{2} \in \mathbb{C} \backslash\{0\}$; esta identidad se demuestra fácilmente.

Demostración del teorema 5.2.1. Sea $\omega_{i j}=z_{i}-z_{j}$ para $i \neq j$. Entonces

$$
\dot{w}_{i j}=\frac{d}{d t}\left(w_{i j} \bar{w}_{i j}\right)=\dot{w}_{i j} \bar{w}_{i j}+w_{i j} \dot{\bar{w}}_{i j}
$$

escribiendo nuevamente las ecuaciones tenemos lo siguiente

$$
\dot{w}_{i j}=\frac{i}{2 \pi}\left[\sum_{k \neq i, j} \frac{\Gamma_{k}}{\bar{w}_{i k}}+\frac{\Gamma_{j}}{\bar{w}_{i j}}-\sum_{k \neq i, j} \frac{\Gamma_{k}}{\bar{w}_{j k}}-\frac{\Gamma_{i}}{\bar{w}_{j i}}\right]
$$


por lo tanto tenemos, por una parte

$$
\dot{w}_{i j} \bar{w}_{i j}=\frac{i}{2 \pi}\left[\sum_{k \neq i, j} \Gamma_{k}\left(\frac{\bar{w}_{i j}}{\bar{w}_{i k}}+\frac{\bar{w}_{i j}}{\bar{w}_{k j}}\right)+\Gamma_{i}+\Gamma_{j}\right]
$$

$\mathrm{y}$

$$
w_{i j} \dot{\bar{w}}_{i j}=-\frac{i}{2 \pi}\left[\sum_{k \neq i, j} \Gamma_{k}\left(\frac{w_{i j}}{w_{i k}}+\frac{w_{i j}}{w_{k j}}\right)+\Gamma_{i}+\Gamma_{j}\right] .
$$

Sumando ambas ecuaciones y tomando en cuenta que $w_{i j}=w_{i k}+w_{k j}$ tenemos $\frac{w_{i j}}{w_{i k}}+\frac{w_{i j}}{w_{k j}}=2+\frac{w_{k j}}{w_{i k}}+\frac{w_{i k}}{w_{k j}}$, entonces

$$
\dot{b}_{i j}=\frac{1}{\pi} \sum_{k \neq i, j} \Gamma_{k} \operatorname{Im}\left(\frac{w_{k j}}{w_{i k}}+\frac{w_{i k}}{w_{k j}}\right)
$$

de la identidad 5.2 y notando que $A_{i k j}=\operatorname{Im}\left(\frac{\bar{w}_{i k} w_{k j}}{2}\right)$ se obtiene lo deseado.

\subsection{El cono de las formas}

Vamos a llamar espacio de las formas al subconjunto de $\mathbb{R}^{3}$ que describe un triángulo, usando como coordenadas las distancias cuadradas entre los vértices (es decir, ocupando la función de Herón), obteniendo ecuaciones donde no importa la orientación ó posición del triángulo en el plano. Un análisis geométrico basado en la condición de Herón nos dará como resultado que el espacio de las formas es un cono.

Como sabemos, dado un triángulo $\triangle A B C$ con longitudes $a, b, c$ respectivamente podemos calcular su área $A$ mediante la fórmula de Herón, la cuál viene dada por $A=\sqrt{s(s-a)(s-b)(s-c)}$, donde $s$ es el semiperímetro del triángulo, i.e. $s=(a+b+c) / 2$.

La fórmula de Herón también puede ser escrita como:

$$
\begin{aligned}
A & =\frac{1}{4} \sqrt{(a+b+c)(-a+b+c)(a-b+c)(a+b-c)} \\
A & =\frac{1}{4} \sqrt{2\left(a^{2} b^{2}+a^{2} c^{2}+b^{2} c^{2}\right)-\left(a^{4}+b^{4}+c^{4}\right)} \\
A & =\frac{1}{4} \sqrt{\left(a^{2}+b^{2}+c^{2}\right)^{2}-2\left(a^{4}+b^{4}+c^{4}\right)}
\end{aligned}
$$


La forma que a nosotros nos interesa es la 5.5, ya que es la que ocuparemos para definir el área del triángulo determinada por los cuadrados de las distancias. El conjunto $\mathcal{F}$ de triadas $\left(b_{1}, b_{2}, b_{3}\right)$ que corresponden a los cuadrados de los lados de un triángulo queda definido por

$$
\mathcal{F}=\left\{\left(b_{1}, b_{2}, b_{3}\right) \in \mathbb{R}^{3} \mid A^{\prime} \geq 0 \text { y } b_{i} \geq 0, i=1,2,3\right\}
$$

donde $A^{\prime}=\left(b_{1}+b_{2}+b_{3}\right)^{2}-2\left(b_{1}^{2}+b_{2}^{2}+b_{3}^{2}\right)$ viene dada por la condición de Herón para el área.

Consideremos la intersección del plano

$$
P_{k}=\left\{\left(b_{1}, b_{2}, b_{3}\right) \in \mathbb{R}^{3} \mid b_{1}+b_{2}+b_{3}=k\right\}
$$

con el conjunto $\mathcal{F}$. Cualquier punto $\left(b_{1}, b_{2}, b_{3}\right) \in \mathbb{R}^{3}$ se puede escribir como $\left(b_{1}, b_{2}, b_{3}\right)=\lambda\left(\bar{b}_{1}, \bar{b}_{2}, \bar{b}_{3}\right), \lambda \in \mathbb{R}^{+}$, donde $\bar{b}_{1}+\bar{b}_{2}+\bar{b}_{3}=1$. Entonces, denotando $R=\bar{b}_{1}^{2}+\bar{b}_{2}^{2}+\bar{b}_{3}^{2}$, tenemos que

$$
\begin{aligned}
A^{\prime} & =\left(b_{1}+b_{2}+b_{3}\right)^{2}-2\left(b_{1}^{2}+b_{2}^{2}+b_{3}^{2}\right) \\
& =\lambda^{2}\left(\bar{b}_{1}+\bar{b}_{2}+\bar{b}_{3}\right)^{2}-2 \lambda^{2}\left(\bar{b}_{1}^{2}+\bar{b}_{2}^{2}+\bar{b}_{3}^{2}\right) \\
& =\lambda^{2}-2 \lambda^{2} R^{2} \\
& =\lambda^{2}\left(1-2 R^{2}\right) \geq 0
\end{aligned}
$$

sólo si $\lambda=0$ ó $R^{2} \leq \frac{1}{2}$, es decir $R \leq \frac{1}{\sqrt{2}}$. Definamos

$$
D=\left\{\left(\bar{b}_{1}, \bar{b}_{2}, \bar{b}_{3}\right) \in \mathbb{R}^{3} \mid \bar{b}_{1}+\bar{b}_{2}+\bar{b}_{3}=1 \text { y } \bar{b}_{1}^{2}+\bar{b}_{2}^{2}+\bar{b}_{3}^{2} \leq \frac{1}{2}\right\}
$$

Así, $D$ es el disco de radio $1 / \sqrt{6}$ en el plano $P_{1}$ cuyo centro yace en la línea $\mathcal{E}$ que pasa por el origen y es paralela al vector $(1,1,1)$. De hecho, es evidente que para toda $k \in \mathbb{R}^{+}, \mathcal{F} \cap P_{k}$ es un disco en el plano $P_{k}$ con centro sobre $\mathcal{E}$ y el conjunto $\mathcal{F}$ es un cono sólido circular recto generado por el disco $D$ y el eje $\mathcal{E}$.

Podemos pensar a $\mathcal{F}$ (o bien, a $D$ ) como el conjunto de todas las clases de equivalencia de configuraciones de tres vórtices en el plano, donde dos configuraciones son equivalentes si forman triángulos congruentes (o bien, triángulos semejantes) con lados homólogos formados por los mismos vórtices. Es por esta razón que nos referimos a $\mathcal{F}$ como el cono de las formas.

El eje $\mathcal{E}$ del cono corresponde a la familia de triángulos equiláteros, es decir, aquellos para los cuales $b_{1}=b_{2}=b_{3}$. La frontera $\partial \mathcal{F}$ del cono corresponde a $A^{\prime}=0$, es decir, configuraciones colineales. 
El cono de las formas es tangente a los tres planos cartesianos. Cada una de las tres líneas rectas de tangencia corresponde a colisiones binarias. En efecto, consideremos la recta de tangencia sobre el plano $y=0$ y sea $P(x, 0, z)$ un punto sobre dicha línea. Entonces $x+z=1$ y $x^{2}+z^{2}=\frac{1}{2}$, ya que son las condiciones ya conocidas de un punto sobre la frontera del cono con $y=0$. Entonces tenemos

$$
\begin{aligned}
1^{2} & =(x+z)^{2} \\
& =x^{2}+z^{2}+2 x z \\
& =\frac{1}{2}+2 x z
\end{aligned}
$$

por lo que $2 x z=\frac{1}{2}$. Por otra parte, consideremos

$$
\begin{aligned}
(x-z)^{2} & =x^{2}+z^{2}-2 x z \\
& =\frac{1}{2}-\frac{1}{2} \\
x-z & =0 .
\end{aligned}
$$

Por lo tanto $x=z$, es decir, se trata de una colisión binaria. De manera análoga razonamos para los planos cartesianos $x=0$ y $z=0$.

Concluyamos esta sección con un par de observaciones sobre las ecuaciones de movimiento definidas en el cono de las formas $\mathcal{F}$ :

1. Las ecuaciones $(5.1)$ son singulares en $\partial \mathcal{F}$, en el sentido de que ahí el término $A_{i j k}$ no tiene derivada continua, debido a la raíz cuadrada en (5.5). Por lo tanto, el Teorema de Unicidad de Ecuaciones Diferenciales Ordinarias no se aplica en puntos sobre la frontera del cono de las formas. (De hecho, se deduce del análisis que elaboraremos en el capítulo 6 que la solución con una condición inicial genérica sobre $\partial \mathcal{F}$ consiste en una trayectoria que oscila recorriendo, de ida y vuelta, un segmento de curva en el interior de $\mathcal{F}$ cuyos extremos son la condición inicial dada y otro punto sobre $\partial \mathcal{F}$.)

2. Las ecuaciones (5.1) no son hamiltonianas.

Estos dos puntos hacen que la descripción de la dinámica reducida del problema de tres vórtices no sea enteramente satisfactoria usando únicamente los cuadrados de las distancias. En el siguiente capítulo mejoramos esta situación encajando el cono de las formas en un espacio de dimensión cuatro. 


\section{Capítulo 6}

\section{Álgebra y reducción del problema de tres vórtices}

En el presente capítulo consideraremos al área del triángulo formado por los tres vórtices como una variable independiente de las distancias entre ellos. Esto nos permitirá definir una estructura de Poisson $\{$,$\} en \mathbb{R}^{4}$ y encajar en este espacio el cono de las formas que se introdujo en el capítulo anterior.

En este capítulo consideraremos solo el caso en que las tres vorticidades son iguales. Sin pérdida de la generalidad asumiremos que $\Gamma_{1}=\Gamma_{2}=\Gamma_{3}=1$.

\subsection{Inmersión del cono de las formas en $\mathbb{R}^{4}$}

Sea $\mathcal{V}=\mathbb{R}^{4}=$ el espacio de los triángulos caracterizados por los cuadrados de la longitud de sus lados y su área. El espacio $\mathcal{V}$ incluye tanto a los triángulos geométricamente posibles como a los imposibles; un ejemplo de estos últimos siendo cuando se viola la fórmula de Herón. Un punto en $\mathcal{V}$ (es decir, un triángulo ó pseudo-triángulo) se denota $\left(b_{1}, b_{2}, b_{3}, \Delta\right)$. El área $\Delta$ puede ser positiva ó negativa, permitiendo distinguir la orientación del triángulo. Sea $\beta=\left\{e_{1}, e_{2}, e_{3}, e_{4}\right\}$ la base canónica en $\mathcal{V}$ y sea $\beta^{*}=\left\{\bar{b}_{1}, \bar{b}_{2}, \bar{b}_{3}, \bar{\Delta}\right\}$ su base dual.

Siguiendo las ideas introducidas en [6], notamos que el paréntesis de Poisson canónico definido sobre $\mathbb{C}^{3}$ induce una estructura de Poisson en $\mathcal{V}$ mediante

$$
\begin{aligned}
& \left\{\bar{b}_{i}, \bar{b}_{j}\right\}=-8 \bar{\Delta} \\
& \left\{\bar{b}_{i}, \bar{\Delta}\right\}=\bar{b}_{j}-\bar{b}_{k}
\end{aligned}
$$


con $(i, j, k)$ permutación cíclica de $(1,2,3)$.

Como cualquier funcional lineal sobre $\mathcal{V}$ se escribe como combinación lineal de $\left\{\bar{b}_{1}, \bar{b}_{2}, \bar{b}_{3}, \bar{\Delta}\right\}$, las ecuaciones 6.1 y $(6.2)$ definen un paréntesis de Poisson para funcionales lineales sobre $\mathcal{V}$. Utilizando el teorema siguiente, las ecuaciones (6.1) y 6.2) de hecho determinan un paréntesis de Poisson en $\mathcal{V}$.

Teorema 6.1.1. Un paréntesis de Poisson $\{$,$\} sobre un espacio vectorial$ queda completamente determinado cuando se específica como actúa en funcionales lineales.

Demostración. Véase la sección 10.4 de [24].

Las ecuaciones (6.1) y (6.2) le dan a $\mathcal{V}^{*}$ una estructura de álgebra de Lie (debido a que tenemos definido un braquet de Lie).

\subsubsection{Reducción de Poisson}

Sea $\pi: \mathbb{C}^{3} \longrightarrow \mathcal{V}$ la aplicación que manda la configuración de vórtices $\left(z_{1}, z_{2}, z_{3}\right) \mapsto\left(b_{1}, b_{2}, b_{3}, \Delta\right)$ donde $\operatorname{los} b_{i}=\left|z_{j}-z_{k}\right|$ y $\Delta$ es el área orientada del triángulo con vértices en $z_{1}, z_{2}, z_{3}$. Pensemos a $\pi$ como la proyección del espacio total a un espacio reducido (que olvida la orientación del triángulo formado por los vórtices y la localización de su centro de vorticidad).

Es evidente que la aplicación $\pi$ es invariante bajo rotaciones y traslaciones. Además,

Teorema 6.1.2. La proyección $\pi$ es una trasformación de Poisson.

Demostración. Por el teorema 6.1.1, sólo basta probar que $\{f \circ \pi, g \circ \pi\}(\mathbf{z})=$ $\{f, g\} \circ \pi(\mathbf{z})$ para una base de $\mathcal{V}^{*}$. Consideremos el caso en que $f: \mathbb{R}^{4} \longrightarrow \mathbb{R}$ y $g: \mathbb{R}^{4} \longrightarrow \mathbb{R}$, con $f=\bar{b}_{1}$ y $g=\bar{b}_{2}$, entonces por una parte tenemos

$$
\begin{aligned}
\{f, g\}(\pi(\mathbf{z})) & =\left\{\bar{b}_{1}, \bar{b}_{2}\right\}(\pi(\mathbf{z})) \\
& =-8 \bar{\Delta}\left(b_{1}, b_{2}, b_{3}, \Delta\right) \\
& =-8 \Delta(\mathbf{z}) .
\end{aligned}
$$

Ahora, por otra parte tenemos

$$
\begin{aligned}
\{f \circ \pi, g \circ \pi\}(\mathbf{z}) & =\left\{\bar{b}_{1} \circ \pi, \bar{b}_{2} \circ \pi\right\}(\mathbf{z}) \\
& =\left\{\bar{b}_{1}\left(b_{1}, b_{2}, b_{3}, \Delta\right), \bar{b}_{2}\left(b_{1}, b_{2}, b_{3}, \Delta\right)\right\}(\mathbf{z}) \\
& =\left\{b_{1}, b_{2}\right\}(\mathbf{z}) \\
& =-8 \Delta(\mathbf{z})
\end{aligned}
$$


donde en la penúltima igualdad el braquet se considera como el braquet canónico dado por (2.17).

Realizando el mismo procedimiento para $\{f, g\}(\pi(\mathbf{z}))$ con $f=\bar{b}_{2}, \bar{b}_{3}, \bar{\Delta}_{\mathrm{y}}$ $g=\bar{b}_{3}, \bar{b}_{1}, \bar{\Delta}$, obtenemos que

$$
\{f \circ \pi, g \circ \pi\}(\mathbf{z})=\{f, g\} \circ \pi(\mathbf{z})
$$

Sea $\mathcal{V}_{0}$ la imagen de $\pi$. Así, por construcción,

$$
\pi: \mathbb{C}^{3} \longrightarrow \mathcal{V}_{0} \subset \mathcal{V}
$$

es suprayectiva. Por geometría elemental,

$$
\mathcal{V}_{0}=\left\{\left(b_{1}, b_{2}, b_{3}, \Delta\right) \subset \mathbb{R}^{4} \mid H\left(b_{1}, b_{2}, b_{3}, \Delta\right)=0, b_{i}>0\right\},
$$

donde $H\left(b_{1}, b_{2}, b_{3}, \Delta\right)$ es la función de Herón determinada a partir de la ecuación (5.5):

$$
H\left(b_{1}, b_{2}, b_{3}, \Delta\right)=16 \Delta^{2}+b_{1}^{2}+b_{2}^{2}+b_{3}^{2}-2\left(b_{1} b_{2}+b_{3} b_{1}+b_{2} b_{3}\right) .
$$

Notemos que $H\left(b_{1}, b_{2}, b_{3}, \Delta\right)$ es homogénea de grado dos, por lo que $\mathcal{V}_{0}$ es un cono en $\mathbb{R}^{4}$, ó bien un hipercono.

Es interesante notar que la condición $H\left(b_{1}, b_{2}, b_{3}, \Delta\right)=0$ es equivalente a

$$
\delta^{2}=-2 X^{2}+Y^{2}
$$

donde

$$
\begin{aligned}
\delta & =4 \Delta \\
X & =\sqrt{\frac{2}{3}\left(b_{1}^{2}+b_{2}^{2}+b_{3}^{2}-b_{1} b_{2}-b_{2} b_{3}-b_{3} b_{1}\right)}, \\
Y & =\left(b_{1}+b_{2}+b_{3}\right) / \sqrt{3} .
\end{aligned}
$$

Las variables $X$ y $Y$ tienen la siguiente interpretación geométrica. Dado $P=\left(b_{1}, b_{2}, b_{3}\right) \in \mathbb{R}^{3}$,

1. La variable $X$ representa la distancia de $P$ al eje $\mathcal{E}$ del cono de las formas discutido en la sección 5.3, es decir, la distancia de $P$ a la línea que pasa por el origen en la dirección de $(1,1,1)$. 


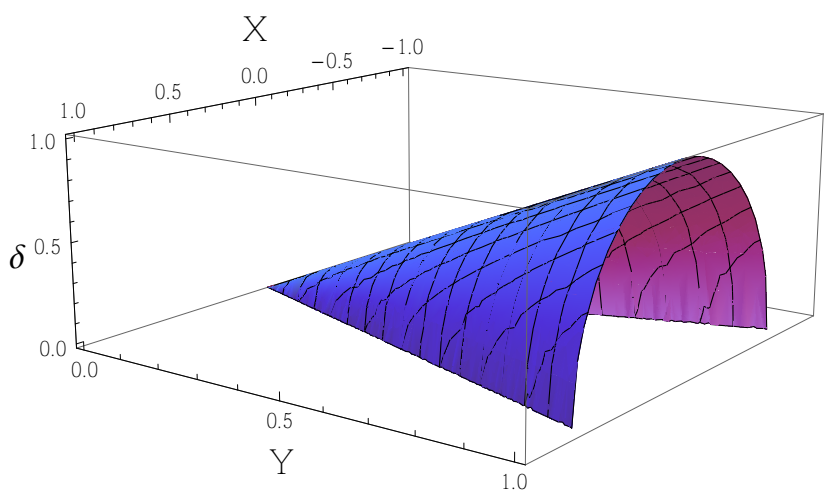

Figura 6.1: Parte superior del cono $\delta^{2}=-2 X^{2}+Y^{2}$. Representa la imagen, bajo $\pi: \mathbb{C}^{3} \longrightarrow \mathcal{V}_{0}$, de los triángulos positivamente orientados.

2. La variable $Y$ representa la distancia de $P$ al plano $b_{1}+b_{2}+b_{3}=0$.

Por lo anterior, $\mathcal{V}_{0}$ también se puede caracterizar como

$$
\mathcal{V}_{0}=\left\{\left(b_{1}, b_{2}, b_{3}, \Delta\right) \subset \mathcal{V} \mid \delta^{2}=-2 X^{2}+Y^{2}, Y>0\right\}
$$

donde $\delta, X$ y $Y$ están definidas por (6.4)-(6.6).

Sea $f_{3} \in \mathbb{R}^{3}$ el vector unitario en la dirección de $(1,1,1)$ y sea $\left(f_{1}, f_{2}, f_{3}\right)$ una base ortonormal de $\mathbb{R}^{3}$. Así, el hipercono definido por la ecuación (6.3) también queda caracterizado por la ecuación

$$
\delta^{2}=-2\left(X_{1}^{2}+X_{2}^{2}\right)+Y^{2}
$$

donde $\left(X_{1}, X_{2}, Y\right)$ son las coordenadas, con respecto a la base $\left(f_{1}, f_{2}, f_{3}\right)$, del punto que en la base estándar tiene coordenadas $\left(b_{1}, b_{2}, b_{3}\right)$.

Sea $\Delta\left(b_{1}, b_{2}, b_{3}\right)$ definido implícitamente mediante la condición $H\left(b_{1}, b_{2}, b_{3}, \Delta\right)=$ 0 . Entonces la aplicación

$$
\left(b_{1}, b_{2}, b_{3}\right) \mapsto\left(b_{1}, b_{2}, b_{3}, \Delta\left(b_{1}, b_{2}, b_{3}\right)\right)
$$

define una inmersión del cono de las formas descrito en el capítulo 5 en $\mathcal{V} \cong \mathbb{R}^{4}$. La imagen de esta inmersión es la parte superior ó inferior del cono dependiendo del signo que escojamos para $\Delta$ : positivo ó negativo según la orientación que elijamos para el triángulo formado por los tres vórtices.

La figura 6.1 representa la imagen, en el espacio reducido, de los triángulos positivamente orientados. Junto con la parte inferior del cono, la figura 6.1 representa la doble inmersión del cono de las formas en $\mathbb{R}^{4}$. 


\subsection{Isomorfismo entre $\mathcal{V}$ y $\mathfrak{u}(2)^{*}$}

En esta sección establecemos que el espacio $\mathcal{V}$ es isomorfo, como variedad de Poisson, al dual del álgebra de Lie del grupo unitario $U(2)$.

Veamos primero que existe un isomorfismo entre $\mathcal{V}^{*}$ y $\mathfrak{u}(2)$. Para ello consideremos $\sigma=\left\{\sigma_{0}, \sigma_{1}, \sigma_{2}, \sigma_{3}\right\}$ la base de $\mathfrak{u}(2)$, definida como $\sigma_{0}=i \varsigma_{0}$, $\sigma_{1}=i \varsigma_{1}, \sigma_{2}=i \varsigma_{2}$ y $\sigma_{3}=i \varsigma_{3}$, donde

$$
\varsigma_{0}:=\left(\begin{array}{ll}
1 & 0 \\
0 & 1
\end{array}\right) \quad(\text { la matriz identidad })
$$

$\mathrm{y}$

$$
\varsigma_{1}:=\left(\begin{array}{cc}
0 & 1 \\
1 & 0
\end{array}\right), \quad \varsigma_{2}:=\left(\begin{array}{cc}
0 & -i \\
i & 0
\end{array}\right), \quad \varsigma_{3}:=\left(\begin{array}{cc}
1 & 0 \\
0 & -1
\end{array}\right)
$$

son las matrices de Pauli. Así,

$$
\sigma_{0}=\left(\begin{array}{ll}
i & 0 \\
0 & i
\end{array}\right), \quad \sigma_{1}=\left(\begin{array}{ll}
0 & i \\
i & 0
\end{array}\right), \quad \sigma_{2}=\left(\begin{array}{cc}
0 & 1 \\
-1 & 0
\end{array}\right), \quad \sigma_{3}=\left(\begin{array}{cc}
i & 0 \\
0 & -i
\end{array}\right) .
$$

Definición 6.2.1. Sea ${ }^{\wedge}: \mathbb{R}^{3} \longrightarrow \mathcal{V}_{1}^{*}$ definido como $(x, y, z)^{\wedge}=x \bar{b}_{1}+y \bar{b}_{2}+$ $z \bar{b}_{3}$, donde $\mathcal{V}_{1}^{*} \subset \mathcal{V}, \mathcal{V}_{1}^{*}=\operatorname{span}\left\{\bar{b}_{1}, \bar{b}_{2}, \bar{b}_{3}\right\}$

Primero veamos que para las matrices descritas anteriormente se verifican las propiedades del conmutador $[A, B]=A B-B A$, obteniendo

$$
\begin{aligned}
& {\left[\sigma_{1}, \sigma_{2}\right]=-2 \sigma_{3}} \\
& {\left[\sigma_{2}, \sigma_{3}\right]=-2 \sigma_{1}} \\
& {\left[\sigma_{3}, \sigma_{1}\right]=-2 \sigma_{2}}
\end{aligned}
$$

mientras que

$$
\begin{aligned}
& {\left[\sigma_{0}, \sigma_{1}\right]=\left(\begin{array}{ll}
0 & 0 \\
0 & 0
\end{array}\right)} \\
& {\left[\sigma_{0}, \sigma_{2}\right]=\left(\begin{array}{ll}
0 & 0 \\
0 & 0
\end{array}\right)} \\
& {\left[\sigma_{0}, \sigma_{3}\right]=\left(\begin{array}{ll}
0 & 0 \\
0 & 0
\end{array}\right)}
\end{aligned}
$$


Por otro lado, en $\mathcal{V}^{*}$ calculamos:

$$
\begin{aligned}
\left\{\bar{b}_{1}+\bar{b}_{2}+\bar{b}_{3}, \bar{b}_{1}\right\} & =\left\{\bar{b}_{2}, \bar{b}_{1}\right\}+\left\{\bar{b}_{1}, \bar{b}_{3}\right\} \\
& =8 \bar{\Delta}-8 \bar{\Delta} \\
& =0 \\
\left\{\bar{b}_{1}+\bar{b}_{2}+\bar{b}_{3}, \bar{\Delta}\right\} & =\left\{\bar{b}_{1}, \bar{\Delta}\right\}+\left\{\bar{b}_{2}, \bar{\Delta}\right\}+\left\{\bar{b}_{1}, \bar{\Delta}\right\} \\
= & \left(\bar{b}_{2}-\bar{b}_{3}\right)+\left(\bar{b}_{3}-\bar{b}_{1}\right)+\left(\bar{b}_{1}-\bar{b}_{2}\right) \\
= & 0
\end{aligned}
$$

Lo mismo si lo calculamos para $\left\{\bar{b}_{1}+\bar{b}_{2}+\bar{b}_{3}, \bar{b}_{2}\right\}=0$ y $\left\{\bar{b}_{1}+\bar{b}_{2}+\bar{b}_{3}, \bar{b}_{3}\right\}=0$, de donde $(1,1,1)^{\wedge} \in \mathcal{Z}\left(\mathcal{V}^{*}\right)$ (el centro de $\left.\mathcal{V}^{*}\right)$.

Demostramos a continuación que de hecho $\mathcal{Z}\left(\mathcal{V}^{*}\right)$ es el subespacio generado por $(1,1,1)^{\wedge}$.

Sea $\mathbf{u}=\bar{b}_{1}+\bar{b}_{2}+\bar{b}_{3}$, supongamos que $a_{0} \mathbf{u}+a_{1} \bar{b}_{1}+a_{2} \bar{b}_{2}+a_{3} \bar{\Delta} \in \mathcal{Z}\left(\mathcal{V}^{*}\right)$, i.e. $\left\{a_{0} \mathbf{u}+a_{1} \bar{b}_{1}+a_{2} \bar{b}_{2}+a_{3} \bar{\Delta}, Y\right\}=0 \forall Y \in \mathcal{V}^{*}$, de donde tenemos que

$$
\begin{aligned}
& \left\{a_{1} \bar{b}_{1}+a_{2} \bar{b}_{2}, \bar{\Delta}\right\}=0 \\
& \left\{a_{1} \bar{b}_{1}+a_{3} \bar{\Delta}, \bar{b}_{2}\right\}=0 \\
& \left\{a_{2} \bar{b}_{2}+a_{3} \bar{\Delta}, \bar{b}_{1}\right\}=0
\end{aligned}
$$

entonces

$$
\begin{array}{r}
a_{1}\left(\bar{b}_{2}-\bar{b}_{3}\right)+a_{2}\left(\bar{b}_{3}-\bar{b}_{1}\right)=0 \\
-8 a_{1} \bar{\Delta}-a_{3}\left(\bar{b}_{3}-\bar{b}_{1}\right)=0 \\
-8 a_{2} \bar{\Delta}-a_{3}\left(\bar{b}_{2}-\bar{b}_{3}\right)=0 .
\end{array}
$$

Rescribiendo la primera ecuación

$$
-\bar{b}_{1} a_{2}+\bar{b}_{2} a_{1}+\bar{b}_{3}\left(a_{2}-a_{1}\right)=0
$$

ya que $\operatorname{los} \bar{b}_{i} \neq 0$, entonces $a_{1}=a_{2}=0$. Tomando la tercera ecuación y conociendo que $a_{2}=0$, tenemos directamente

$$
-a_{3}\left(\bar{b}_{2}-\bar{b}_{3}\right)=0
$$

Por tanto tenemos que $a_{1}=a_{2}=a_{3}=0$, lo mismo alternado con $\bar{b}_{3}, \bar{b}_{1}, \bar{\Delta}$ que son los faltantes.

Lo anterior prueba el siguiente resultado: 
Proposición 6.2.2. El centro $\mathcal{Z}\left(\mathcal{V}^{*}\right)$ es el subespacio generado por $(1,1,1)^{\wedge}$.

Ahora, nuestra labor será encontrar una base $\tilde{\sigma}=\left\{\tilde{\sigma}_{0}, \tilde{\sigma}_{1}, \tilde{\sigma}_{2}, \tilde{\sigma}_{3}\right\}$ de $\mathcal{V}^{*}$ tal que $\left\{\tilde{\sigma}_{i}, \tilde{\sigma}_{j}\right\}$ satisfagan las mismas relaciones de conmutación que las matrices de Pauli que generan $\mathfrak{u}(2)$. Para ello necesitaremos las siguientes proposiciones.

Proposición 6.2.3. $\left[x^{\wedge}, \bar{\Delta}\right]=(A x)^{\wedge}$ para todo $x \in \mathbb{R}^{3}$ donde $A=2\left(\begin{array}{ccc}0 & -1 & 1 \\ 1 & 0 & -1 \\ -1 & 1 & 0\end{array}\right)$

Demostración. Por una parte tenemos

$$
\begin{aligned}
{\left[x^{\wedge}, \bar{\Delta}\right] } & =\left[x_{1} \bar{b}_{1}+x_{2} \bar{b}_{2}+\bar{b}_{3}, \bar{\Delta}\right] \\
& =\left[x_{1} \bar{b}_{1}, \bar{\Delta}\right]+\left[x_{2} \bar{b}_{2}, \bar{\Delta}\right]+\left[x_{3} \bar{b}_{3}, \bar{\Delta}\right] \\
& =2 x_{1}\left(\bar{b}_{2}-\bar{b}_{3}\right)+2 x_{2}\left(\bar{b}_{3}-\bar{b}_{1}\right)+2 x_{3}\left(\bar{b}_{1}-\bar{b}_{2}\right) \\
& =2 \bar{b}_{1}\left(x_{3}-x_{2}\right)+2 \bar{b}_{2}\left(x_{1}-x_{3}\right)+2 \bar{b}_{3}\left(x_{2}-x_{1}\right) \\
& =2\left(x_{2}-x_{3}, x_{3}-x_{1}, x_{1}-x_{2}\right)\left(\begin{array}{c}
\bar{b}_{1} \\
\bar{b}_{2} \\
\bar{b}_{3}
\end{array}\right) \\
& =2\left(x_{1}, x_{2}, x_{3}\right)\left(\begin{array}{ccc}
0 & -1 & 1 \\
1 & 0 & -1 \\
-1 & 1 & 0
\end{array}\right)\left(\begin{array}{c}
\bar{b}_{1} \\
\bar{b}_{2} \\
\bar{b}_{3}
\end{array}\right) \\
& =\left(\begin{array}{ccc}
A x)^{\wedge} & \\
0 & -1 & 1 \\
1 & 0 & -1 \\
-1 & 1 & 0
\end{array}\right) \text { se tiene lo deseado. }
\end{aligned}
$$

Proposición 6.2.4. Sea $x \in \mathbb{R}^{3}$ con $x_{1}+x_{2}+x_{3}=0$, entonces

$$
\begin{aligned}
{\left[\left[x^{\wedge}, \bar{\Delta}\right], \bar{\Delta}\right] } & =\left(A^{2} x\right)^{\wedge} \\
& =-3 x^{\wedge}
\end{aligned}
$$

Demostración. Tomemos por una parte

$$
\begin{aligned}
{\left[\left[x^{\wedge}, \bar{\Delta}\right], \bar{\Delta}\right] } & =\left[2 \bar{b}_{1}\left(x_{3}-x_{2}\right)+\bar{b}_{2}\left(x_{1}-x_{3}\right)+2 \bar{b}_{3}\left(x_{2}-x_{1}\right), \bar{\Delta}\right] \\
& =2\left(x_{3}-x_{2}\right)\left[\bar{b}_{1}, \bar{\Delta}\right]+2\left(x_{1}-x_{3}\right)\left[\bar{b}_{2}, \bar{b}_{2}, \bar{\Delta}\right]+2\left(x_{2}-x_{1}\right)\left[\bar{b}_{3}, \bar{\Delta}\right] \\
& =2\left(x_{3}-x_{2}\right)\left(\bar{b}_{2}-\bar{b}_{3}\right)+2\left(x_{1}-x_{3}\right)\left(\bar{b}_{3}-\bar{b}_{1}\right)+2\left(x_{2}-x_{1}\right)\left(\bar{b}_{1}-\bar{b}_{2}\right) \\
& =2 \bar{b}_{1}\left(x_{2}+x_{3}-2 x_{1}\right)+2 \bar{b}_{2}\left(x_{3}+x_{1}-2 x_{2}\right)+2 \bar{b}_{3}\left(x_{1}+x_{2}-2 x_{3}\right)
\end{aligned}
$$


Por otra parte

$$
A^{2}=\left(\begin{array}{ccc}
-2 & 1 & 1 \\
1 & -2 & 1 \\
1 & 1 & -2
\end{array}\right)
$$

entonces, tenemos que

$$
\begin{aligned}
(A x)^{\wedge} & =\left(x_{1}, x_{2}, x_{3}\right)\left(\begin{array}{ccc}
-2 & 1 & 1 \\
1 & -2 & 1 \\
1 & 1 & -2
\end{array}\right)\left(\begin{array}{l}
\bar{b}_{1} \\
\bar{b}_{2} \\
\bar{b}_{3}
\end{array}\right) \\
& =2 \bar{b}_{1}\left(x_{2}+x_{3}-2 x_{1}\right)+2 \bar{b}_{2}\left(x_{3}+x_{1}-2 x_{2}\right)+2 \bar{b}_{3}\left(x_{1}+x_{2}-2 x_{3}\right)
\end{aligned}
$$

Proposición 6.2.5. Sea $x \in \mathbb{R}^{3}$ con $x_{1}+x_{2}+x_{3}=0$ y $\|x\|^{2}=1$, entonces

$$
\left[x^{\wedge},\left[x^{\wedge}, \bar{\Delta}\right]\right]=-24 \bar{\Delta}
$$

Demostración.

$$
\begin{aligned}
{\left[x^{\wedge},\left[x^{\wedge}, \bar{\Delta}\right]\right] } & =\left[x^{\wedge},-2\left(x_{2}-x_{3}\right) \bar{b}_{1}\right]+\left[x^{\wedge},-2\left(x_{3}-x_{1}\right) \bar{b}_{2}\right]+\left[x^{\wedge},-2\left(x_{1}-x_{2}\right) \bar{b}_{3}\right] \\
& =-16 \bar{\Delta}\left[-\frac{1}{2}\left(x_{1}^{2}+x_{2}^{2}+x_{3}^{2}+2\left(x_{1} x_{2}+x_{2} x_{3}+x_{3} x_{1}\right)\right)+\frac{3}{2}\left(x_{1}^{2}+x_{2}^{2}+x_{3}^{2}\right)\right] \\
& =-16 \bar{\Delta}\left[-\frac{1}{2}\left(x_{1}+x_{2}+x_{3}\right)^{2}+\frac{3}{2}\left(x_{1}^{2}+x_{2}^{2}+x_{3}^{2}\right)^{2}\right] \\
& =-16\left(\frac{3}{2} \bar{\Delta}\right) \\
& =-24 \bar{\Delta}
\end{aligned}
$$

Consideremos

$$
\begin{aligned}
& \tilde{\sigma}_{1}=\beta x^{\wedge} \\
& \tilde{\sigma}_{2}=-\frac{1}{2}\left[\tilde{\sigma}_{3}, \tilde{\sigma}_{1}\right] \\
& \tilde{\sigma}_{3}=\alpha \frac{\Delta}{}
\end{aligned}
$$

de donde podemos ver lo siguiente

$$
\begin{aligned}
{\left[\tilde{\sigma}_{1}, \tilde{\sigma}_{2}\right] } & =\left[\beta x^{\wedge},-\frac{1}{2}\left[\alpha \bar{\Delta}, \beta x^{\wedge}\right]\right] \\
-2 \alpha \bar{\Delta} & =-12 \alpha \beta \bar{\Delta}
\end{aligned}
$$




$$
\begin{aligned}
{\left[\tilde{\sigma}_{2}, \tilde{\sigma}_{3}\right] } & =\left[-\frac{1}{2}\left[\tilde{\sigma}_{3}, \tilde{\sigma}_{1}\right], \alpha \bar{\Delta}\right] \\
-2 \beta x^{\wedge} & =\frac{\alpha^{2} \beta}{2}\left[\left[x^{\wedge}, \bar{\Delta}\right], \bar{\Delta}\right] \\
-2 \beta x^{\wedge} & =-\frac{3 \alpha^{2} \beta}{2} x^{\wedge}
\end{aligned}
$$

De las ecuaciones 6.11 y 6.12 obtenemos que $\beta=\frac{1}{\sqrt{6}}$ y $\alpha=\frac{2}{\sqrt{3}}$. Conociendo esos valores obtenemos que

$$
\begin{aligned}
\tilde{\sigma}_{2} & =-\frac{1}{2}\left[\alpha \bar{\Delta}, \beta x^{\wedge}\right] \\
& =\frac{1}{3 \sqrt{2}}\left[x^{\wedge}, \bar{\Delta}\right]
\end{aligned}
$$

Con los cálculos anteriores hemos obtenido valores $\alpha$ y $\beta$, tales que, hacen que las relaciones de conmutación de $\tilde{\sigma}_{i}$ coincidan con las $\sigma_{i}$, obteniendo el siguiente resultado:

Proposición 6.2.6. Dado $x=\left(x_{1}, x_{2}, x_{3}\right) \in \mathbb{R}^{3}$ con $x_{1}+x_{2}+x_{3}=0 y$ $\|x\|=1$, sean

$$
\begin{aligned}
& \widetilde{\sigma}_{0}=\bar{b}_{1}+\bar{b}_{2}+\bar{b}_{3} \\
& \widetilde{\sigma}_{1}=\frac{\bar{b}_{1} x_{1}+\bar{b}_{2} x_{2}+\bar{b}_{3} x_{3}}{\sqrt{6}} \\
& \widetilde{\sigma}_{2}=\frac{\bar{b}_{3}\left(x_{1}-x_{2}\right)}{3 \sqrt{2}}+\frac{\bar{b}_{1}\left(x_{2}-x_{3}\right)}{3 \sqrt{2}}+\frac{\bar{b}_{2}\left(x_{3}-x_{1}\right)}{3 \sqrt{2}} \\
& \widetilde{\sigma}_{3}=\frac{2 \bar{\Delta}}{3}
\end{aligned}
$$

entonces la aplicación $\psi: \mathfrak{u}(2) \longrightarrow \mathcal{V}^{*}$ inducida por $\sigma_{i} \mapsto \tilde{\sigma}_{i}, i=0,1,2,3$, es un isomorfismo entre álgebras de Lie.

Demostración. Por construcción, las mismas relaciones de conmutación se satisfacen para las bases $\tilde{\sigma}_{i}$ y $\sigma_{i}$, por lo que tenemos dicho isomorfismo.

Observación 6.2.7. Enfatizamos que la aplicación $\psi: \mathfrak{u}(2) \longrightarrow \mathcal{V}^{*}$ definida en la proposición 6.2.6 depende del parámetro unidimensional

$$
\mathbf{x} \in\left\{\left(x_{1}, x_{2}, x_{3}\right) \in \mathbb{R}^{3} \mid x_{1}+x_{2}+x_{3}=0,\left\|\left(x_{1}+x_{2}+x_{3}\right)\right\|=1\right\} .
$$


Observación 6.2.8. Notemos que, dada una configuración de tres vórtices $\mathbf{z}=\left(z_{1}, z_{2}, z_{3}\right)$, tenemos que $\tilde{\sigma}_{0}(\pi(\mathbf{z}))=\left|z_{1}-z_{2}\right|^{2}+\left|z_{2}-z_{3}\right|^{2}+\left|z_{3}-z_{1}\right|^{2}$. (Aquí $\pi$ es la proyección definida al inicio de la sección 6.1.1.) Por lo tanto, de la expresión (4.4) para el momento angular, obtenemos que

$$
\tilde{\sigma}_{0}=3 I
$$

En otras palabras, identificando $\mathcal{V}$ con $\mathfrak{u}(2)^{*}$ y suponiendo que $\left(a_{0}, a_{1}, a_{2}, a_{3}\right)$ son las coordenadas en $\mathfrak{u}(2)^{*}$ con respecto a la base dual a $\left\{\sigma_{0}, \sigma_{1}, \sigma_{2}, \sigma_{3}\right\}$ (definidas en (6.7)), tenemos que el momento angular es la función

$$
I\left(a_{0}, a_{1}, a_{2}, a_{3}\right)=a_{0} / 3 .
$$

La proposición 6.2.6 nos permite ahora ver que hay un isomorfismo de Poisson $\varphi: \mathcal{V} \longrightarrow \mathfrak{u}(2)^{*}$. Para ello usamos la idea de operador adjunto.

Definición 6.2.9. Si $T: V \longrightarrow W$ es un operador lineal, entonces $T^{*}$, el operador adjunto de $T$, se define como $T^{*}: W^{*} \longrightarrow V^{*}$ dado por

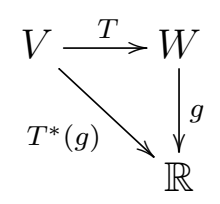

es decir, $T^{*}(g)=g \circ T$. En otras palabras, si $\mathbf{v} \in V,\left\langle T^{*}(g), \mathbf{v}\right\rangle=\langle g, T(\mathbf{v})\rangle$

Lema 6.2.10. Sea $(\mathcal{V},\{\}$,$) un espacio vectorial de Poisson y sea \psi: \mathcal{V}^{*} \longrightarrow \mathfrak{g}$ un isomorfismo entre álgebras de Lie, es decir,

$$
[\psi(\alpha), \psi(\beta)]=\psi(\{\alpha, \beta\})
$$

Sea $\varphi=\left(\psi^{-1}\right)^{*}$, de acuerdo a la definición 6.2.9. En otras palabras, sea $\varphi: \mathcal{V} \longrightarrow \mathfrak{g}^{*}$ donde

$$
\langle\varphi(\mathbf{v}), \xi\rangle=\left\langle\psi^{-1}(\xi), \mathbf{v}\right\rangle
$$

Entonces $\varphi$ es una transformación de Poisson, es decir,

$$
\{f \circ \varphi, h \circ \varphi\}_{\mathbf{v}}=\{f, h\}_{\mathfrak{g}^{*}}
$$


Demostración. Es suficiente considerar el caso $f=\langle\cdot, \xi\rangle, h=\langle\cdot, \eta\rangle$. Entonces $(f \circ \varphi)(\mathbf{v})=\langle\varphi(\mathbf{v}), \xi\rangle=\left\langle\psi^{-1}(\xi), \mathbf{v}\right\rangle$, de donde $f \circ \varphi=\psi^{-1}(\xi)$. Similarmente tenemos $h \circ \varphi=\psi^{-1}(\eta)$, entonces $[\xi, \eta]=\psi(\{f \circ \varphi, h \circ \varphi\})$, pues

$$
\begin{aligned}
\{f \circ \varphi, h \circ \varphi\}(\mathbf{v}) & =\left\langle\psi^{-1}[\xi, \eta], \mathbf{v}\right\rangle \\
& =\langle\varphi(\mathbf{v}),[\eta, \xi]\rangle \\
& =\left\langle\mu,\left[\frac{\delta f}{\delta \mu}, \frac{\delta h}{\delta \mu}\right]\right\rangle
\end{aligned}
$$

$\operatorname{con} \mu=\varphi(\mathbf{v})$.

Sea $\psi: \mathfrak{u}(2) \longrightarrow \mathcal{V}^{*}$ definida por $\psi\left(\sigma_{k}\right)=\tilde{\sigma}_{k}$. Consideremos las respectivas bases de $\mathfrak{u}(2)$ y $\mathcal{V}^{*}, \sigma=\left\{\sigma_{0}, \sigma_{1}, \sigma_{2}, \sigma_{3}\right\}$ y $\beta^{*}=\left\{\bar{b}_{1}, \bar{b}_{2}, \bar{b}_{3}, \bar{\Delta}\right\}$. La matriz de $\psi$ está dada por

$$
[\psi]_{\sigma}^{\beta^{*}}=\left(\begin{array}{cccc}
1 & \frac{x_{1}}{\sqrt{6}} & \frac{x_{3}-x_{2}}{3 \sqrt{2}} & 0 \\
1 & \frac{x_{2}}{\sqrt{6}} & \frac{x_{1}-x_{3}}{3 \sqrt{2}} & 0 \\
1 & \frac{x_{3}}{\sqrt{6}} & \frac{x_{2}-x_{1}}{3 \sqrt{2}} & 0 \\
0 & 0 & 0 & \frac{2}{\sqrt{3}}
\end{array}\right)
$$

Ahora, si a la matriz anterior le aplicamos la transpuesta, obtendremos la siguiente aplicación $\psi^{*}: \mathcal{V} \longrightarrow \mathfrak{u}(2)^{*}$ (ver teorema 2.25 página 121 de [12]), cuya matriz respecto a las bases duales $\sigma^{*}=\left\{\sigma^{0}, \sigma^{1}, \sigma^{2}, \sigma^{3}\right\}$ y $\beta=$ $\left\{e_{1}, e_{2}, e_{3}, e_{4}\right\}$, está dada por

$$
\left[\psi^{*}\right]_{\beta}^{\sigma^{*}}=\left(\begin{array}{cccc}
1 & 1 & 1 & 0 \\
\frac{x_{1}}{\sqrt{6}} & \frac{x_{2}}{\sqrt{6}} & \frac{x_{3}}{\sqrt{6}} & 0 \\
\frac{x_{3}-x_{2}}{3 \sqrt{2}} & \frac{x_{1}-x_{3}}{3 \sqrt{2}} & \frac{x_{2}-x_{1}}{3 \sqrt{2}} & 0 \\
0 & 0 & 0 & \frac{2}{\sqrt{3}}
\end{array}\right)
$$

Por lo tanto, $\varphi \stackrel{\text { def }}{=}\left(\psi^{*}\right)^{-1}: \mathfrak{u}(2)^{*} \longrightarrow \mathcal{V}$ y su matriz con respecto a las bases $\left\{\sigma^{0}, \sigma^{1}, \sigma^{2}, \sigma^{3}\right\}$ y $\left\{e_{1}, e_{2}, e_{3}, e_{4}\right\}$ es

$$
[\varphi]_{\sigma^{*}}^{\beta}=\left(\begin{array}{cccc}
\frac{x_{2}^{2}+x_{3}^{2}-x_{1}\left(x_{2}+x_{3}\right)}{9 \mathrm{D}} & \frac{\sqrt{\frac{2}{3}}\left(2 x_{1}-x_{2}-x_{3}\right)}{3 \mathrm{D}} & \frac{\sqrt{2}\left(x_{3}-x_{2}\right)}{3 \mathrm{D}} & 0 \\
\frac{x_{1}^{2}-x_{2} x_{1}+x_{3}^{2}-x_{2} x_{3}}{9 \mathrm{D}} & -\frac{\sqrt{\frac{2}{3}}\left(x_{1}-2 x_{2}+x_{3}\right)}{3 \mathrm{D}} & \frac{\sqrt{2}\left(x_{1}-x_{3}\right)}{3 \mathrm{D}} & 0 \\
\frac{x_{1}^{2}-x_{3} x_{1}+x_{2}\left(x_{2}-x_{3}\right)}{9 \mathrm{D}} & -\frac{\sqrt{\frac{2}{3}}\left(x_{1}+x_{2}-2 x_{3}\right)}{3 \mathrm{D}} & \frac{\sqrt{2}\left(x_{2}-x_{1}\right)}{3 \mathrm{D}} & 0 \\
0 & 0 & 0 & \frac{\sqrt{3}}{2}
\end{array}\right)
$$

donde $D=\operatorname{det}(\psi)$. 


\subsection{Cantidades conservadas y Casimires}

Sea $H: \mathcal{V} \longrightarrow \mathbb{R}$ la función de Herón, determinada a partir de la ecuación (5.5), a saber:

$$
\begin{aligned}
H\left(b_{1}, b_{2}, b_{3}, \Delta\right) & =(4 \Delta)^{2}-\left(b_{1}+b_{2}+b_{3}\right)^{2}+2\left(b_{1}^{2}+b_{2}^{2}+b_{3}^{2}\right) \\
& =16 \Delta^{2}+b_{1}^{2}+b_{2}^{2}+b_{3}^{2}-2\left(b_{1} b_{2}+b_{3} b_{1}+b_{2} b_{3}\right) .
\end{aligned}
$$

Tenemos el isomorfismo lineal $\varphi: \mathfrak{u}^{*}(2) \longrightarrow \mathcal{V}$. Entonces el pullback de la función de Herón a $\mathfrak{u}^{*}(2)$ está dado por $H \circ \varphi: \mathfrak{u}^{*}(2) \longrightarrow \mathbb{R}$. Un cálculo nos muestra que si $\|\mathbf{x}\|=1$ y $x_{1}+x_{2}+x_{3}=0$ entonces

$$
(H \circ \varphi)\left(a_{0}, a_{1}, a_{2}, a_{3}\right)=\frac{1}{3}\left(-a_{0}^{2}+36\left(a_{1}^{2}+a_{2}^{2}+a_{3}^{2}\right)\right),
$$

donde $\left(a_{0}, a_{1}, a_{2}, a_{3}\right)$ son las coordenadas en $\mathfrak{u}^{*}(2)$ con respecto a la base $\sigma^{*}$.

Observación 6.3.1. Notar que $H \circ \varphi$ es independiente de la elección del parámetro unidimensional $\mathbf{x}$ definido en 6.13 .

Sea $B$ la matriz de la estructura de Poisson definida por 6.1) y 6.2), es decir

$$
B=\left(\begin{array}{cccc}
0 & -8 \bar{\Delta} & 8 \bar{\Delta} & \bar{b}_{2}-\bar{b}_{3} \\
8 \bar{\Delta} & 0 & -8 \bar{\Delta} & \bar{b}_{3}-\bar{b}_{1} \\
-8 \bar{\Delta} & 8 \bar{\Delta} & 0 & \bar{b}_{1}-\bar{b}_{2} \\
\bar{b}_{3}-\bar{b}_{2} & \bar{b}_{1}-\bar{b}_{3} & \bar{b}_{2}-\bar{b}_{1} & 0
\end{array}\right)
$$

de tal forma que $\{f, g\}=(\nabla f)^{T} \cdot B \cdot \nabla h$. Consideremos el vector $\mathbf{u}=$ $(1,1,1,0)$. Calculando $B \cdot \mathbf{u}=0$ tenemos que $\mathbf{u} \in \operatorname{ker} B$. Por otro lado, tomando la función de Herón definida como $H=16 \Delta^{2}+b_{1}^{2}+b_{2}^{2}+b_{3}^{2}-$ $2\left(b_{1} b_{2}+b_{3} b_{1}+b_{2} b_{3}\right)$, y el vector

$$
\nabla H=\left(2\left(b_{1}-b_{2}-b_{3}\right), 2\left(b_{2}-b_{1}-b_{3}\right), 2\left(b_{3}-b_{1}-b_{2}\right), 32 \Delta\right),
$$

observamos que

$$
\begin{aligned}
B \cdot \nabla H= & \left(\begin{array}{c}
-32 \bar{\Delta}\left(\bar{b}_{2}-\bar{b}_{3}\right)+32 \bar{\Delta}\left(\bar{b}_{2}-\bar{b}_{3}\right) \\
-32 \bar{\Delta}\left(\bar{b}_{3}-\bar{b}_{1}\right)+32 \bar{\Delta}\left(\bar{b}_{3}-\bar{b}_{1}\right) \\
-32 \bar{\Delta}\left(\bar{b}_{1}-\bar{b}_{2}\right)+32 \bar{\Delta}\left(\bar{b}_{1}-\bar{b}_{2}\right) \\
2\left(\bar{b}_{3} \bar{b}_{1}-\bar{b}_{2} \bar{b}_{1}+\bar{b}_{1} \bar{b}_{2}-\bar{b}_{3} \bar{b}_{2}+\bar{b}_{2} \bar{b}_{3}-\bar{b}_{1} \bar{b}_{3}\right)
\end{array}\right) \\
= & \left(\begin{array}{l}
0 \\
0 \\
0 \\
0
\end{array}\right)
\end{aligned}
$$


Por lo que concluimos que también $\nabla H \in \operatorname{ker} B$.

Por otro lado, si $\bar{\Delta}=0$ es claro que $\operatorname{dim}(\operatorname{ker} B)=2$; y si $\bar{\Delta} \neq 0$ entonces los dos primeros renglones de $B$ son linealmente independientes, por lo que $\operatorname{dim}(\operatorname{ker} B) \leq 2$. Pero como ya establecimos que $u, \nabla H \in \operatorname{ker} B$ entonces concluimos que $\operatorname{dim}(\operatorname{ker} B)=2$. Por lo tanto

Proposición 6.3.2. La función de Herón $H$ y el momento angular $I=$ $\left(b_{1}+b_{2}+b_{3}\right) / 3$ son Casimires de la estructura de Poisson de $\mathcal{V}$ (ecuaciones (6.1) y (6.2)). Más aún, todo Casimir de esta estructura de Poisson es funcionalmente dependiente de $H$ e $I$.

Como variedad de Poisson, $\mathcal{V}$ está foliada por hojas simplécticas (cf. [24, $\S 10.4])$. Además, toda función Casimir es constante sobre hojas simplécticas (cf. ibid.). Recordemos que $\mathcal{O}_{\mu}$ denota la órbita coadjunta en el dual $\mathfrak{g}^{*}$ de un álgebra de Lie conteniendo a $\mu$. Las órbitas coadjuntas (ó sus componentes conexas) son las hojas simplécticas en $\mathfrak{g}^{*}$ (cf. op. cit., §14.3).

Si $\left(H_{0}, I_{0}\right)$ es un valor regular de la función $\mu \in \mathfrak{u}(2)^{*} \mapsto(H, I)$ entonces el conjunto de nivel determinado por $H=H_{0}, I=I_{0}$ es una superficie suave de dimensión dos; llamémosle $S_{0}$. Las observaciones del párrafo anterior implican que si $\mu \in S_{0}$ entonces $\mathcal{O}_{\mu} \subset S_{0}$. De hecho se verifica que $\mathcal{O}_{\mu}=S_{0}$.

Notemos finalmente que $\operatorname{dim} \mathcal{O}_{\mu}=2$ para todo $\mu \in \mathfrak{u}(2)^{*}$ que corresponda a un estado del sistema de tres vórtices distinto a colisión total. En efecto, un cálculo muestra que en las coordenadas $\left(x_{1}, x_{2}, x_{3}, x_{4}\right)$ que usamos para parametrizar las matrices de $\mathfrak{u}(2)^{*}$ según (2.12), la función de Herón toma la forma

$$
H=35 x_{1}^{2}+144 x_{2}^{2}+144 x_{3}^{2}-74 x_{1} x_{4}+35 x_{4}^{2} .
$$

Por lo tanto $H^{-1}(0) \cap \mathrm{PC}=(0,0,0,0)$, donde $\mathrm{PC}$ es el conjunto de puntos críticos considerado en 2.13 . Es claro que $\mu=(0,0,0,0)$ corresponde a $b_{1}=b_{2}=b_{3}=0$, es decir, colisión total. En este caso $\operatorname{dim} \mathcal{O}_{\mu}=0$. Para cualquier otra $\mu$, la proposición 2.2 .12 implica que $\operatorname{dim} \mathcal{O}_{\mu}=2$.

\subsection{Reducción del problema de tres vórtices como una órbita coadjunta}

De la ecuación (4.3), tenemos que $\Gamma_{\alpha}=\Gamma_{\beta}=1$ (para nuestro caso $N=3$ ), de donde obtenemos la siguiente formulación para la energía,

$$
\mathcal{H}=-\frac{1}{4 \pi} \log \left(b_{1} b_{2} b_{3}\right)
$$




\subsection{Reducción del problema de tres vórtices como una órbita} coadjunta

Deseamos estudiar la dinámica de este hamiltoniano en el espacio $\mathfrak{u}^{*}(2)$, por lo que realizamos la composición $\mathcal{H} \circ \varphi$, donde $\varphi: \mathfrak{u}(2)^{*} \longrightarrow \mathcal{V}$ ha sido definido en la página 84, para obtener

$$
\begin{aligned}
& \mathcal{H}\left(a_{0}, a_{1}, a_{2}, a_{3}\right)=\frac{1}{216\left(x_{1}^{2}+x_{3} x_{1}+x_{3}^{2}\right)^{3}} \cdot \\
& \cdot\left(3 \sqrt{2} a_{2}\left(x_{1}-x_{3}\right)-3 \sqrt{6} a_{1}\left(x_{1}+x_{3}\right)+2 a_{0}\left(x_{1}^{2}+x_{3} x_{1}+x_{3}^{2}\right)\right) \\
& \cdot\left(3 \sqrt{6} a_{1} x_{3}-3 \sqrt{2} a_{2}\left(2 x_{1}+x_{3}\right)+2 a_{0}\left(x_{1}^{2}+x_{3} x_{1}+x_{3}^{2}\right)\right) \\
& \cdot\left(3 \sqrt{6} a_{1} x_{1}+3 \sqrt{2} a_{2}\left(x_{1}+2 x_{3}\right)+2 a_{0}\left(x_{1}^{2}+x_{3} x_{1}+x_{3}^{2}\right)\right)
\end{aligned}
$$

donde los $a_{i}$ son los coeficientes asociados a la base $\sigma^{*}$ de $\mathfrak{u}^{*}(2)$.

Ahora, recordemos que tenemos libertad de elegir cualquier $\left(x_{1}, x_{2}, x_{3}\right) \in$ $\mathbb{R}^{3}$ siempre y cuando satisfaga las condiciones

$$
x_{1}+x_{2}+x_{3}=0 \quad \text { y } \quad\|\mathbf{x}\|=1 .
$$

Buscando la terna $\left(x_{1}, x_{2}, x_{3}\right)$ que nos de la expresión más sencilla para $\mathcal{H}\left(a_{0}, a_{1}, a_{2}, a_{3}\right)$ llegamos a la elección $x_{3}=0$, con la cual el hamiltoniano queda expresadd ${ }^{1}$ por

$$
\mathcal{H}\left(a_{0}, a_{1}, a_{2}, a_{3}\right)=\frac{1}{27}\left(a_{0}+6 a_{2}\right)\left(a_{0}^{2}-6 a_{0} a_{2}+9\left(-3 a_{1}^{2}+a_{2}^{2}\right)\right)
$$

Así pues, para definir el isomorfismo entre $\mathfrak{u}(2)^{*}$ y $\mathcal{V}$, de ahora en adelante tomamos la elección $\left(x_{1}, x_{2}, x_{3}\right)=(-1 / \sqrt{2}, 1 / \sqrt{2}, 0)$. Así, la matriz $[\varphi]_{\sigma^{*}}^{\beta}$ dada en (6.17) que define la transformación $\varphi: \mathfrak{u}(2)^{*} \longrightarrow \mathcal{V}$ se reduce a

$$
[\varphi]_{\sigma^{*}}^{\beta}=\left(\begin{array}{cccc}
\frac{1}{3} & -\sqrt{3} & -1 & 0 \\
\frac{1}{3} & \sqrt{3} & -1 & 0 \\
\frac{1}{3} & 0 & 2 & 0 \\
0 & 0 & 0 & \frac{\sqrt{3}}{2}
\end{array}\right) .
$$

\footnotetext{
${ }^{1}$ El mismo resultado se obtiene si elegimos $x_{1}=0$.
} 
Más aún, de la proposición 6.2.6 obtenemos,

$$
\begin{aligned}
& \tilde{\sigma}_{0}=\bar{b}_{1}+\bar{b}_{2}+\bar{b}_{3} \\
& \tilde{\sigma}_{1}=\frac{\bar{b}_{2}-\bar{b}_{1}}{2 \sqrt{3}} \\
& \tilde{\sigma}_{2}=\frac{\bar{b}_{3}}{3}-\frac{\bar{b}_{1}}{6}-\frac{\bar{b}_{2}}{6} \\
& \tilde{\sigma}_{3}=\frac{2}{\sqrt{3}} \Delta
\end{aligned}
$$

Dado que $a_{0}$ es un casimir, entonces de (2.16) tenemos que

$$
\begin{aligned}
\dot{a}_{0} & =\left\{a_{0}, \mathcal{H}\right\} \\
& =0
\end{aligned}
$$

por tanto $\dot{a}_{0}=0$, es decir $a_{0}$ es constante. Esto es de esperarse, ya que $a_{0}$ es proporcional al momento angular, según vimos en la observación 6.2.8.

Dadas la bases $\sigma=\left\{\sigma_{0}, \sigma_{1}, \sigma_{2}, \sigma_{3}\right\}$ y $\sigma^{*}=\left\{\sigma^{0}, \sigma^{1}, \sigma^{2}, \sigma^{3}\right\}$, sean $a_{i} \in \mathbb{R}$ $i=0,1,2,3$, así que escribimos

$$
a_{0} \sigma^{0}+a_{1} \sigma^{1}+a_{2} \sigma^{2}+a_{3} \sigma^{3}=\left(\begin{array}{cc}
-\frac{i}{2}\left(a_{0}+a_{3}\right) & -\frac{1}{2}\left(a_{1} i+a_{2}\right) \\
-\frac{1}{2}\left(a_{1} i-a_{2}\right) & -\frac{i}{2}\left(a_{0}-a_{3}\right)
\end{array}\right) .
$$

Calculando la traza y determinante de la expresión anterior, tenemos

$$
\begin{aligned}
\operatorname{tr}\left(a_{0} \sigma^{0}+a_{1} \sigma^{1}+a_{2} \sigma^{2}+a_{3} \sigma^{3}\right) & =-i a_{0} \\
\operatorname{det}\left(a_{0} \sigma^{0}+a_{1} \sigma^{1}+a_{2} \sigma^{2}+a_{3} \sigma^{3}\right) & =-\frac{1}{4}\left(-a_{0}^{2}+a_{1}^{2}+a_{2}^{2}+a_{3}^{2}\right)
\end{aligned}
$$

Teniendo en cuenta los casimires $I=a_{0} / 3$ y $H=\frac{1}{3}\left(-a_{0}^{2}+36\left(a_{1}^{2}+a_{2}^{2}+a_{3}^{2}\right)\right)$, tenemos que $I=-\frac{1}{3 i} T$ y $H=12\left(\frac{35}{36} T^{2}+4 D\right)$, donde $T=\operatorname{tr}\left(a_{0} \sigma^{0}+a_{1} \sigma^{1}+\right.$ $\left.a_{2} \sigma^{2}+a_{3} \sigma^{3}\right)$ y $D=\operatorname{det}\left(a_{0} \sigma^{0}+a_{1} \sigma^{1}+a_{2} \sigma^{2}+a_{3} \sigma^{3}\right)$. Como el determinante y la traza son invariantes sobre órbitas coadjuntas (según establecimos en la sección 2.2.7), se sigue que los Casimires $H$ e $I$ son constantes sobre orbitas coadjuntas. Esto era de esperarse, de acuerdo a la discusión al final de la sección 6.3. 


\subsection{Reducción del problema de tres vórtices como una órbita} coadjunta

\subsubsection{Superficies de nivel de las primeras integrales}

Resumamos algunos de los resultados obtenidos hasta ahora contestando las siguientes preguntas:

1. ¿Cómo se representan las superficies de nivel de las integrales primeras (la energía $\mathcal{H}$ y el momento angular $I$ ) de nuestro problema de tres vórtices, y su intersección, en el espacio de configuraciones módulo rotaciones y translaciones?

2. ¿Cómo se representan en el espacio de configuraciones módulo rotaciones?

Hemos definido (ver la sección 6.1.1) la proyección

$$
\begin{aligned}
\pi & : \mathbb{C}^{3} \longrightarrow \mathcal{V} \cong \mathbb{R}^{4} \\
\left(z_{1}, z_{2}, z_{3}\right) & \mapsto\left(b_{1}, b_{2}, b_{3}, \Delta\right)
\end{aligned}
$$

con $b_{i}=\left|z_{j}-z_{k}\right|^{2}$ y $\Delta=\operatorname{Im}\left[\left(\bar{z}_{i}-\bar{z}_{k}\right)\left(z_{k}-z_{j}\right)\right] / 2$. (Es decir, $\Delta$ es el área del triángulo con vértices en $z_{1}, z_{2}, z_{3}$.) También hemos definido el isomorfismo lineal

$$
\varphi: \mathfrak{u}(2)^{*} \longrightarrow \mathcal{V}
$$

cuya matriz $[\varphi]_{\sigma^{*}}^{\beta}$ con respecto a las bases $\sigma^{*}$ y $\beta$ está dada en (6.21). Aquí $\beta$ es la base canónica en $\mathbb{R}^{4}$ y $\sigma^{*}$ es la base dual a $\sigma=\left\{\sigma_{0}, \sigma_{1}, \sigma_{2}, \sigma_{3}\right\}$, la base de $\mathfrak{u}(2)$ construida en términos de las matrices de Pauli y dada explícitamente en (6.7). Tanto $\pi$ como $\varphi$ son transformaciones de Poisson.

De (6.18) tenemos que $\left(\varphi^{-1} \circ \pi\right)\left(\mathbb{C}^{3}\right)$, la imagen en $\mathfrak{u}(2)^{*}$ del espacio de configuraciones de nuestro sistema de tres vórtices, es el cono de dimensión tres

$$
\mathcal{C}_{0}: \quad a_{1}^{2}+a_{2}^{2}+a_{3}^{3}=\left(\frac{a_{0}}{6}\right)^{2}, \quad a_{0} \geq 0,
$$

donde las $a_{k}$ son coordenadas en $\mathfrak{u}(2)^{*}$ con respecto a la base $\sigma^{*}$.

El espacio de configuraciones módulo rotaciones y translaciones queda identificado con el cono $\mathcal{C}_{0}$, en el sentido de que si $\mathbf{z}_{1}$ y $\mathbf{z}_{2}$ son dos configuraciones en $\mathbb{C}^{3}$ entonces $\mathbf{z}_{2}$ se obtiene de $\mathbf{z}_{1}$ mediante una rotación y translación rígidas precisamente cuando $\left(\varphi^{-1} \circ \pi\right)\left(\mathbf{z}_{1}\right)=\left(\varphi^{-1} \circ \pi\right)\left(\mathbf{z}_{2}\right)$.

Ya que la translación de una configuración de tres vórtices queda determinada por la posición de su centro de vorticidad $Z_{0}$, entonces el espacio de configuraciones módulo rotaciones queda identificado con $\mathcal{C}_{0} \times \mathbb{C}$. 


\subsection{Reducción del problema de tres vórtices como una órbita} coadjunta

Ahora bien, de la observación 6.2.8 recordamos que el momento angular viene dado por $I=a_{0} / 3$. Por lo tanto la superficie de nivel $\mathcal{M}_{I}$ en el espacio de configuraciones módulo rotaciones y translaciones, correspondiente a un valor dado $I$ del momento angular, queda identificada con la esfera

$$
\mathcal{M}_{I}: \quad a_{1}^{2}+a_{2}^{2}+a_{3}^{2}=\frac{I^{2}}{4}
$$

de radio $I / 2$ y centro en $(3 I, 0,0,0)$, que yace en el hiperplano $a_{0}=3 I$.

De (6.20) tenemos que la superficie de nivel en $\mathfrak{u}(2)^{*}$ correspondiente a un valor dado $\mathcal{H}$ de la energía es el cilindro de dimensión tres

$$
\tilde{\mathcal{E}}_{\mathcal{H}}: \quad\left(a_{0}+6 a_{2}\right)\left(a_{0}^{2}-27 a_{1}^{2}-6 a_{0} a_{2}+9 a_{2}^{2}\right)=27 \mathcal{H} .
$$

(Lo llamamos "cilindro" debido a que la expresión no depende de la coordenada $a_{3}$.) Por lo tanto, la superficie de nivel $\mathcal{E}_{\mathcal{H}}$ en el espacio de configuraciones módulo rotaciones y translaciones, correspondiente a un valor dado $\mathcal{H}$ de la energía, queda identificado con la intersección

$$
\mathcal{E}_{\mathcal{H}}=\tilde{\mathcal{E}}_{\mathcal{H}} \cap \mathcal{C}_{0} .
$$

Notamos también que la intersección de $\tilde{\mathcal{E}}_{\mathcal{H}}$ con el hiperplano de momento angular constante $a_{0}=3 I$ es el cilindro de dimensión dos

$$
\tilde{\mathcal{E}}_{\mathcal{H}, I}: \quad-\left(2 a_{2}+I\right)\left(3 a_{1}^{2}+2 I a_{2}-a_{2}^{2}-I^{2}\right)=\mathcal{H} .
$$

La figura 6.2 muestra secciones transversales de los cilindros $\tilde{\mathcal{E}}_{\mathcal{H}, I}$ para $I=$ $1 / 3$ y varios valores de $\mathcal{H}$.

Por lo tanto, la curva de nivel $\mathcal{E M}_{\mathcal{H}, I}$ en el espacio de configuraciones módulo rotaciones y translaciones, correspondiente a valores dados $(\mathcal{H}, I)$ de la energía y el momento angular, se obtiene intersecando el cilindro $\tilde{\mathcal{E}}_{\mathcal{H}, I}$ con la esfera $\mathcal{M}_{I}$ :

$$
\mathcal{E} \mathcal{M}_{\mathcal{H}, I}=\tilde{\mathcal{E}}_{\mathcal{H}, I} \cap \mathcal{M}_{I}
$$

La figura 6.3 muestra 2 la esfera $\mathcal{M}_{I}$ correspondiente a la superficie de nivel de momento angular $I=1 / 3$. Las curvas mostradas corresponden a las curvas de nivel $\mathcal{E M}_{\mathcal{H}, I}$ para varios valores de la energía $\mathcal{H}$.

Finalmente, en el espacio de configuraciones módulo rotaciones, las superficies de nivel de $I, \mathcal{H}$ y $(\mathcal{H}, I)$ quedan identificadas, respectivamente, con

$$
\mathcal{M}_{I} \times \mathbb{C}, \quad \mathcal{E}_{\mathcal{H}} \times \mathbb{C}, \quad \mathcal{E M}_{\mathcal{H}, I} \times \mathbb{C} .
$$

\footnotetext{
${ }^{2} \mathrm{El}$ lector puede comparar esta figura con el flujo sobre la órbita coadjunta en el problema del cuerpo rígido libre mencionado en la introducción; ver figura 1.3
} 


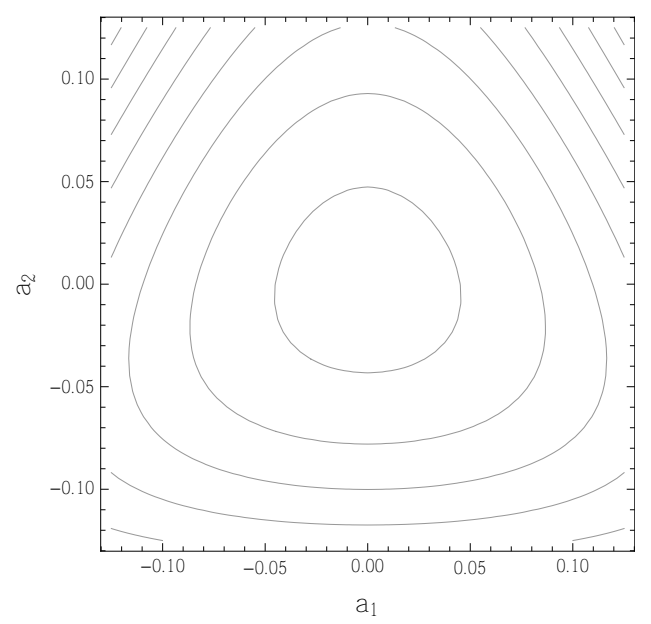

Figura 6.2: Secciones transversales de los cilindros de energía constante.

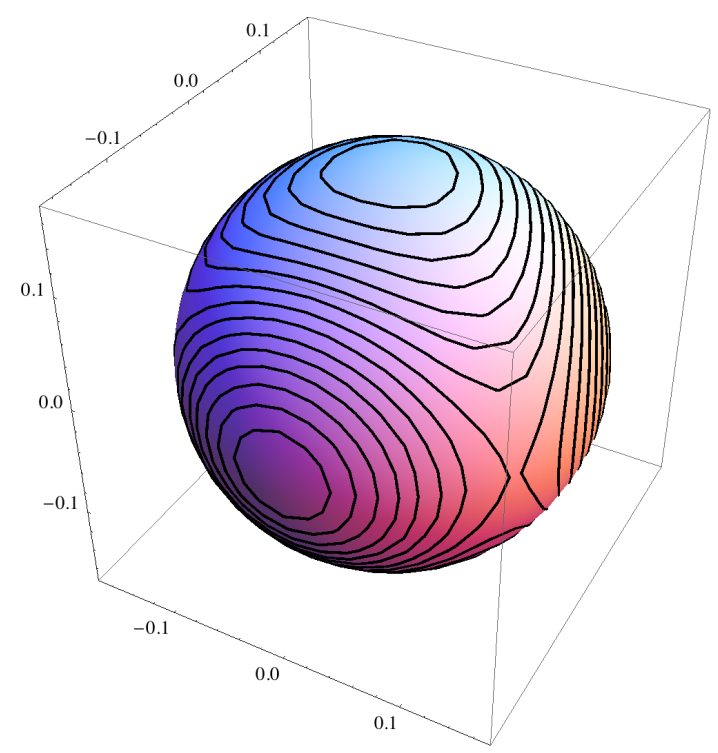

Figura 6.3: Curvas del flujo sobre la órbita coadjunta, correspondiente a $I=1 / 3$. Éstas se obtienen intersecando la esfera de radio $1 / 6$ con los cilindros de energía constante, cuyas secciones transversales se muestran en la figura 6.2 . 


\subsubsection{Estructura simpléctica e interpretación}

Calcularemos a continuación la forma simpléctica definida sobre la órbita coadjunta.

Dada la orbita coadjunta $\mathcal{O}_{\mu}=\left\{\left(a_{0}, a_{1}, a_{2}, a_{3}\right) \mid a_{1}^{2}+a_{2}^{2}+a_{3}^{2}=\left(\frac{a_{0}}{6}\right)^{2}\right\}$, parametrizamos en coordenadas cilíndricas, obteniendo

$$
\begin{aligned}
& a_{1}=\sqrt{\left(\frac{a_{0}}{6}\right)^{2}-z^{2}} \cos \theta \\
& a_{2}=\sqrt{\left(\frac{a_{0}}{6}\right)^{2}-z^{2}} \operatorname{sen} \theta \\
& a_{3}=z
\end{aligned}
$$

Por otra parte, $\operatorname{los} a_{i}, i=0,1,2,3$ los podemos poner en términos de $\theta, z$, es decir

$$
\begin{aligned}
z & =a_{3} \\
\theta & =\arctan \left(\frac{a_{2}}{a_{1}}\right)
\end{aligned}
$$

Ahora, usando la fórmula 2.22 , podemos calcular el braquet $\{f, g\}(\mu)=$ $\left\langle\mu,\left[\frac{\delta f}{\delta \mu}, \frac{\delta g}{\delta \mu}\right]\right\rangle$, donde identificamos a $\frac{\delta f}{\delta \mu}=\nabla f(\mu)$. Para nuestro caso tendre$\operatorname{mos}\{\theta, z\}(\mu)=\left\langle\mu,\left[\frac{\delta \theta}{\delta \mu}, \frac{\delta z}{\delta \mu}\right]\right\rangle$. Primero calculamos

$$
\begin{aligned}
\nabla \theta(\mu) & =\left(0, \frac{a_{2}}{a_{1}^{2}+a_{2}^{2}},-\frac{a_{1}}{a_{1}^{2}+a_{2}^{2}}, 0\right) \\
\nabla z(\mu) & =(0,0,0,1)
\end{aligned}
$$

entonces

$$
\begin{aligned}
\frac{\delta \theta}{\delta \mu} & =\frac{a_{2}}{a_{1}^{2}+a_{2}^{2}} \sigma_{1}-\frac{a_{2}}{a_{1}^{2}+a_{2}^{2}} \sigma_{2} \\
\frac{\delta z}{\delta \mu} & =\sigma_{3}
\end{aligned}
$$

Recordando las propiedades del conmutador $\left[\sigma_{i}, \sigma_{j}\right]=-2 \sigma_{k} i, j, k$ cíclicos, podemos calcular

$$
\begin{aligned}
{\left[\frac{\delta \theta}{\delta \mu}, \frac{\delta z}{\delta \mu}\right] } & =\left[\frac{a_{2}}{a_{1}^{2}+a_{2}^{2}} \sigma_{1}-\frac{a_{2}}{a_{1}^{2}+a_{2}^{2}} \sigma_{2}, \sigma_{3}\right] \\
& =\frac{a_{2}}{a_{1}^{2}+a_{2}^{2}}\left[\sigma_{1}, \sigma_{3}\right]-\frac{a_{1}}{a_{1}^{2}+a_{2}^{2}}\left[\sigma_{2}, \sigma_{3}\right]
\end{aligned}
$$


Entonces, tomando a $\mu=a_{0} \sigma^{0}+a_{1} \sigma^{1}+a_{2} \sigma^{2}+a_{3} \sigma^{3}$ calculamos

$$
\begin{aligned}
\left\langle\mu,\left[\frac{\delta \theta}{\delta \mu}, \frac{\delta z}{\delta \mu}\right]\right\rangle & =\left\langle a_{0} \sigma^{0}+a_{1} \sigma^{1}+a_{2} \sigma^{2}+a_{3} \sigma^{3}, \frac{2}{a_{1}^{2}+a_{2}^{2}}\left(a_{1} \sigma_{1}+a_{2} \sigma_{2}\right)\right\rangle \\
& =2
\end{aligned}
$$

Por lo tanto

$$
B=\left(\begin{array}{cc}
0 & 2 \\
-2 & 0
\end{array}\right) .
$$

Recordemos de la sección 2.2.1, que si $B$ y $J$ son, respectivamente, las matrices representando la estructura de Poisson ${ }^{3}$ y la forma simpléctica sobre una variedad entonces se relacionan mediante

$$
B=\left(J^{-1}\right)^{t} .
$$

De la ecuación (6.24), concluimos que la matriz que representa la forma simpléctica sobre la órbita coadjunta, está dada por

$$
J=\left(\begin{array}{cc}
0 & 1 / 2 \\
-1 / 2 & 0
\end{array}\right)
$$

Por lo tanto de la ecuación 2.5), obtenemos que

$$
\begin{aligned}
X_{\mathcal{H}} & =\left(\begin{array}{cc}
0 & 2 \\
-2 & 0
\end{array}\right)\left[\begin{array}{l}
\partial \mathcal{H} / \partial \theta \\
\partial \mathcal{H} / \partial z
\end{array}\right] \\
& =1 / 2\left[\begin{array}{l}
\partial \mathcal{H} / \partial z \\
\partial \mathcal{H} / \partial \theta
\end{array}\right]
\end{aligned}
$$

es decir

$$
\begin{aligned}
\dot{\theta} & =\frac{1}{2} \frac{\partial \mathcal{H}}{\partial z} \\
\dot{z} & =-\frac{1}{2} \frac{\partial \mathcal{H}}{\partial \theta} .
\end{aligned}
$$

Es decir, llegamos a las ecuaciones de Hamilton clásicas (salvo por el factor $1 / 2$ ).

Usando las ecuaciones de Hamilton podemos calcular la dinámica en las coordenadas cilíndricas $(\theta, z)$. La figura 6.4 muestra cuatro soluciones para 


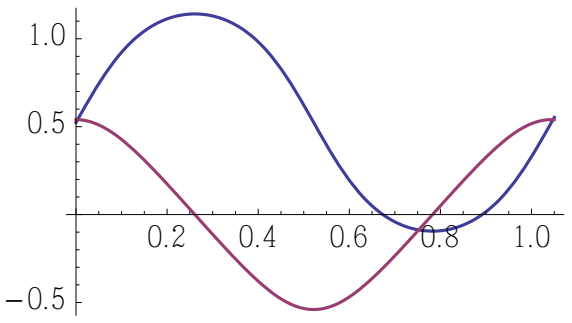

(a)

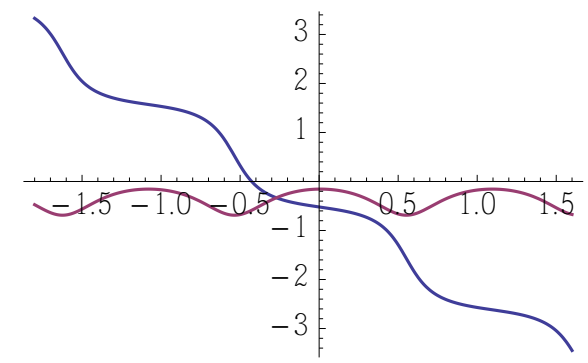

(c)

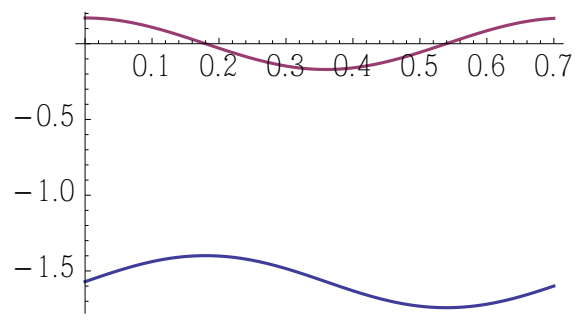

(b)

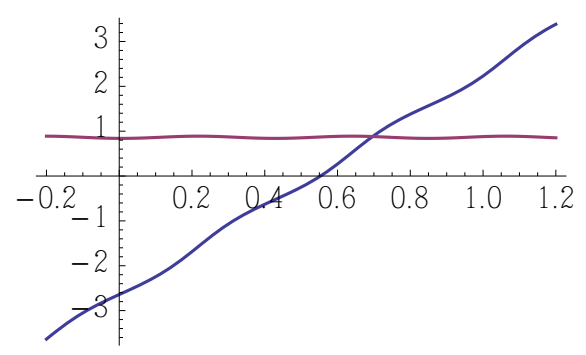

(d)

Figura 6.4: Cuatro soluciones particulares para las ecuaciones de hamilton con $I=2$ y condiciones iniciales $\left(\theta_{0}, z_{0}\right)$ dadas por: (a) $(\pi / 6,0.54)$; (b) $(-\pi / 2,0.17)$; (c) $(-0.5199,-0.1556) ;$ y $(\mathrm{d})(-2.642,0.8396)$. Las curvas en azul representan $\theta(t)$ y las rojas representan $z(t)$. 


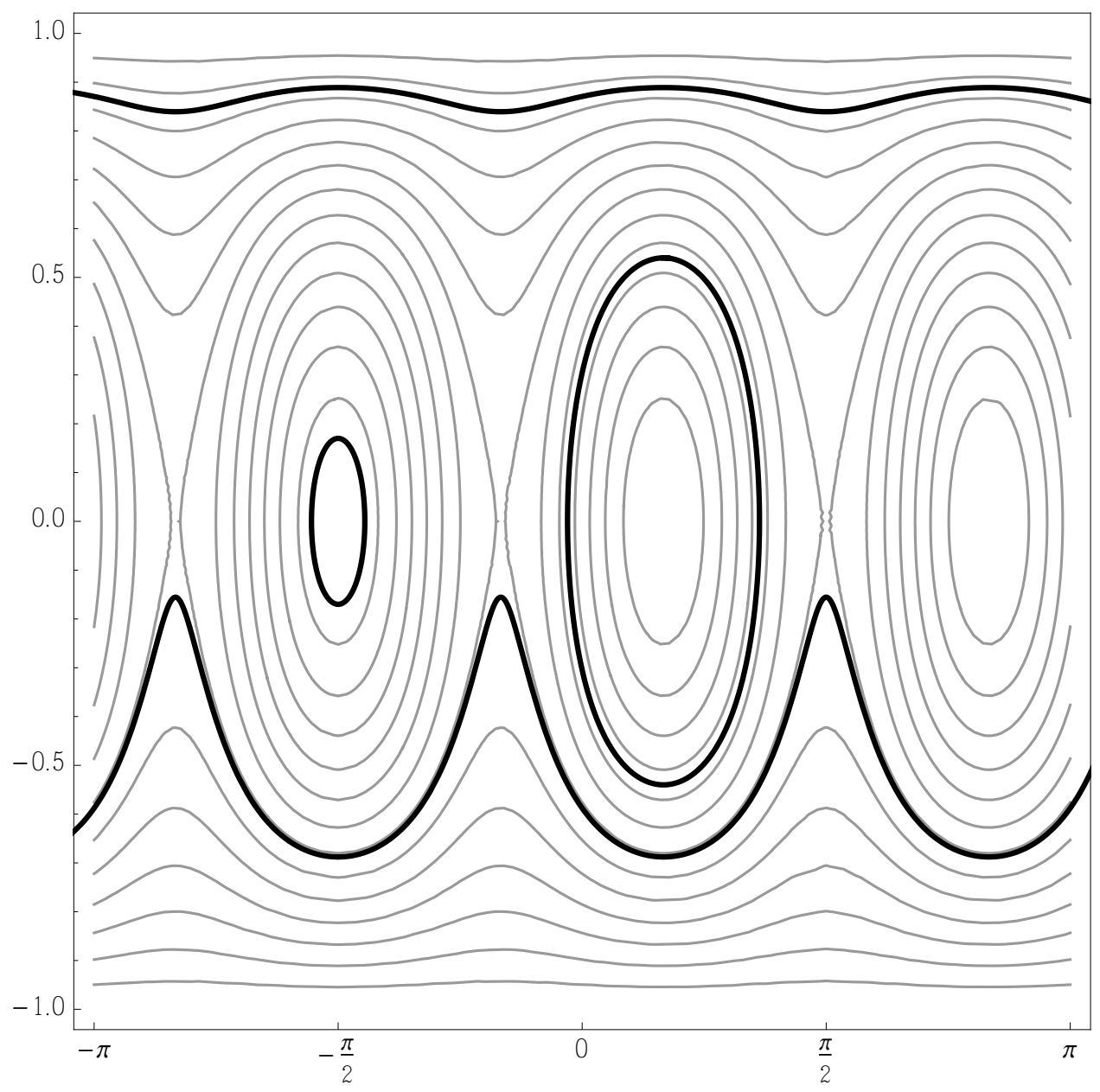

Figura 6.5: Retrato fase junto con trayectorias dinámicas asociadas a las cuatro soluciones particulares mostradas en la figura 6.4 . 


\subsection{Reducción del problema de tres vórtices como una órbita} coadjunta

$I=2$ (por lo que la correspondiente órbita coadjunta tiene radio 1), para cuatro condiciones iniciales distintas. Las correspondientes trayectorias dinámicas se muestran en la figura 6.5 junto con el resto del retrato fase.

La figura 6.5 nos muestra el retrato fase del sistema en coordenadas cilíndricas. (Hemos resaltado las trayectorias $(\theta(t), z(t)$ ) correspondientes a las cuatro soluciones que obtuvimos en la figura 6.4.) Las curvas del retrato fase consisten en las curvas de nivel del hamiltoniano. Observamos cuatro tipos de trayectorias:

1. La separatriz, excluyendo los equilibrios inestables. Las trayectorias de este tipo (seis en total) son las únicas trayectorias no cerradas del sistema, y unen dos equilibrios. Mantienen la misma orientación del triángulo formado por los vórtices.

2. Los tres equilibrios inestables. Son la intersección de la separatriz con el eje $z=0$. Ya que $z$ es proporcional al área del triángulo, estos tres equilibrios corresponden a equilibrios relativos colineales.

3. Las trayectorias que están fuera de la región encerrada por la separatriz, son trayectorias cerradas que mantienen la orientación del triángulo, es decir, en la parte superior corresponde a orientación positiva, mientras que la parte de abajo corresponde a orientación negativa.

4. Los óvalos encerrados por la separatriz. Éstas trayectorias, son cerradas, corresponden a cambios de orientación al cruzar el eje $z=0$, de positiva a negativa. Los tres centros de este tipo de óvalos corresponden a colisiones binarias.

\subsubsection{Colisiones binarias.}

Concluyamos el capítulo verificando que, como se afirmó en el inciso 4 del listado de trayectorias que acabamos de discutir, los centros de los óvalos encerrados por la separatriz corresponden a las tres colisiones binarias. (Notemos que, como se observa en nuestros diagramas de flujo y como se prueba en [16], no hay soluciones dinámicas que terminen en colisión binaria.) A partir de la figura 6.5 podemos estimar que las colisiones binarias ocurren en $\theta=-\pi / 2+n \frac{2 \pi}{3}, n=0,1,2$, y $z=0$.

\footnotetext{
${ }^{3}$ Recordemos que la estructura de Poisson $B$ se define mediante $\{f, g\}=d f \cdot B \cdot \nabla g$ y la matriz simpléctica mediante $\Omega(v, w)=v^{t} \cdot J \cdot w$.
} 


\subsection{Reducción del problema de tres vórtices como una órbita} coadjunta

Dado que la coordenada $z$ corresponde al área del triángulo, es fácil entender que las colisiones binarias yacen sobre la línea $z=0$. Verifiquemos a continuación que las valores de $\theta$ que acabamos de apuntar en efecto corresponden a colisión binaria.

Aplicando ambos lados de las ecuaciones (6.22) al triángulo $T=\left(b_{1}, b_{2}, b_{3}, \Delta\right)=$ $\sum_{k=0}^{3} a_{k} \tilde{\sigma}^{k}$ obtenemos las relaciones entre coeficientes:

$$
\begin{aligned}
& a_{0}=b_{1}+b_{2}+b_{3} \\
& a_{1}=\frac{b_{2}-b_{1}}{2 \sqrt{3}} \\
& a_{2}=\frac{-1}{6}\left(b_{1}+b_{2}-2 b_{3}\right) \\
& a_{3}=\frac{2}{\sqrt{3}} \Delta
\end{aligned}
$$

1. Caso $\theta=-\pi / 2$. Aplicando la transformación a coordenadas cilíndricas 6.23) (tomando en cuenta que $z=0$ ) obtenemos $a_{1}=0, a_{2}=-a_{0} / 6$, por lo que

$$
\begin{aligned}
\frac{b_{2}-b_{1}}{2 \sqrt{3}} & =0 \\
-\frac{1}{6}\left(b_{1}+b_{2}-2 b_{3}\right) & =-\frac{1}{6}\left(b_{1}+b_{2}+b_{3}\right)
\end{aligned}
$$

de donde deducimos que $b_{3}=0$ (colisión binaria entre los vórtices $\Gamma_{1}$ $\left.y \Gamma_{2}\right)$.

2. Caso $\theta=\pi / 6$. De (6.23) obtenemos

$$
a_{1}=\frac{a_{0}}{6} \frac{\sqrt{3}}{2}, \quad a_{2}=\frac{a_{0}}{6} \frac{1}{2}
$$

por lo que

$$
\begin{aligned}
\frac{b_{2}-b_{1}}{2 \sqrt{3}} & =\frac{1}{4 \sqrt{3}}\left(b_{1}+b_{2}+b_{3}\right) \\
\frac{-1}{6}\left(b_{1}+b_{2}-2 b_{3}\right) & =\frac{1}{12}\left(b_{1}+b_{2}+b_{3}\right)
\end{aligned}
$$

de donde deducimos que $b_{1}=0$ (colisión binaria entre los vórtices $\Gamma_{2}$ $\left.y \Gamma_{3}\right)$. 
3. Caso $\theta=5 \pi / 6$. De 6.23 obtenemos

$$
a_{1}=-\frac{a_{0}}{6} \frac{\sqrt{3}}{2}, \quad a_{2}=\frac{a_{0}}{6} \frac{1}{2}
$$

por lo que

$$
\begin{aligned}
\frac{b_{2}-b_{1}}{2 \sqrt{3}} & =-\frac{1}{4 \sqrt{3}}\left(b_{1}+b_{2}+b_{3}\right) \\
\frac{-1}{6}\left(b_{1}+b_{2}-2 b_{3}\right) & =\frac{1}{12}\left(b_{1}+b_{2}+b_{3}\right)
\end{aligned}
$$

de donde deducimos que $b_{2}=0$ (colisión binaria entre los vórtices $\Gamma_{3}$ $\left.y \Gamma_{1}\right)$. 


\section{Capítulo 7}

\section{Conclusiones}

El problema de $N$-vórtices es un problema en Hidrodinámica para el caso que consideramos fluidos ideales, es decir no consideramos viscosidad, por lo cual ocupamos las ecuaciones de Euler. Adicionalmente, consideramos el límite en el que la circulación del fluido está concentrada en puntos discretos. Estudiamos la dinámica en un fluido bidimensional, lo cual tiene la interpretación de considerar filamentos de vorticidad rectilíneos y paralelos. Desde el punto de vista matemático, el problema tiene una estructura Hamiltoniana y una simetría intrínseca debida a la invariancia bajo rotaciones y translaciones.

Después de dar las definiciones de variedades simplécticas y de Poisson, y discutir la dinámica hamiltoniana y de vorticidad, nos dimos a la tarea, en el capítulo 4, de estudiar el problema de $N$-vórtices puntuales en el plano, sus ecuaciones de movimiento y su formulación hamiltoniana. Con miras a hacer uso de la simetría del sistema para reducir el número de grados de libertad, en el capítulo 5 se describieron las ecuaciones de movimiento, para el problema de tres vórtices, en términos de los cuadrados de las distancias. Una desventaja de las ecuaciones así obtenidas fue que perdimos la estructura hamiltoniana. Para recuperar dicha estructura (asumiendo vorticidades iguales), en el capítulo 6 se introdujo el área como un grado de libertad adicional, lo cual permitió convertir a nuestro sistema en un subproblema inmerso en una variedad de Poisson cuatro-dimensional. En este sentido el capítulo 6 es un desarrollo de las ideas introducidas en [6].

De esta manera obtuvimos un problema reducido con una estructura hamiltoniana y adicionalmente una estructura geométrica interesante, a saber, una dinámica en esferas dos-dimensionales que constituyen las hojas 
simplécticas (órbitas coadjuntas) en un espacio de Poisson cuatro-dimensional. Una posible continuación de este trabajo es la implementación de la reducción de Poisson discutida en el capítulo 6 al problema de tres vórtices con vorticidades distintas. En cuyo caso las órbitas coadjuntas no serán necesariamente compactas. Una generalización más podría ser el estudio de la reducción de más de tres vórtices. 


\section{Bibliografía}

[1] V.I. Arnold (1974). The asymptotic Hopf invariant and its applications, (Russian), Sel. Math. Sov. 5, 327 (English).

[2] Aref, H. (1992). Gröbli's solution of the three-vortex problem, Annu. Rev. Fluid Mech. 24,1-20.

[3] C. Bardos (1972). Existence et unicité de la solution de l'équation d'Euler en dimensión deux, J. Math. Anal. App. 40, 769-790.

[4] Batchelor, G. K. (2000). An Introduction to Fluid Dynamics. Cambridge University Press.

[5] J.T. Beale, T. Kato, A. Majda (1984). Remarks on the breakdown of smooth solutions for the three dimensional Euler equations, Comm. Math. Phys. 94, 61-66.

[6] A.V. Borisov \& V. G. Lebedev. Dynamics of three vórtices on a plane and a sphere - II: general compact case. Regular and chaotic dynamics, v.2 (2), 1998.

[7] Martin Celli (2014). Poincaré y el problema de tres vórtices, Miscelanea Matemática 58 103-112.

[8] A.J. Chorin, J. Marsden (1979). A Mathematical Introduction to Fluid Mechanics, Springer-Verlag, New York.

[9] Carmo, Manfredo Perdigao do (1992). Riemannian Geometry, Birkhäuser Boston.

[10] C.R. Doering, J.D. Gibbon (1995). Applied Analysis of the Navier-Stokes Equations, Cambridge University Press, Cambridge. 
[11] Ebin, David G. and Jerrold E. Marsden. Groups of diffeomorphisms on the motion of an incompressible fluid. Ann. of Math., 92, 102-163.

[12] Friedberg, Stephen H., Arnold J. Insel y Lawrence E. Spence (2003). Linear Algebra, Prentice Hall.

[13] R. Grauer, T. Sideris (1991). Numerical computation of three dimensional incompressible ideal fluids with swirl, Phys. Rev. Lett. 67, 35113514 .

[14] R. Grauer, T. Sideris (1995). Finite time singularities in ideal flows with swirl, Physica D 88(2), 116-132.

[15] G. Haller, I. Mezić (1998). Reduction of the three-dimensional, volume preserving flows with simmetry, Nonlinearity 11, 319-339

[16] Hernández-Garduño, A. y E.A. Lacomba (2007). Collisions and Regularization of the 3-Vortex Problem. J. of mathematical fluid mechanics, vol. 9, 75-86.

[17] T. Kato (1967). On classical solutions of the two dimensional nonstationary Euler equations, Arch. Rat. Mech. Anal. 25, 188-200.

[18] T. Kato (1972). Non-stationary flows of viscous and ideal fluids in $\mathbb{R}^{3}$, J. Funct. Anal. 9, 296-305.

[19] R. Kerr (1993). Evidence for a singularity of the three dimensional incompressible Euler equations, Phys. Fluids A 5(7), 1725-1746.

[20] R.H. Kraichnan, D. Montgomery (1980). Two dimensional turbulence, Rep. Prog. Phys. 43(5), 547-619.

[21] P.L. Lions (1996). Mathematical Topics in Fluid Mechanics, Vol. 1 Incompressible Models, Oxford Lecture Series in Mathematics and its Applications, 3, Oxford.

[22] Marsden, J. E. y A. Weinstein, Reduction of symplectic manifolds with symmetry. Rep. Math. Phys., vol. 5 (1974), pp. 121-130.

[23] A.J. Majda, A. Bertrozzi (2001). Vorticity and incompressible Fluid Flow, Cambridge Texts in Applied Mathematics 27, Cambridge Press, Cambridge. 
[24] Marsden, Jerrold E. y Tudor S. Ratiu Introduction to Mechanics and Symmetry, Second Edition, Springer-Verlag.

[25] Marten, Ken, et. al., Ring Bubbles of Dolphins, Scientific American, vol. agosto de 1996.

[26] I. Mezić, S. Wiggins (1994). On the integrability and pertubation of the three-dimensional fluid flows with symmetry, J. Nonlinear Sci. 4, 157-194.

[27] H.K. Moffat (1969). The degree of knottedness of tangled vortex lines, J. Fluid Mech. 35, part 1, 117-129.

[28] H.K. Moffat, A. Tsinobar, eds. (1990). Topological Fluid Mechanics, Cambridge Press, Cambridge.

[29] H.K. Moffat, A. Tsinobar (1992). Helicity in laminar and turbulent flows, Ann. Rev. Fluid Mech. 24, 281-312.

[30] Newton, Paul K. The N-Vortex Problem: Analytical Techniques. Springer (2001).

[31] R. Pelz (1997). Locally self-similar, finite-time collapse in high-simmetry vortex filament model, Phys. Rev. E 55(2), 1617-1626.

[32] Poincaré, H. (1893). Théorie des Tourbillons

[33] G. Ponce (1985). Remarks on a paper by J.T. Beale, T. Kato, and A. Majda, Comm. Math. Phys. 98, 349-353.

[34] A. Pumir, E. Siggia (1992a). Finite time singularities in the axisymmetric three dimensional Euler equations, Phys. Rev. Lett. 68, 1511.

[35] A. Pumir, E. Siggia (1992b). Development of singular solutions to the axisymmetric Euler equations, Phys. Fluids A4, 1472.

[36] P.G. Saffman (1992). Vortex Dynamics, Cambridge University Press, Cambridge.

[37] H. Sánchez Morgado y O. A. Palmas Velasco (2007). Geometría Riemanniana. Publicado por la Facultad de Ciencias, UNAM. 
[38] R. Temam (1975). On the Euler equation of incompressible perfect fluids, J. Funct. Anal. 20, 32-43

[39] R. Temam (1976). Local Existence of $C^{\infty}$ Solutions of the Euler Equations of Incompressible Perfect Fluids, Lecture Notes in Math., 565, Springer-Verlag, New York.

[40] C. Truesdell (1954). The Kinematics of vorticity, Indiana U. Press, Bloomington.

[41] Viatour, Luc. Fotógrafo. http://www.Lucnix.be

[42] W. Wolibner (1933). Un theorém sur l'existence du mouvement plan d'un fluide parfait, homogéne, incompressible, pendent un temps, infinitent long, Math. Z. 37, 698-726.

[43] V.I. Yudovich (1963). Non-stationary flow of an ideal incompressible liquid, Zh. Vych. Mat. 3, 1032-1066. 